\title{
Neuron
}

\section{Genome-wide Analyses Identify KIF5A as a Novel ALS Gene}

\section{Highlights}

- Loss-of-function mutations in KIF5A are a cause of amyotrophic lateral sclerosis

- ALS-associated KIF5A mutations are distinct from HSP and CMT mutations in KIF5A

- Identification of KIF5A highlights the role of cytoskeleton in ALS pathogenesis

\section{Authors}

Aude Nicolas, Kevin P. Kenna, Alan E. Renton, ...,

Christopher E. Shaw, Bryan J. Traynor, John E. Landers

Correspondence

bryan.traynor@nih.gov (B.J.T.), john.landers@umassmed.edu (J.E.L.)

\section{In Brief}

Using a large-scale genome-wide association study and exome sequencing, we identified KIF5A as a novel gene associated with ALS. Our data broaden the phenotype resulting from mutations in KIF5A and highlight the importance of cytoskeletal defects in the pathogenesis of ALS. 


\section{Genome-wide Analyses Identify KIF5A as a Novel ALS Gene}

Aude Nicolas, ${ }^{1,137}$ Kevin P. Kenna, ${ }^{2,137}$ Alan E. Renton, ${ }^{1,3,4,137}$ Nicola Ticozzi, ${ }^{5,6,137}$ Faraz Faghri, ${ }^{7,8,137}$ Ruth Chia, ${ }^{1,137}$ Janice A. Dominov, ${ }^{2}$ Brendan J. Kenna, ${ }^{2}$ Mike A. Nalls, ${ }^{7,9}$ Pamela Keagle, ${ }^{2}$ Alberto M. Rivera, ${ }^{1}$ Wouter van Rheenen, ${ }^{10}$ Natalie A. Murphy, ${ }^{1}$ Joke J.F.A. van Vugt, ${ }^{10}$ Joshua T. Geiger, ${ }^{11}$ Rick A. Van der Spek, ${ }^{10}$ Hannah A. Pliner, ${ }^{1}$ Shankaracharya, ${ }^{2}$ Bradley N. Smith, ${ }^{12}$ Giuseppe Marangi, ${ }^{1,13}$ Simon D. Topp, ${ }^{12}$ Yevgeniya Abramzon, ${ }^{1,14}$ Athina Soragia Gkazi, ${ }^{12}$ John D. Eicher, ${ }^{15}$ Aoife Kenna, ${ }^{2}$ ITALSGEN Consortium, Gabriele Mora, ${ }^{16}$ Andrea Calvo, ${ }^{17}$ Letizia Mazzini, ${ }^{18}$ Nilo Riva, ${ }^{19}$ Jessica Mandrioli, ${ }^{20}$ Claudia Caponnetto, ${ }^{21}$ Stefania Battistini, ${ }^{22}$ Paolo Volanti, ${ }^{16}$ Vincenzo La Bella, ${ }^{23}$ Francesca L. Conforti, ${ }^{24}$ Giuseppe Borghero, ${ }^{25}$ Sonia Messina, ${ }^{26}$ Isabella L. Simone, ${ }^{27}$ Francesca Trojsi, ${ }^{28}$ Fabrizio Salvi, ${ }^{29}$ Francesco O. Logullo, ${ }^{30}$ Sandra D'Alfonso, ${ }^{31}$ Lucia Corrado, ${ }^{31}$ Margherita Capasso, ${ }^{32}$ Luigi Ferrucci, ${ }^{33}$ Genomic Translation for ALS Care (GTAC) Consortium, Cristiane de Araujo Martins Moreno, ${ }^{34}$ Sitharthan Kamalakaran, ${ }^{35}$ David B. Goldstein, ${ }^{35}$ ALS Sequencing Consortium, Aaron D. Gitler, ${ }^{36}$ Tim Harris, ${ }^{37}$ Richard M. Myers, ${ }^{38}$ NYGC ALS Consortium, Hemali Phatnani, ${ }^{39}$ Rajeeva Lochan Musunuri, ${ }^{40}$ Uday Shankar Evani, ${ }^{40}$ Avinash Abhyankar, ${ }^{40}$ Michael C. Zody, ${ }^{40}$ Answer ALS Foundation, Julia Kaye, ${ }^{41}$ Steven Finkbeiner, ${ }^{41,42}$

(Author list continued on next page)

\footnotetext{
${ }^{1}$ Neuromuscular Diseases Research Section, Laboratory of Neurogenetics, National Institute on Aging, NIH, Porter Neuroscience Research Center, Bethesda, MD 20892, USA

2Department of Neurology, University of Massachusetts Medical School, Worcester, MA 01605, USA

${ }^{3}$ Department of Neuroscience, Icahn School of Medicine at Mount Sinai, New York, NY 10029, USA

${ }^{4}$ Ronald M. Loeb Center for Alzheimer's Disease, Icahn School of Medicine at Mount Sinai, New York, NY 10029, USA

${ }^{5}$ Department of Neurology and Laboratory of Neuroscience, IRCCS Istituto Auxologico Italiano, Milan, Italy

${ }^{6}$ Department of Pathophysiology and Transplantation, "Dino Ferrari" Center - Università degli Studi di Milano, Milan 20122, Italy

${ }^{7}$ Molecular Genetics Section, Laboratory of Neurogenetics, National Institute on Aging, NIH, Porter Neuroscience Research Center, Bethesda, MD 20892, USA

${ }^{8}$ Department of Computer Science, University of Illinois at Urbana-Champaign, Urbana, IL, USA

${ }^{9}$ Data Tecnica International, Glen Echo, MD, USA

${ }^{10}$ Department of Neurology, Brain Center Rudolf Magnus, University Medical Center Utrecht, Utrecht, the Netherlands

${ }^{11}$ Neurodegenerative Diseases Research Unit, National Institute of Neurological Disorders and Stroke, NIH, Bethesda, MD 20892, USA

${ }_{12}^{12}$ Maurice Wohl Clinical Neuroscience Institute, Department of Basic and Clinical Neuroscience, King's College London, London SE5 9RS, UK

${ }^{13}$ Institute of Genomic Medicine, Catholic University, Roma, Italy

${ }^{14}$ Sobell Department of Motor Neuroscience and Movement Disorders, University College London, Institute of Neurology, London, UK

${ }^{15}$ Genetics and Pharmacogenomics, MRL, Merck \& Co., Inc., Boston, MA 02115, USA

${ }^{16}$ ALS Center, Salvatore Maugeri Foundation, IRCCS, Mistretta, Messina, Italy

17 "Rita Levi Montalcini” Department of Neuroscience, University of Turin, Turin, Italy

18"Maggiore della Carità" University Hospital, Novara, Italy
}

(Affiliations continued on next page)

\section{SUMMARY}

To identify novel genes associated with ALS, we undertook two lines of investigation. We carried out a genome-wide association study comparing 20,806 ALS cases and 59,804 controls. Independently, we performed a rare variant burden analysis comparing 1,138 index familial ALS cases and 19,494 controls. Through both approaches, we identified kinesin family member $5 A$ (KIF5A) as a novel gene associated with ALS. Interestingly, mutations predominantly in the $\mathrm{N}$-terminal motor domain of KIF5A are causative for two neurodegenerative diseases: hereditary spastic paraplegia (SPG10) and Charcot-MarieTooth type 2 (CMT2). In contrast, ALS-associated mutations are primarily located at the C-terminal cargo-binding tail domain and patients harboring loss-of-function mutations displayed an extended survival relative to typical ALS cases. Taken together, these results broaden the phenotype spectrum resulting from mutations in KIF5A and strengthen the role of cytoskeletal defects in the pathogenesis of ALS.

\section{INTRODUCTION}

Amyotrophic lateral sclerosis (ALS; OMIM: 05400) is a neurodegenerative disorder clinically characterized by rapidly progressive muscle weakness and death due to respiratory failure, typically within 2 to 4 years of symptom onset (van Es et al., 2017). Although ALS is perceived as being rare, approximately 6,000 Americans die annually from the condition 
Stacia K. Wyman, ${ }^{41}$ Alex LeNail, ${ }^{43}$ Leandro Lima, ${ }^{41}$ Ernest Fraenkel, ${ }^{43,44}$ Clive N. Svendsen, ${ }^{45,46}$ Leslie M. Thompson, ${ }^{47,48}$ Jennifer E. Van Eyk, ${ }^{49}$ James D. Berry, ${ }^{50,51}$ Timothy M. Miller, ${ }^{52}$ Stephen J. Kolb, ${ }^{53}$ Merit Cudkowicz, ${ }^{50,51}$ Emily Baxi, ${ }^{54}$ Clinical Research in ALS and Related Disorders for Therapeutic Development (CReATe) Consortium, Michael Benatar, ${ }^{55}$ J. Paul Taylor, ${ }^{56,57}$ Evadnie Rampersaud, ${ }^{58}$ Gang Wu, ${ }^{58}$ Joanne Wuu, ${ }^{55}$ SLAGEN Consortium, Giuseppe Lauria, ${ }^{59}$ Federico Verde, ${ }^{5}$ Isabella Fogh, ${ }^{5,12}$ Cinzia Tiloca, ${ }^{5}$ Giacomo P. Comi, ${ }^{60}$ Gianni Sorarù, ${ }^{61}$ Cristina Cereda, ${ }^{62}$ French ALS Consortium, Philippe Corcia, ${ }^{63}$ Hannu Laaksovirta, ${ }^{64}$ Liisa Myllykangas, ${ }^{65}$ Lilja Jansson, ${ }^{64}$ Miko Valori, ${ }^{64}$ John Ealing, ${ }^{66}$ Hisham Hamdalla, ${ }^{66}$ Sara Rollinson, ${ }^{67}$ Stuart Pickering-Brown, ${ }^{67}$ Richard W. Orrell, ${ }^{68}$ Katie C. Sidle, ${ }^{69}$ Andrea Malaspina, ${ }^{70}$ John Hardy, ${ }^{69}$ Andrew B. Singleton, ${ }^{7}$ Janel O. Johnson, ${ }^{1}$ Sampath Arepalli, ${ }^{71}$ Peter C. Sapp, ${ }^{2}$ Diane McKenna-Yasek, ${ }^{2}$ Meraida Polak, ${ }^{72}$ Seneshaw Asress, ${ }^{72}$ Safa Al-Sarraj, ${ }^{12}$ Andrew King, ${ }^{12}$ Claire Troakes, ${ }^{12}$ Caroline Vance, ${ }^{12}$ Jacqueline de Belleroche, ${ }^{73}$ Frank Baas, ${ }^{74}$ Anneloor L.M.A. ten Asbroek, ${ }^{75}$ José Luis Muñoz-Blanco, ${ }^{76}$ Dena G. Hernandez, ${ }^{71}$ Jinhui Ding, ${ }^{77}$ J. Raphael Gibbs, ${ }^{77}$ Sonja W. Scholz, ${ }^{11,54}$ Mary Kay Floeter, ${ }^{78}$ Roy H. Campbell, ${ }^{8}$

(Author list continued on next page)

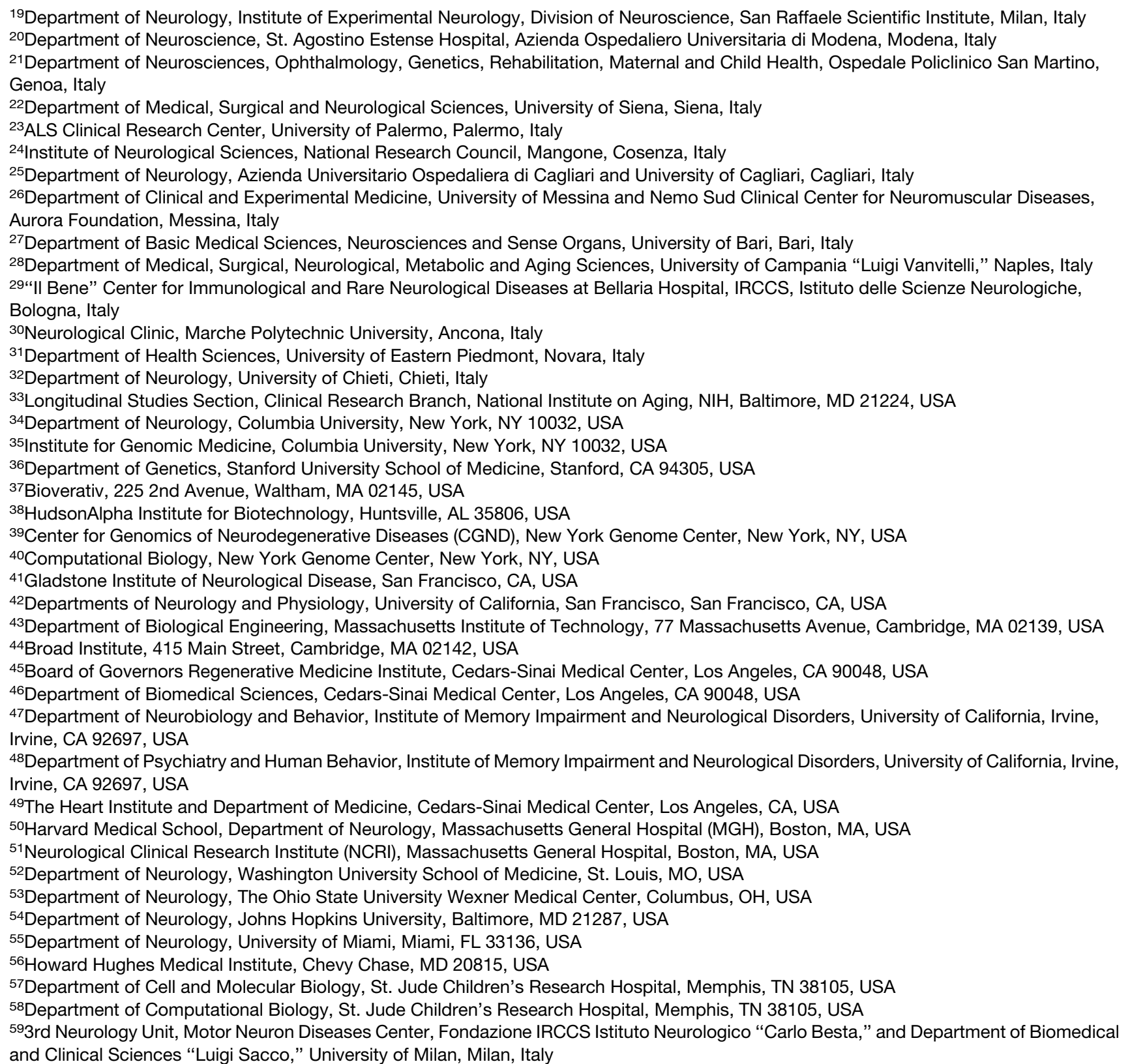


Francesco Landi, ${ }^{79}$ Robert Bowser, ${ }^{80}$ Stefan M. Pulst, ${ }^{81}$ John M. Ravits, ${ }^{82}$ Daniel J.L. MacGowan, ${ }^{83}$ Janine Kirby, ${ }^{84}$ Erik P. Pioro, ${ }^{85}$ Roger Pamphlett, ${ }^{86}$ James Broach, ${ }^{87}$ Glenn Gerhard, 88 Travis L. Dunckley, ${ }^{89}$ Christopher B. Brady, ${ }^{90,91}$ Neil W. Kowall, ${ }^{92}$ Juan C. Troncoso, ${ }^{93}$ Isabelle Le Ber, ${ }^{94}$ Kevin Mouzat, ${ }^{95,96}$ Serge Lumbroso, ${ }^{95,96}$

Terry D. Heiman-Patterson, ${ }^{97,98}$ Freya Kamel, ${ }^{99}$ Ludo Van Den Bosch, ${ }^{100,101}$ Robert H. Baloh, ${ }^{102}$ Tim M. Strom, ${ }^{103,104}$ Thomas Meitinger, ${ }^{103,104,105}$ Aleksey Shatunov, ${ }^{12}$ Kristel R. Van Eijk, ${ }^{10}$ Mamede de Carvalho, ${ }^{106,107}$ Maarten Kooyman, ${ }^{108}$ Bas Middelkoop, ${ }^{10}$ Matthieu Moisse, ${ }^{100,101}$ Russell L. McLaughlin, ${ }^{109}$ Michael A. Van Es, ${ }^{10}$ Markus Weber, ${ }^{110}$ Kevin B. Boylan,, ${ }^{111}$ Marka Van Blitterswijk, ${ }^{112}$ Rosa Rademakers, ${ }^{112}$ Karen E. Morrison, ${ }^{113}$ A. Nazli Basak, ${ }^{114}$ Jesús S. Mora, ${ }^{115}$ Vivian E. Drory, ${ }^{116}$ Pamela J. Shaw, ${ }^{84}$ Martin R. Turner, ${ }^{117}$ Kevin Talbot, ${ }^{117}$ Orla Hardiman, ${ }^{118}$ Kelly L. Williams, ${ }^{119}$ Jennifer A. Fifita, ${ }^{119}$ Garth A. Nicholson, ${ }^{119,120}$ Ian P. Blair, ${ }^{119}$ Guy A. Rouleau, ${ }^{121}$ Jesús Esteban-Pérez, ${ }^{122}$ Alberto García-Redondo, ${ }^{122}$ Ammar Al-Chalabi, ${ }^{12}$ Project MinE ALS Sequencing Consortium, Ekaterina Rogaeva, ${ }^{123}$ Lorne Zinman, ${ }^{124}$ Lyle W. Ostrow, ${ }^{54}$ Nicholas J. Maragakis, ${ }^{54}$ Jeffrey D. Rothstein, ${ }^{54}$

(Author list continued on next page)

${ }^{60}$ Neurology Unit, IRCCS Foundation Ca' Granda Ospedale Maggiore Policlinico, Milan, Italy

${ }^{61}$ Department of Neurosciences, University of Padova, Padova, Italy

62Genomic and Post-Genomic Center, IRCCS Mondino Foundation, Pavia, Italy

${ }^{63} \mathrm{ALS}$ Center, CHU Bretonneau, Tours University, Tours, France

${ }^{64}$ Department of Neurology, Helsinki University Hospital and Molecular Neurology Programme, Biomedicum, University of Helsinki, Helsinki FIN-02900, Finland

${ }^{65}$ Department of Pathology, University of Helsinki and Helsinki University Hospital, Helsinki, Finland

${ }^{66}$ Greater Manchester Neurosciences Centre, Salford Royal NHS Foundation Trust, Salford M6 8HD, UK

${ }^{67}$ Faculty of Human and Medical Sciences, University of Manchester, Manchester M13 9PT, UK

${ }^{68}$ Department of Clinical Neuroscience, Institute of Neurology, University College London, London NW3 2PG, UK

${ }^{69}$ Department of Molecular Neuroscience and Reta Lila Weston Laboratories, Institute of Neurology, University College London, Queen

Square House, London WC1N 3BG, UK

${ }^{70}$ Centre for Neuroscience and Trauma, Blizard Institute, Queen Mary University of London, NorthEast London and Essex Regional Motor

Neuron Disease Care Centre, London E1 2AT, UK

${ }^{71}$ Genomics Technology Group, Laboratory of Neurogenetics, National Institute on Aging, NIH, Porter Neuroscience Research Center, Bethesda, MD 20892, USA

72Department of Neurology, Emory University School of Medicine, Atlanta, GA 30322, USA

${ }^{73}$ Division of Brain Sciences, Department of Medicine, Imperial College London, London W120NN, UK

${ }^{74}$ Department of Clinical Genetics, Leiden University Medical Center, Leiden, the Netherlands

${ }^{75}$ Department of Neurogenetics and Neurology, Academic Medical Centre, Amsterdam, the Netherlands

${ }^{76}$ ALS-Neuromuscular Unit, Hospital General Universitario Gregorio Marañón, IISGM, Madrid, Spain

${ }^{77}$ Computational Biology Group, Laboratory of Neurogenetics, National Institute on Aging, NIH, Porter Neuroscience Research Center, Bethesda, MD 20892, USA

${ }^{78}$ Motor Neuron Disorders Unit, National Institute of Neurological Disorders and Stroke, NIH, Bethesda, MD 20892, USA

${ }^{79}$ Center for Geriatric Medicine, Department of Geriatrics, Neurosciences and Orthopedics, Catholic University of Sacred Heart, Rome 00168, Italy

${ }^{80}$ Division of Neurology, Barrow Neurological Institute, Phoenix, AZ, USA

${ }^{81}$ Department of Neurology, University of Utah School of Medicine, Salt Lake City, UT, USA

${ }^{82}$ Department of Neuroscience, University of California, San Diego, La Jolla, CA, USA

${ }^{83}$ Mount Sinai Beth Israel Hospital, Mount Sinai School of Medicine, New York, NY, USA

${ }^{84}$ Sheffield Institute for Translational Neuroscience (SITraN), University of Sheffield, Sheffield, UK

${ }^{85}$ Department of Neurology, Neuromuscular Center, Neurological Institute, Cleveland Clinic, Cleveland, OH, USA

${ }^{86}$ Discipline of Pathology, Brain and Mind Centre, The University of Sydney, 94 Mallett Street, Camperdown, NSW 2050, Australia

${ }^{87}$ Department of Biochemistry, Penn State College of Medicine, Hershey, PA, USA

${ }^{88}$ Department of Pathology, Penn State College of Medicine, Hershey, PA, USA

${ }^{89}$ Neurogenomics Division, Translational Genomics Research Institute, Phoenix, AZ, USA

${ }^{90}$ Research and Development Service, Veterans Affairs Boston Healthcare System, Boston, MA, USA

${ }^{91}$ Department of Neurology, Program in Behavioral Neuroscience, Boston University School of Medicine, Boston, MA, USA

${ }^{92}$ Neurology Service, VA Boston Healthcare System and Boston University Alzheimer's Disease Center, Boston, MA 02130, USA

${ }^{93}$ Departments of Pathology and Neurology, Johns Hopkins University School of Medicine, Baltimore, MD 21205, USA

${ }^{94}$ Sorbonne Universités, UPMC Univ Paris 06, Inserm, CNRS, Institut du Cerveau et la Moelle (ICM), Assistance Publique Hôpitaux de Paris (AP-HP) - Hôpital Pitié-Salpêtrière, Paris, France

${ }^{95}$ INM, University Montpellier, Montpellier, France

${ }^{96}$ Department of Biochemistry, CHU Nîmes, Nîmes, France

${ }^{97}$ Department of Neurology, Drexel University College of Medicine, Philadelphia, PA, USA

${ }^{98}$ Department of Neurology, Lewis Katz School of Medicine, Temple University, Philadelphia, PA, USA

${ }^{99}$ Epidemiology Branch, National Institute of Environmental Health Sciences, Durham, NC 27709, USA 
Zachary Simmons, ${ }^{125}$ Johnathan Cooper-Knock, ${ }^{84}$ Alexis Brice, ${ }^{94}$ Stephen A. Goutman, ${ }^{126}$ Eva L. Feldman, ${ }^{126}$

Summer B. Gibson, ${ }^{81}$ Franco Taroni, ${ }^{127}$ Antonia Ratti, ${ }^{5,6}$ Cinzia Gellera, ${ }^{127}$ Philip Van Damme, ${ }^{100,101,128}$

Wim Robberecht, ${ }^{100,101,128}$ Pietro Fratta, ${ }^{14}$ Mario Sabatelli, ${ }^{129}$ Christian Lunetta, ${ }^{130}$ Albert C. Ludolph, ${ }^{131}$

Peter M. Andersen, ${ }^{132}$ Jochen H. Weishaupt, ${ }^{131}$ William Camu, ${ }^{133}$ John Q. Trojanowski, ${ }^{134}$ Vivianna M. Van Deerlin, ${ }^{134}$

Robert H. Brown, Jr., ${ }^{2}$ Leonard H. van den Berg, ${ }^{10}$ Jan H. Veldink, ${ }^{10}$ Matthew B. Harms, ${ }^{34}$ Jonathan D. Glass, ${ }^{72}$

David J. Stone, ${ }^{135,137}$ Pentti Tienari, ${ }^{64,137}$ Vincenzo Silani, ${ }^{5,6,137}$ Adriano Chiò, ${ }^{17,136,137}$ Christopher E. Shaw, ${ }^{12,137}$

Bryan J. Traynor, ${ }^{1,54,137, *}$ and John E. Landers ${ }^{2,137,138, *}$

$100 \mathrm{KU}$ Leuven - University of Leuven, Department of Neurosciences, Experimental Neurology and Leuven Research Institute for Neuroscience and Disease (LIND), B-3000 Leuven, Belgium

${ }^{101} \mathrm{VIB}$, Center for Brain and Disease Research, Laboratory of Neurobiology, Leuven, Belgium

${ }^{102}$ Department of Neurology, Cedars-Sinai Medical Center, Los Angeles, CA, USA

${ }^{103}$ Institute of Human Genetics, Technische Universität München, Munich, Germany

${ }^{104}$ Institute of Human Genetics, Helmholtz Zentrum München, German Research Center for Environmental Health, Neuherberg, Germany

${ }^{105}$ Munich Cluster for Systems Neurology (SyNergy), Munich, Germany

${ }^{106}$ Institute of Physiology, Institute of Molecular Medicine, Faculty of Medicine, University of Lisbon, Lisbon, Portugal

${ }^{107}$ Department of Neurosciences, Hospital de Santa Maria-CHLN, Lisbon, Portugal

${ }_{108}$ SURFsara, Amsterdam, the Netherlands

${ }^{109}$ Population Genetics Laboratory, Smurfit Institute of Genetics, Trinity College Dublin, Dublin, Republic of Ireland

${ }_{110}$ Neuromuscular Diseases Center/ALS Clinic, Kantonsspital St. Gallen, St. Gallen, Switzerland

${ }^{111}$ Department of Neurology, Mayo Clinic Florida, Jacksonville, FL 32224, USA

${ }^{112}$ Department of Neuroscience, Mayo Clinic, Jacksonville, FL, USA

${ }^{113}$ Faculty of Medicine, University of Southampton, Southampton, UK

${ }^{114}$ Suna and Inan Kırac Foundation, Neurodegeneration Research Laboratory, Bogazici University, Istanbul, Turkey

${ }^{115}$ ALS Unit/Neurology, Hospital San Rafael, Madrid, Spain

${ }^{116}$ Department of Neurology, Tel-Aviv Sourasky Medical Centre, Tel-Aviv, Israel

${ }^{117}$ Nuffield Department of Clinical Neurosciences, University of Oxford, Oxford, UK

${ }^{118}$ Academic Unit of Neurology, Trinity Biomedical Sciences Institute, Trinity College Dublin, Dublin, Republic of Ireland

${ }^{119}$ Centre for MND Research, Faculty of Medicine and Health Sciences, Macquarie University, Sydney, NSW 2109, Australia

${ }^{120}$ ANZAC Research Institute, Concord Hospital, University of Sydney, Sydney, NSW 2139, Australia

${ }^{121}$ Montreal Neurological Institute, Department of Neurology and Neurosurgery, McGill University, Montreal, QC, Canada

122Unidad de ELA, Instituto de Investigación Hospital 12 de Octubre de Madrid, SERMAS, and Centro de Investigación Biomédica en Red de Enfermedades Raras (CIBERER U-723), Madrid, Spain

${ }^{123}$ Tanz Centre for Research of Neurodegenerative Diseases, Division of Neurology, Department of Medicine, University of Toronto, Toronto, ON M5S 3H2, Canada

${ }^{124}$ Division of Neurology, Department of Internal Medicine, Sunnybrook Health Sciences Centre, University of Toronto, Toronto, ON M4N 3M5, Canada

${ }^{125}$ Department of Neurology, Penn State Hershey Medical Center, Hershey, PA, USA

${ }^{126}$ Department of Neurology, University of Michigan, Ann Arbor, MI, USA

${ }^{127}$ Unit of Genetics of Neurodegenerative and Metabolic Diseases, Fondazione IRCCS Istituto Neurologico "Carlo Besta," Milan 20133, Italy

${ }^{128}$ University Hospitals Leuven, Department of Neurology, Leuven, Belgium

${ }^{129}$ Centro Clinico NeMO, Institute of Neurology, Catholic University, Largo F. Vito 1, 00168 Rome, Italy

${ }^{130 N E u r o M u s c u l a r ~ O m n i c e n t e r ~(N E M O), ~ S e r e n a ~ O n l u s ~ F o u n d a t i o n, ~ M i l a n, ~ I t a l y ~}$

${ }^{131}$ Neurology Department, Ulm University, Albert-Einstein-Allee 11, 89081 Ulm, Germany

132Department of Pharmacology and Clinical Neuroscience, Umeå University, Umeå SE-90185, Sweden

${ }^{133}$ ALS Center, CHU Gui de Chauliac, University of Montpellier, Montpellier, France

${ }^{134}$ Department of Pathology and Laboratory Medicine, University of Pennsylvania, Philadelphia, PA, USA

135Genetics and Pharmacogenomics, MRL, Merck \& Co., Inc., West Point, PA 19486, USA

${ }^{136}$ Neuroscience Institute of Torino, Turin 10124, Italy

${ }^{137}$ These authors contributed equally

${ }^{138}$ Lead Contact

*Correspondence: bryan.traynor@nih.gov (B.J.T.), john.landers@umassmed.edu (J.E.L.)

https://doi.org/10.1016/j.neuron.2018.02.027

(Hirtz et al., 2007). Furthermore, the number of ALS cases across the globe will increase to nearly 400,000 in 2040 , predominantly due to aging of the population (Arthur et al., 2016). This increase is anticipated to place an enormous socioeconomic burden on global healthcare systems, in particular because the annual healthcare cost per patient with ALS is among the highest for any neurological disease (Gladman and Zinman, 2015).
Approximately $10 \%$ of ALS cases display a family history (FALS), whereas the remaining $90 \%$ of ALS cases are sporadic (SALS) in nature. Driven in large part by advances in genotyping and sequencing technology, the genetic etiology of two-thirds of FALS cases and about $10 \%$ of SALS cases is now known (Chia et al., 2018; Renton et al., 2014). Mutations in SOD1 were the first identified cause of ALS (Rosen et al., 1993), contributing to $\sim 20 \%$ of FALS and $\sim 2 \%$ of SALS. More recently, pathogenic 
A

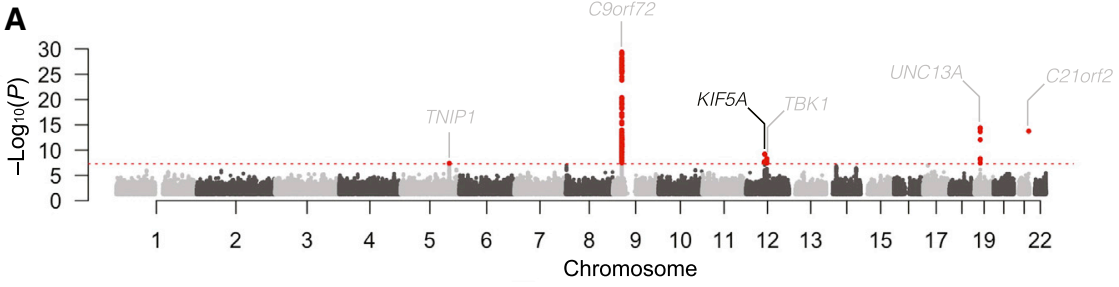

B

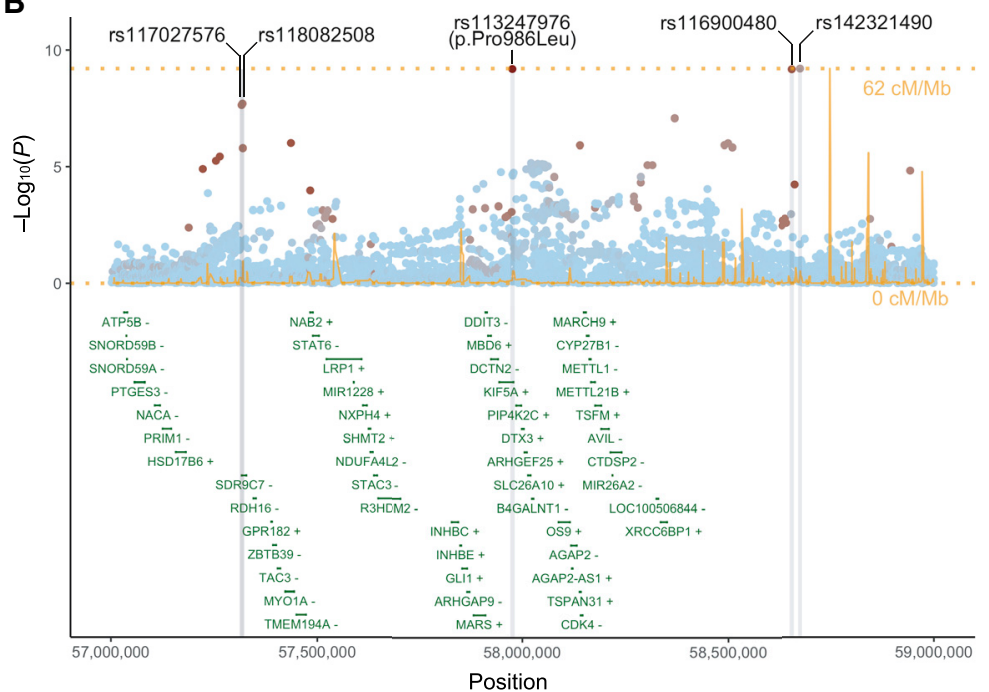

Figure 1. Identification of Association between KIF5A Locus and ALS Risk through GWAS

(A) Manhattan plot showing $p$ values from the discovery set GWAS. Analysis of a combined set of 20,806 cases and 59,804 controls is shown. The dashed red line denotes the threshold for genomewide significance after multiple test correction $\left(p<5.0 \times 10^{-8}\right)$. Five previously reported ALSassociated loci are labeled in gray and one novel loci, containing the KIF5A gene, is labeled in black. (B) Regional association plot of the KIF5A locus. Recombination rates are from HapMap phase 2 European ancestry samples. The $R^{2}$ pattern is based on the rs113247976 SNP using 85 European ancestry samples (CEU) from the November 2010 release of the 1000 Genomes Project data-

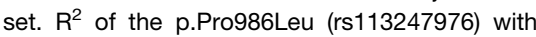
additional SNPs achieving genome-wide significance was 0.544 (rs117027576), 0.544 (rs118082508), 0.741 (rs116900480), and 0.347 (rs142321490).

genes continue to provide valuable insight into ALS pathogenesis. For example, the product of the risk factor $T B K 1$ is known to interact with the product of ALS-associated gene OPTN, further solidifying the role of autophagy

hexanucleotide repeat expansions located within the first intron of the C9orf72 gene on chromosome 9p21 were identified as the most common cause of both FALS ( 40\%) and SALS ( 7\%) (DeJesus-Hernandez et al., 2011; Renton et al., 2011). Interestingly, this repeat expansion contributes to $\sim 10 \%$ of all frontotemporal dementia (FTD) cases, thus genetically explaining much of the overlap between these clinical syndromes (Majounie et al., 2012). As a result of these major discoveries, there are several ongoing efforts toward directed silencing of these mutant genes, which could result in a therapeutic treatment for up to $10 \%$ of all ALS cases and for a similar portion of FTD cases.

In addition to the insights provided by each novel ALS gene, the collective knowledge gained from genetic factors provides a more comprehensive understanding of the interacting pathways underlying motor neuron degeneration. For example, the identification of ALS genes has revealed at least three pathways believed to contribute to the development of ALS: (1) RNA metabolism (based on the observation of mutations in C9orf72, TDP43, FUS, HNRNPA1, and MATR3), (2) protein homeostasis (UBQLN2, VCP, OPTN, and VAPB), and (3) cytoskeletal dynamics (PFN1, TUBA4A, and DCTN1) (Chia et al., 2018; Robberecht and Eykens, 2015; Taylor et al., 2016). Understanding the mechanisms leading to disease pathogenesis again provides targets for therapeutic intervention that may be applicable to all forms of ALS.

Due to the decreased accessibility of multiple affected family members with unknown genetic etiology, there has been an increased focus on the identification of ALS-associated genes with moderate to low impact. Despite their lower effect, such and protein homeostasis in disease development (Cirulli et al., 2015; Freischmidt et al., 2015; Maruyama et al., 2010; Morton et al., 2008). Similarly, the risk factor NEK1, identified through a rare variant burden analysis (RVB) of index FALS (i.e., one affected sample per family), is a known binding partner of C21orf2, an ALS risk factor found through genome-wide association studies (GWASs) (Cirulli et al., 2015; Kenna et al., 2016; Malovannaya et al., 2011; van Rheenen et al., 2016). The interaction of these two proteins is required for efficient DNA damage repair (Fang et al., 2015), a pathway that is becoming increasingly implicated as a contributing factor in ALS and other neurodegenerative diseases (Coppedè and Migliore, 2015; Lopez-Gonzalez et al., 2016; Madabhushi et al., 2014; Wang et al., 2013).

\section{RESULTS}

GWASs Identify KIF5A as a Novel ALS-Associated Gene To identify new susceptibility loci operating in ALS, we undertook a large-scale GWAS involving 12,663 patients diagnosed with ALS and 53,439 control subjects (Tables S1 and S2). Our data were then incorporated into a meta-analysis with a recently published GWAS involving 12,577 ALS cases and 23,475 control subjects (van Rheenen et al., 2016). After imputation and quality-control measures (see STAR Methods and Figure S1 for the workflow and Figure S2 for the multidimensional scaling plot), 10,031,630 genotyped and imputed variants from 20,806 ALS cases and 59,804 control samples were available for association analysis (Figure 1A). Quantile-quantile plots did not show evidence of significant population stratification $\left(\lambda_{1000}=1.001\right.$; 
Figure S3). SNPs achieving genome-wide significance $(p<5.0 \times$ $\left.10^{-8}\right)$ are listed in Tables 1 and S3 and suggestive loci with SNPs associated at $p<5.0 \times 10^{-7}$ are listed in Table S4.

Our analysis revealed five previously identified loci that achieved genome-wide significance (loci including TNIP1, C9orf72, TBK1, UNC13A, and C21orf2) (Benyamin et al., 2017; Laaksovirta et al., 2010; Shatunov et al., 2010; van Es et al., 2009; van Rheenen et al., 2016). In addition, we observed a strong association signal for five SNPs in linkage disequilibrium on chromosome 12q14.1 that reached genome-wide statistical significance spanning a region of several hundred kilobases (Table 1; Figure 1B). Of the five SNPs, two of them resided in close proximity to each other within a large intergenic region and two in proximity to short-chain dehydrogenase/reductase family 9C member 7 (SDR9C7), a gene expressed primarily in skin. However, one SNP (rs113247976) results in a p.Pro986Leu coding change within the kinesin family member $5 A$ (KIF5A) gene $\left(p=6.4 \times 10^{-10}\right.$, odds ratio $[\mathrm{OR}]=1.38,95 \% \mathrm{Cl}=1.24-1.53$; Figure 2). The case:control allele frequencies for the combined discovery cohort were $2.07 \%: 1.55 \%$ and genotype counts were 5: 529: 12,043 to 7: 786: 22,682 (homozygotes alternative allele: heterozygotes: homozygous reference allele; Figure 2). Calculations based on our cohort size as well as the OR and allele frequency of rs113247976 result in an $\sim 99.5 \%$ power to detect this as an ALS-associated SNP.

\section{Rare Variant Burden Analysis Identifies KIF5A as an ALS Gene}

In an independent line of investigation, we attempted to identify novel ALS genes through exome-wide RVB. In brief, RVB compares the frequency of variants within each gene below a userdefined frequency threshold in a case-control cohort. As the last two ALS-associated genes identified by this methodology (TBK1 and NEK1) displayed an increased frequency of loss-offunction (LOF) variants, we focused our initial analysis on such variants (consisting of nonsense and predicted splice altering) (Cirulli et al., 2015; Freischmidt et al., 2015; Kenna et al., 2016).

Toward this end, we performed RVB testing for association of LOF variants in a cohort of 1,138 index FALS cases and 19,494 controls, after applying quality-control filters (Experimental Model and Subject Details; Figure S4; Tables S5 and S6). Genes displaying $\mathrm{p}<5.0 \times 10^{-4}$ are shown in Table 2 . The previously identified ALS genes, TBK1 $\left(p=5.58 \times 10^{-7}\right.$, OR $=15.11$, $95 \% \mathrm{Cl}=5.81-38.69)$ and NEK1 $\left(\mathrm{p}=1.68 \times 10^{-6}, \mathrm{OR}=6.64\right.$, $95 \% \mathrm{Cl}=3.32-12.51$ ), yielded strong associations with ALS, reaching exome-wide significance (Figure 3). In addition, we observed a single novel gene reaching exome-wide significance, KIF5A ( $\mathrm{p}=5.55 \times 10^{-7}$; OR = 32.07, 95\% Cl = 9.05-135.27). Within this gene, we observed 6 LOF variants in our 1,138 cases $(0.53 \%)$ compared to 3 such variants in our comparison cohort of 19,494 controls $(0.015 \%$; Table 2$)$. There was no evidence of genomic inflation $(\lambda=0.93$; Figure S5), sequencing center or other sub-cohort bias (Figure S6), or call rate bias (Figure S7) in our analysis. Of the index FALS cases carrying KIF5A LOF mutations, we obtained DNA from two siblings of the proband carrying a c.2993-3C>T, exon $27-5^{\prime}$ splice junction variant and from a sibling of a different proband carrying a c. $3020+2 T>A$, exon $27-3^{\prime}$ splice junction variant. These variants segregated with disease within each of these families. Sanger sequencing validated all identified LOF variants containing samples in the discovery set and affected relatives.

Interestingly, when we investigated the location of the six ALSassociated variants present in KIF5A, all occurred within a $34 \mathrm{bp}$ stretch of DNA and were predicted to affect splicing of exon 27 , which encodes amino acids 998-1007 (Table 3; Figure 4A). Five of the six variants were located on sequential base pairs on the $3^{\prime}$ end of the exon, whereas one was located on the $5^{\prime}$ end of the exon. We used the application ASSEDA (Automated Splice Site and Exon Definition Analyses) to predict any mutant mRNA splice isoforms resulting from these variants (Tompson et al., 2007). This algorithm was chosen as it is known to have high performance in splice prediction (Caminsky et al., 2014). ASSEDA predicted a complete skipping of exon 27 for all variants, yielding a transcript with a frameshift at coding amino acid 998, the deletion of the normal C-terminal 34 amino acids of the cargo-binding domain, and the extension of an aberrant 39 amino acids to the $\mathrm{C}$ terminus (Table 3; Figures 4B and $4 \mathrm{C}$ ). The presence of transcripts with skipped exon 27 was demonstrated by performing RT-PCR in two patients carrying exon 27 - $3^{\prime}$ splice junction variants (c.3020+2T>A and c. $3020+1 \mathrm{G}>\mathrm{A}$ ) using RNA from lymphoblasts and peripheral blood mononuclear cells, respectively. This splice form was not detected in four control lines (Figure 4D). Sequence analysis of the smaller RT-PCR products obtained from the patient cells confirmed the exon 26-28 splicing event. Material for RT-PCR was not available for any other patient carrying a KIF5A LOF variant.

Our initial RVB was restricted to single nucleotide variants due to the limited sensitivity and comparatively high false-positive rates associated with identifying small insertions and deletions (indels) within exome sequencing data (Fang et al., 2014). Based on our discovery of increased LOF variants within KIF5A, we reevaluated this region for the presence of indels. Our analysis revealed two $(0.026 \%)$ indels within our cohort of 1,138 FALS cases, compared to zero (0\%) indels among 19,494 control samples. Both of these indels (p.Asp996fs and p.Asn999fs) resulted in a frameshift of the KIF5A protein coding sequence, and were located close to the splice junction variants that we previously observed to cause skipping of exon 27 , resulting in a frameshift at amino acid 998 (Table 3). Sanger sequencing confirmed the presence of both indels. Combining the results of the single nucleotide and indel variant analysis yielded a highly significant $p$ of $3.8 \times 10^{-9}(\mathrm{OR}=41.16,95 \% \mathrm{Cl}=12.61-167.57)$. We failed to detect any signals of RVB association for rare missense variants across KIF5A or within any sub-domain of the gene (Table S8).

\section{Replication Analysis of rs113247976 and LOF Variants in KIF5A}

Given the strong signal of the missense variant identified by our GWAS (p.Pro986Leu, rs113247976) and its close proximity to the LOF variants identified by our RVB (amino acids 996999), we attempted to replicate its association with ALS by analyzing additional cohorts. To accomplish this, we evaluated this variant in a cohort of 4,159 ALS cases and 18,650 controls that were non-overlapping with our GWAS discovery analysis 


\begin{tabular}{|c|c|c|c|c|c|c|c|c|c|c|c|c|c|c|c|}
\hline \multicolumn{4}{|c|}{ SNP Information } & \multicolumn{4}{|c|}{ Present Study (8,229 Cases/36,329 Controls) } & \multicolumn{4}{|c|}{$\begin{array}{l}\text { Van Rheenen et al. (12,577 Cases/23,475 } \\
\text { Controls) }\end{array}$} & \multicolumn{4}{|c|}{$\begin{array}{l}\text { Combined Discovery Set ( } 20,806 \text { Cases/ } \\
59,804 \text { Controls) }\end{array}$} \\
\hline SNP & Chr & Position & Gene & $\begin{array}{l}\text { Case } \\
\text { MAF }\end{array}$ & $\begin{array}{l}\text { Control } \\
\text { MAF }\end{array}$ & OR $[95 \% \mathrm{Cl}]$ & $\mathrm{p}$ & $\begin{array}{l}\text { Case } \\
\text { MAF }\end{array}$ & $\begin{array}{l}\text { Control } \\
\text { MAF }\end{array}$ & OR $[95 \% \mathrm{Cl}]$ & $\mathrm{p}$ & $\begin{array}{l}\text { Case } \\
\text { MAF }\end{array}$ & $\begin{array}{l}\text { Control } \\
\text { MAF }\end{array}$ & OR [95\% Cl] & $\mathrm{p}$ \\
\hline \multicolumn{16}{|l|}{ Novel Loci } \\
\hline rs117027576 & 12 & $57,316,603$ & KIF5A & $1.55 \%$ & $1.27 \%$ & $\begin{array}{l}1.45 \\
{[1.20-1.76]}\end{array}$ & $1.1 \times 10^{-4}$ & $1.98 \%$ & $1.59 \%$ & $\begin{array}{l}1.33 \\
{[1.16-1.53]}\end{array}$ & $4.3 \times 10^{-5}$ & $1.81 \%$ & $1.40 \%$ & $\begin{array}{l}1.37 \\
{[1.23-1.54]}\end{array}$ & $2.3 \times 10^{-8}$ \\
\hline rs118082508 & 12 & $57,318,819$ & KIF5A & $1.56 \%$ & $1.28 \%$ & $\begin{array}{l}1.45 \\
{[1.20-1.76]}\end{array}$ & $1.0 \times 10^{-4}$ & $1.98 \%$ & $1.60 \%$ & $\begin{array}{l}1.33 \\
{[1.16-1.53]}\end{array}$ & $3.8 \times 10^{-5}$ & $1.81 \%$ & $1.41 \%$ & $\begin{array}{l}1.37 \\
{[1.23-1.54]}\end{array}$ & $2.0 \times 10^{-8}$ \\
\hline rs113247976* & 12 & $57,975,700$ & KIF5A & $1.83 \%$ & $1.42 \%$ & $\begin{array}{l}1.46 \\
{[1.23-1.74]}\end{array}$ & $9.2 \times 10^{-6}$ & $2.14 \%$ & $1.70 \%$ & $\begin{array}{l}1.33 \\
{[1.17-1.52]}\end{array}$ & $1.1 \times 10^{-5}$ & $2.02 \%$ & $1.53 \%$ & $\begin{array}{l}1.38 \\
{[1.24-1.53]}\end{array}$ & $6.4 \times 10^{-10}$ \\
\hline rs116900480 & 12 & $58,656,105$ & KIF5A & $1.75 \%$ & $1.46 \%$ & $\begin{array}{l}1.42 \\
{[1.21-1.68]}\end{array}$ & $1.9 \times 10^{-5}$ & $2.08 \%$ & $1.66 \%$ & $\begin{array}{l}1.34 \\
{[1.18-1.53]}\end{array}$ & $7.1 \times 10^{-6}$ & $1.95 \%$ & $1.54 \%$ & $\begin{array}{l}1.37 \\
{[1.24-1.52]}\end{array}$ & $6.6 \times 10^{-10}$ \\
\hline rs142321490 & 12 & $58,676,132$ & KIF5A & $1.79 \%$ & $1.48 \%$ & $\begin{array}{l}1.43 \\
{[1.21-1.68]}\end{array}$ & $1.5 \times 10^{-5}$ & $2.08 \%$ & $1.66 \%$ & $\begin{array}{l}1.34 \\
{[1.18-1.53]}\end{array}$ & $8.0 \times 10^{-6}$ & $1.97 \%$ & $1.55 \%$ & $\begin{array}{l}1.37 \\
{[1.24-1.52]}\end{array}$ & $6.1 \times 10^{-10}$ \\
\hline \multicolumn{16}{|c|}{ Previously Published Loci } \\
\hline rs10463311 & 5 & $150,410,835$ & TNIP1 & $73.19 \%$ & $74.84 \%$ & $\begin{array}{l}0.94 \\
{[0.89-0.98]}\end{array}$ & $7.8 \times 10^{-3}$ & $73.34 \%$ & $75.79 \%$ & $\begin{array}{l}0.91 \\
{[0.87-0.94]}\end{array}$ & $8.5 \times 10^{-7}$ & $73.28 \%$ & $75.21 \%$ & $\begin{array}{l}0.92 \\
{[0.89-0.95]}\end{array}$ & $4.0 \times 10^{-8}$ \\
\hline rs3849943 & 9 & $27,543,382$ & C9orf72 & $71.79 \%$ & $76.31 \%$ & $\begin{array}{l}0.84 \\
{[0.80-0.88]}\end{array}$ & $1.4 \times 10^{-12}$ & $72.78 \%$ & $76.5 \%$ & $\begin{array}{l}0.83 \\
{[0.80-0.87]}\end{array}$ & $4.0 \times 10^{-19}$ & $72.39 \%$ & $76.38 \%$ & $\begin{array}{l}0.84 \\
{[0.81-0.86]}\end{array}$ & $3.8 \times 10^{-30}$ \\
\hline rs74654358 & 12 & $64,881,967$ & TBK1 & $3.77 \%$ & $4.01 \%$ & $\begin{array}{l}1.20 \\
{[1.07-1.34]}\end{array}$ & $1.6 \times 10^{-3}$ & $5.12 \%$ & $4.61 \%$ & $\begin{array}{l}1.23 \\
{[1.13-1.34]}\end{array}$ & $7.7 \times 10^{-7}$ & $4.59 \%$ & $4.25 \%$ & $\begin{array}{l}1.22 \\
{[1.14-1.30]}\end{array}$ & $4.7 \times 10^{-9}$ \\
\hline rs12973192 & 19 & $17,753,239$ & UNC13A & $67.62 \%$ & $69.37 \%$ & $\begin{array}{l}0.86 \\
{[0.82-0.91]}\end{array}$ & $1.3 \times 10^{-8}$ & $64.52 \%$ & $66.00 \%$ & $\begin{array}{l}0.9 \\
{[0.87-0.93]}\end{array}$ & $2.4 \times 10^{-8}$ & $65.75 \%$ & $68.05 \%$ & $\begin{array}{l}0.89 \\
{[0.86-0.91]}\end{array}$ & $3.9 \times 10^{-15}$ \\
\hline rs75087725 & 21 & $45,753,117$ & C21orf2 & $0.70 \%$ & $0.46 \%$ & $\begin{array}{l}1.99 \\
{[1.44-2.75]}\end{array}$ & $2.2 \times 10^{-5}$ & $1.83 \%$ & $1.27 \%$ & $\begin{array}{l}1.61 \\
{[1.39-1.87]}\end{array}$ & $8.7 \times 10^{-11}$ & $1.38 \%$ & $0.78 \%$ & $\begin{array}{l}1.67 \\
{[1.46-1.91]}\end{array}$ & $1.8 \times 10^{-14}$ \\
\hline
\end{tabular}

Position is based on Human Genome Assembly build 37 . Nearest gene or previously published gene names are included. Chr, chromosome; MAF, minor allele frequency; OR, odds ratio; $95 \%$ Cl, confidence interval; *rs113247976 represents the p.Pro986Leu variant in KIF5A (GenBank: NM_004984.2). 


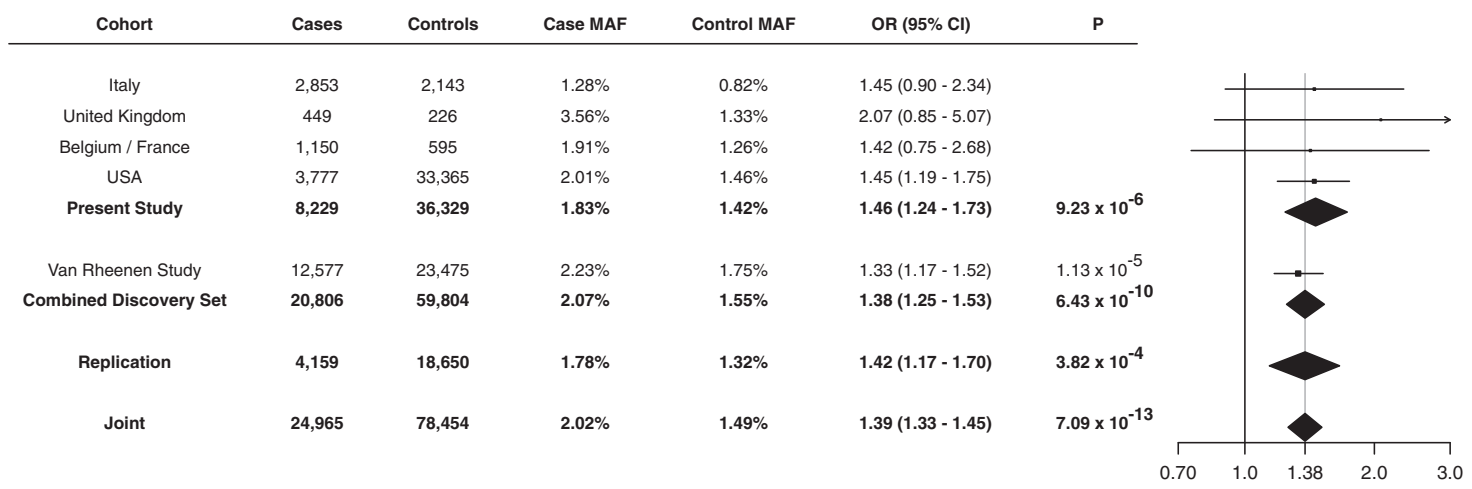

Figure 2. Discovery and Replication of the Association of the KIF5A p.Pro986Leu (rs113247976) Variant with ALS

Analysis of the p.Pro986Leu (rs113247976) variant within each of the described cohorts is shown. Allelic association for all subcohorts was analyzed by logistic regression followed by a fixed-effects meta-analysis. The Forest plot (right) displays the distribution of OR estimates across study cohorts with the vertical gray line denoting the OR estimated under the meta-analysis, and with the width of the horizontal lines and the diamonds corresponding to $95 \%$ confidence intervals.

(STAR Methods; Figure S8). This included non-overlapping samples from our RVB (673 FALS and 17,696 controls). Analysis of the cohort revealed an allele frequency of $1.78 \%$ in cases and $1.32 \%$ in controls ( $r$ 113247976, $\mathrm{p}=3.82 \times 10^{-4}, \mathrm{OR}=1.42$, $95 \% \mathrm{Cl}=1.17-1.70)$, thereby replicating the association of the original GWAS. A meta-analysis of the GWAS and replication cohort ( $n=24,965$ cases and 78,454 controls) yielded a highly significant $\mathrm{p}$ of $7.09 \times 10^{-13}(\mathrm{OR}=1.39,95 \% \mathrm{Cl}=1.33-1.45$; Figure 2). These results support the association of KIF5A p.Pro986Leu with ALS. However, at this point we cannot definitely state that the missense variant is the primary risk factor, as we cannot rule out other variants in linkage disequilibrium.

We next performed mutational screening of KIF5A in an additional cohort of 9,046 ALS cases that had not been included in our original RVB. This revealed three additional carriers of C-terminal variants. One sporadic patient harbored an exon 26 frameshift mutation ( $p . A s n 997 \mathrm{fs}$ ) and a second sporadic patient harbored an exon 27 splice-altering mutation (c.2993-1G>A; Table 3). The third patient carried a p.Arg1007Lys (c.3020G>A) mutation and had a familial history of ALS. This mutation was also observed in FALS patients from our RVB; however, a comparison of 240,715 common variant sites between the two patients failed to reveal a familial relationship (Experimental Model and Subject Details). Additionally, one patient was observed to carry a predicted splice-altering variant proximal to exon 3 (c.291+5G>A). However, this variant was not supported as creating an aberrant transcript by ASSEDA. The cohort used for this analysis was comprised mainly of sporadic ALS cases. LOF variants were not observed in a follow-up panel of 1,955 controls. Comparison of the LOF variants in sporadic patients $(2 / 9,046$ cases, $0.022 \%)$ with either the 1,955 replication controls or all controls analyzed in this study $(21,449$ controls) yielded insignificant $p$ values $(0.868$ and 0.423 , respectively). Interestingly, the frequency of LOF variants in sporadic cases is lower than that observed in our original FALS cohort (0.703\%), suggesting that KIF5A LOF variants display a high penetrance. Furthermore, the rate of LOF variants reported in the Exome Aggregation Consortium (ExAC) database is lower than we observed in the control samples used in our discovery cohort (0.007\% versus $0.015 \%)$.

\section{ALS-Associated Mutations in KIF5A Are Distinct from SPG10/CMT2 Mutations}

Missense mutations within KIF5A are a known cause of hereditary spastic paraparesis (spastic paraplegia type 10, autosomal dominant; OMIM: 604187) and of Charcot-Marie-Tooth disease type 2 (CMT2) (Crimella et al., 2012; Jennings et al., 2017; Liu et al., 2014; Reid et al., 2002). Although SPG10 and CMT2 share

\begin{tabular}{lllll}
\hline \multicolumn{1}{l}{ Table 2. Top ALS Associations Identified through RVB of FALS and Control Exome Sequencing Results } \\
\hline Gene & FALS & Control & OR & p \\
\hline KIF5A & $6(0.53 \%)$ & $3(0.02 \%)$ & $32.07(9.05-135.27)$ & $5.55 \times 10^{-7}$ \\
TBK1 & $8(0.70 \%)$ & $9(0.05 \%)$ & $15.11(5.81-38.69)$ & $5.58 \times 10^{-7}$ \\
NEK1 & $12(1.05 \%)$ & $32(0.16 \%)$ & $6.64(3.32-12.51)$ & $1.68 \times 10^{-6}$ \\
CALHM2 & $7(0.62 \%)$ & $9(0.05 \%)$ & $12.13(4.47-31.79)$ & $9.19 \times 10^{-6}$ \\
COL14A1 & $8(0.70 \%)$ & $16(0.08 \%)$ & $8.04(3.32-18.08)$ & $2.72 \times 10^{-5}$ \\
AK1 & $10(0.88 \%)$ & $34(0.17 \%)$ & $5.37(2.55-10.41)$ & $5.62 \times 10^{-5}$ \\
ATRN & $5(0.44 \%)$ & $9(0.05 \%)$ & $11.06(3.57-31.02)$ & $1.66 \times 10^{-4}$ \\
VLDLR & $5(0.44 \%)$ & $9(0.05 \%)$ & $10.87(3.51-30.43)$ & $1.79 \times 10^{-4}$ \\
FUS & $4(0.35 \%)$ & $4(0.02 \%)$ & $16.53(4.25-64.33)$ & $2.08 \times 10^{-4}$ \\
ZMYND12 & $6(0.53 \%)$ & $12(0.06 \%)$ & $7.92(2.86-19.96)$ & $2.61 \times 10^{-4}$ \\
\hline
\end{tabular}




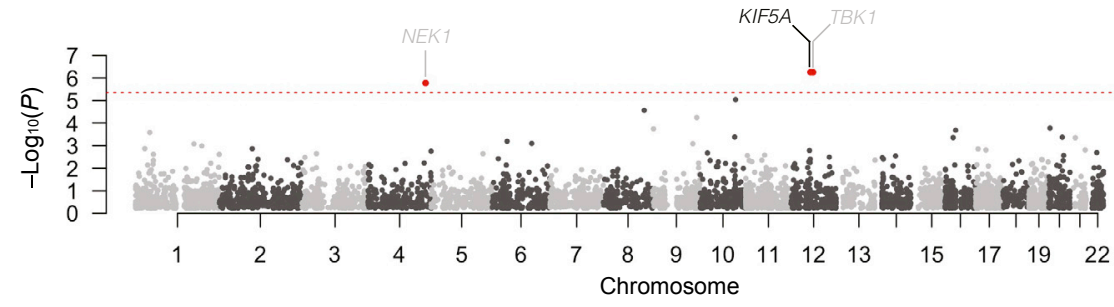

Figure 3. Identification of Association between KIF5A and ALS Risk through Rare Variant Burden Analysis of Exome Sequencing

Manhattan plot showing gene-level $p$ values from an exome-wide rare variant burden analysis. Analyses of 1,138 index FALS cases versus 19,494 controls were restricted to rare LOF variants (splice altering/nonsense, MAF $<0.001$ ). A minimum of three LOF variant carriers were required for analysis. The dashed red line denotes the threshold for exome-wide significance after correction for 11,472 genes $\left(4.36 \times 10^{-6}\right)$. Previously reported (gray) and novel (black) genes exhibiting a significant excess of rare LOF variants in patients are shown.

clinical features with ALS, a careful examination of the clinical records of the ALS cases with LOF mutations in KIF5A ruled out misdiagnosis. Furthermore, we detected no variants previously associated with SPG10 or CMT2 in our FALS cohort (Liu et al., 2014).

To further elucidate genotype-phenotype relationships, we evaluated the location of mutations within KIF5A. Interestingly, mutations contributing to SPG10 and to CMT2 are almost exclusively missense changes and are located in the $\mathrm{N}$-terminal motor domain (amino acids 9-327) of KIF5A (Figure 5). In contrast, the mutations identified as contributing to ALS are found predominantly in the C-terminal cargo-binding region of KIF5A (amino acids 907-1032) with the highly penetrant FALS mutations showing LOF. These results indicate that the functional domain mutated in KIF5A dictates the clinical phenotype, resulting in distinct yet overlapping neurodegenerative diseases.

\section{Patients with KIF5A LOF Mutations Display Younger Age} at Onset and Longer Survival

To establish the existence of any commonalities between patients with LOF mutations in the C-terminal region of KIF5A, we evaluated their clinical phenotype. Cases with LOF mutations exhibited a median age of onset at 46.5 years $(n=19$; Table S7). This is lower than the age of onset reported for ALS in epidemiological studies (65.2 years, interquartile range 56.0-72.2) (Ahmeti et al., 2013). Interestingly, we also observed an increased disease duration (survival) in patients harboring these LOF mutations. The median survival time of ALS patients is 20-36 months (Ahmeti et al., 2013). In contrast, cases with LOF mutations exhibited a median survival of nearly 10 years (117 months, $\mathrm{n}=17$; Table S7). ALS patients with symptom onset before 40 years of age have been shown to have longer survival, often exceeding 10 years (Chiò et al., 2009). In contrast,

\begin{tabular}{|c|c|c|c|c|c|c|c|c|c|c|}
\hline Position & Variant & Exon & cDNA & Description & $\begin{array}{l}\text { Predicted } \\
\text { Exon } \\
\text { Skipping }\end{array}$ & Gender & $\begin{array}{l}\text { Age of } \\
\text { Onset } \\
\text { (Years) }\end{array}$ & $\begin{array}{l}\text { Site of } \\
\text { Onset }\end{array}$ & $\begin{array}{l}\text { Survival } \\
\text { (Months) }\end{array}$ & $\begin{array}{l}\text { Alive } \\
\text { (Yes/No) }\end{array}$ \\
\hline \multicolumn{11}{|c|}{ Control Variants } \\
\hline $57,963,470$ & $A>G$ & 11 & c. $1117+4 A>G$ & $3^{\prime}$ splice junction & $\mathrm{P}$ & M & $\mathrm{n} / \mathrm{a}$ & $\mathrm{n} / \mathrm{a}$ & $\mathrm{n} / \mathrm{a}$ & $\mathrm{n} / \mathrm{a}$ \\
\hline $57,966,423$ & $\mathrm{C}>\mathrm{T}$ & 15 & c. $1630 \mathrm{C}>\mathrm{T}$ & p.Arg544* & - & $\mathrm{F}$ & $\mathrm{n} / \mathrm{a}$ & $\mathrm{n} / \mathrm{a}$ & $\mathrm{n} / \mathrm{a}$ & $\mathrm{n} / \mathrm{a}$ \\
\hline $57,976,884$ & $\mathrm{G}>\mathrm{C}$ & 28 & c. $3021 \mathrm{G}>\mathrm{C}$ & $5^{\prime}$ splice junction & $\mathrm{N}$ & $\mathrm{F}$ & $\mathrm{n} / \mathrm{a}$ & $\mathrm{n} / \mathrm{a}$ & $\mathrm{n} / \mathrm{a}$ & $\mathrm{n} / \mathrm{a}$ \\
\hline \multicolumn{11}{|c|}{ FALS Variants } \\
\hline $57,975,729$ & $\mathrm{GA}>\mathrm{A}$ & 26 & c. $2987 \mathrm{del} A$ & p.Asp996fs & - & $M$ & 45 & $\mathrm{n} / \mathrm{a}$ & $n / a$ & $\mathrm{n} / \mathrm{a}$ \\
\hline $57,976,382$ & $\mathrm{C}>\mathrm{T}$ & 27 & c. $2993-3 C>T$ & $5^{\prime}$ splice junction & $\mathrm{Y}$ & M & 29 & L & $>264$ & $\mathrm{Y}$ \\
\hline $57,976,385$ & $\mathrm{GA}>\mathrm{G}$ & 27 & c. $2996 \mathrm{del} A$ & p.Asn999fs & - & M & 42 & L & $>12$ & Y \\
\hline $57,976,411$ & $A>G$ & 27 & c. $3019 A>G$ & p.Arg1007Gly & $\mathrm{Y}$ & $\mathrm{F}$ & 53 & L & 45 & $\mathrm{~N}$ \\
\hline $57,976,412$ & $\mathrm{G}>\mathrm{A}$ & 27 & c. $3020 \mathrm{G}>\mathrm{A}$ & p.Arg1007Lys & Y & M & 50 & L & $>108$ & Y \\
\hline $57,976,412$ & $\mathrm{G}>\mathrm{A}$ & 27 & c. $3020 \mathrm{G}>\mathrm{A}$ & p.Arg1007Lys & Y & $\mathrm{F}$ & 50 & $\mathrm{n} / \mathrm{a}$ & $>240$ & Y \\
\hline $57,976,413$ & $\mathrm{G}>\mathrm{A}$ & 27 & c. $3020+1 \mathrm{G}>\mathrm{A}$ & $3^{\prime}$ splice junction & Y & M & 45 & B & $>220$ & Y \\
\hline $57,976,414$ & $\mathrm{~T}>\mathrm{A}$ & 27 & c. $3020+2 T>A$ & $3^{\prime}$ splice junction & Y & M & 46 & B & 124 & $\mathrm{~N}$ \\
\hline $57,976,415$ & $A>G$ & 27 & c. $3020+3 A>G$ & $3^{\prime}$ splice junction & $Y$ & $\mathrm{M}$ & 50 & B & 54 & $\mathrm{~N}$ \\
\hline \multicolumn{11}{|c|}{ SALS Variants } \\
\hline $57,957,481$ & $\mathrm{G}>\mathrm{A}$ & 3 & c. $291+5 G>A$ & $3^{\prime}$ splice junction & $\mathrm{N}$ & $n / a$ & $n / a$ & $n / a$ & $n / a$ & $n / a$ \\
\hline $57,975,731$ & $\mathrm{CA}>\mathrm{C}$ & 26 & c.2989delA & p.Asn997fs & - & $\mathrm{F}$ & 50 & L & $>96$ & Y \\
\hline $57,976,384$ & $\mathrm{G}>\mathrm{A}$ & 27 & c. $2993-1 \mathrm{G}>\mathrm{A}$ & $5^{\prime}$ splice junction & $\mathrm{Y}$ & $\mathrm{n} / \mathrm{a}$ & 52 & B & $\mathrm{n} / \mathrm{a}$ & $\mathrm{n} / \mathrm{a}$ \\
\hline
\end{tabular}

P, possible; $Y$, yes; N, no; $M$, male; F, female; L, limb onset; B, bulbar onset, $n / a$, not available or applicable. Note that ASSEDA does not predict exon skipping based on frameshifts or nonsense mutations (Tompson et al., 2007). 


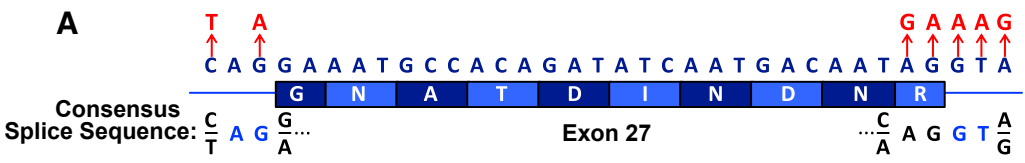

\section{C}

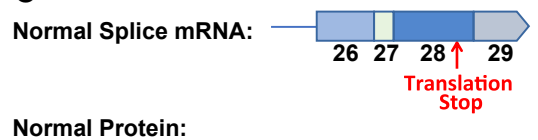

MDNGNATDINDNRSDLPCGYEAEDQAKLFPLHQETAAS*

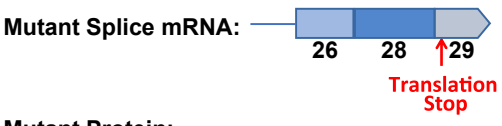

Mutant Protein:

MDNGVTCRVAMRLRTRPSFSLSTKRQQPANLPHPRLHTCTFSF*

D
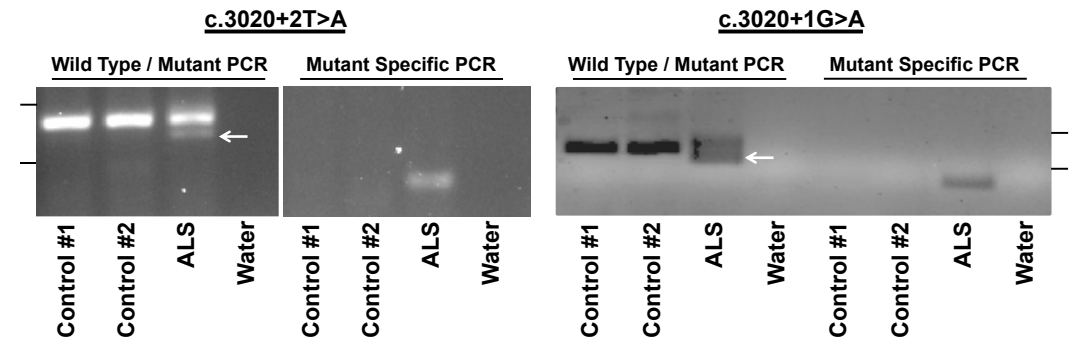

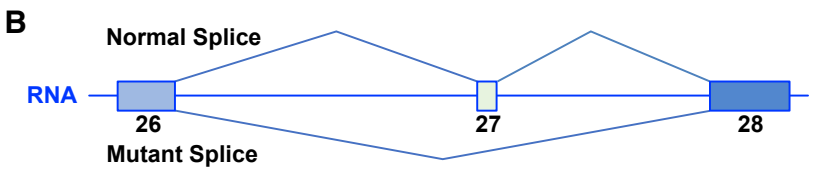

Figure 4. ALS-Associated LOF Variants of KIF5A Disrupt C-Terminal Sequence by Inducing Skipping of Exon 27

(A) Single nucleotide variants (SNVs) within KIF5A identified in ALS patients are clustered at the $5^{\prime}$ and $3^{\prime}$ splice junctions of exon 27 . The consensus splice sequence is shown.

(B) ALS-associated SNVs are predicted to induce skipping of exon 27 and result in an aberrant mRNA transcript.

(C) The skipping of exon 27 of KIF5A yields an outof-frame and extended disrupted C-terminal peptide sequence. The amino acids in red signify the divergence from the normal protein.

(D) RT-PCR was performed using RNA derived from ALS patients with the indicated LOF variant or without (controls) using primers to amplify either both wild-type (155 bp) and mutant (127 bp) splice forms or specifically the mutant splice form ( $80 \mathrm{bp}$, right panel). The arrow represents the position of the mutant-specific product. The tick marks represent $200 \mathrm{bp}$ (upper) and $100 \mathrm{bp}$ (lower) markers.

a relatively common, but low penetrance, risk allele for ALS, while LOF variants constitute rare, but high penetrance, risk factors.

patients with uncomplicated types of hereditary spastic paraparesis and CMT2 display a normal life expectancy (Patzkó and Shy, 2011).

\section{DISCUSSION}

We previously identified KIF5A as a candidate gene for ALS in our prior study that lacked the power to draw a definitive conclusion (Kenna et al., 2016). KIF5A was also a candidate ALS gene in a previous GWAS, though it similarly failed to reach genomewide significance (McLaughlin et al., 2017; van Rheenen et al., 2016), as well as a single gene study selected based on the a priori knowledge of its role in HSP/CMT2 and cytoskeletal function (Brenner et al., 2018). Here, we have confirmed KIF5A as an ALS-associated gene with genome-wide significance through two independent approaches. By performing a GWAS involving $\sim 80,000$ samples, in addition to replicating five previously published loci, as well as the previously reported locus SCFD1 using a linear mixed model analysis (data not shown), we identified a missense variant within the KIF5A gene that reached genomewide significance for association with ALS risk. It should be stated though, that as with all GWASs, we cannot rule out that other variants in linkage disequilibrium represent the primary risk factor. In an independent line of investigation, we applied RVB to exome sequencing of $\sim 21,000$ samples and identified an exome-wide significant association between FALS risk and rare KIF5A LOF variants. Analyses of KIF5A in independent replication cohorts both confirmed our initial finding for the p.Pro986Leu variant and revealed three additional carriers of LOF variants in 9,046 ALS cases. Taken together, our results indicate that the p.Pro986Leu KIF5A variant may represent
Kinesins are microtubule-based motor proteins involved in intracellular transport of organelles within eukaryotic cells. In mammals, there are three heavy-chain isoforms of KIF5: KIF5A, KIF5B, and KIF5C (Miki et al., 2001). The three proteins homo- and heterodimerize through their coiled-coiled stalk domain, and create a complex with two kinesin light chains via binding to the tail domain (Hirokawa et al., 1989). All three KIF5 genes are expressed in neurons (Kanai et al., 2000) and function to transport many cargos by binding to distinct adaptor proteins.

The central role of kinesins in axonal transport leads us to speculate that mutations in KIF5A cause disease by disrupting this process. Indeed, defects in axonal transport are a common observation in ALS patients and are already known to directly contribute to motor neuron degeneration pathogenesis (Chevalier-Larsen and Holzbaur, 2006; Hirokawa et al., 2010; Millecamps and Julien, 2013). KIF5 mediates the transport of granules containing both RNA and RNA-binding proteins within neuronal dendrites and axons (Kanai et al., 2004). Among these cargos are the ALS-associated proteins FUS and hnRNPA1 (Guo et al., 2017; Kim et al., 2013; Kwiatkowski et al., 2009; Vance et al., 2009). Similarly, KIF5 mediates the transport of VAPB through the adaptor protein protrudin (Matsuzaki et al., 2011), and mutations in the VAPB gene have been identified in ALS and late-onset spinal muscular atrophy (Nishimura et al., 2004, 2005). KIF5 is responsible for the axonal transport of neurofilaments (Wang and Brown, 2010) and KIF5A knockout mice display abnormal transport of neurofilaments (Xia et al., 2003). Abnormal accumulation of neurofilaments is a pathological hallmark of ALS and rare mutations in neurofilament heavy polypeptide (NEFH) are associated with ALS (Al-Chalabi et al., 1999). 


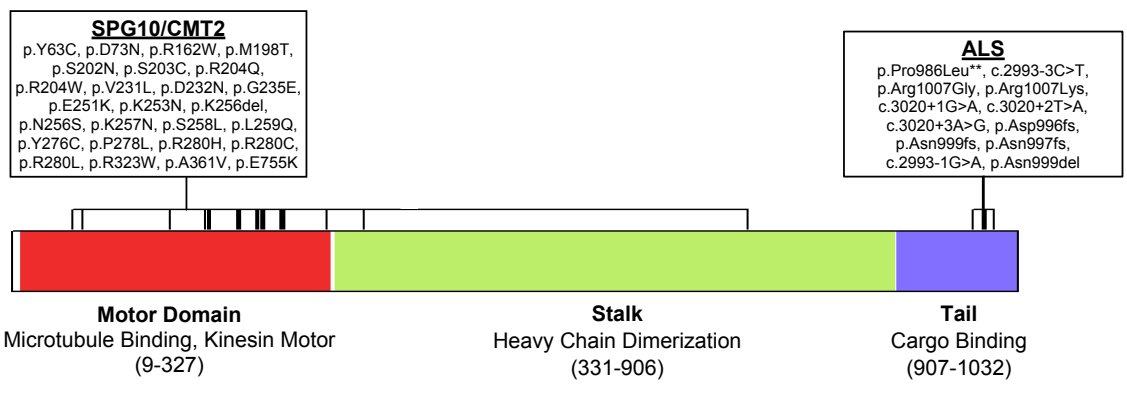

KIF5 also contributes to the transport of mitochondria (Kanai et al., 2000; Tanaka et al., 1998) and motor neurons derived from $K I F 5 A^{-1-}$ mice display transport deficits and reduced survival (Karle et al., 2012). Impaired transport and dysfunction of mitochondria represent another common hallmark observed in ALS patients (Chevalier-Larsen and Holzbaur, 2006; Guo et al., 2017; Palomo and Manfredi, 2015; Smith et al., 2017). KIF5 also contributes to the transport of AMPA-type (Heisler et al., 2014; Setou et al., 2002) and $\mathrm{GABA}_{A}$ receptors (Nakajima et al., 2012). In keeping with reported ALS genes such as NEK1 (Thiel et al., 2011) and PFN1 (Wu et al., 2012), modulation of KIF5A expression has been shown to influence the formation of neurite-like membrane protrusions (Matsuzaki et al., 2011). Given its critical interactions with the cytoskeleton, the identification of KIF5A mutations further extends the list of cytoskeletalrelated proteins implicated in ALS pathogenesis, such as PFN1, TUBA4A, NEFH, and peripherin (Al-Chalabi et al., 1999; Gros-Louis et al., 2004; Smith et al., 2014; Wu et al., 2012).

An important question raised by the current study is why variation within the $\mathrm{C}$-terminal cargo-binding domain is associated with ALS, while missense variations of the $\mathrm{N}$-terminal motor domain are associated with hereditary spastic paraparesis and CMT2. Missense mutations within this latter domain have been shown to affect microtubule binding and/or ATP hydrolysis, resulting in a defective KIF5A-mediated anterograde transport of cargo along dendrites and axons. This, in turn, leads to the axonal retrograde degeneration observed both in hereditary spastic paraparesis and CMT2, two length-dependent axonopathies (Ebbing et al., 2008). In contrast, the primary cellular lesion in ALS is believed to occur within motor neuron cell bodies, where cytoplasmic protein aggregates are consistently observed, and to propagate anterogradely along neurites. We anticipate that LOF variants within the C-terminal domain of KIF5A will disrupt binding with specific cargo proteins. This is supported by a study in zebrafish in which truncation of the $C$ terminus resulted in a dramatic disruption of axonal localization of mitochondria (Campbell et al., 2014). One possible mechanism is that disruption of binding to cargo may possibly lead to their accumulation and seed aggregation within the cell body, resulting in a deficiency at neurite terminals. Deficiency in KIF5A expression and cargo binding has been associated with accumulation of phosphorylated neurofilaments and amyloid precursor protein within neuronal cell bodies, and subsequent neurodegeneration, in patients with multiple sclerosis (Hares
Figure 5. KIF5A ALS Mutations Show Distinct Localization from Missense Mutations Previously Associated with SPG10 and CMT2

Causative mutations for SPG10 and CMT2 described within the literature (Crimella et al., 2012; Jennings et al., 2017; Liu et al., 2014; Reid et al., 2002) and ALS-associated mutations identified within this study are shown. As illustrated, mutations causative for SPG10/CMT2 are predominantly missense changes located in the $\mathrm{N}$-terminal motor domain. In contrast, ALS mutations are primarily located at the $\mathrm{C}$-terminal motor domain and are LOF. The double asterisk denotes the risk variant identified through the GWAS. et al., 2017). While differences in KIF5A kinetics and KIF5A interactions constitute one possibility to explain the phenotypic heterogeneity, it is also possible $\mathrm{C}$-terminal and $\mathrm{N}$-terminal variants act through a common mechanism, but that a difference in the relative extent of loss- or gain-of-function toxicities leads to milder (i.e., hereditary spastic paraplegia or CMT2) or more severe (i.e., ALS) phenotypes.

\section{STAR $\star M E T H O D S$}

Detailed methods are provided in the online version of this paper and include the following:

- KEY RESOURCES TABLE

- CONTACT FOR REAGENT AND RESOURCE SHARING

- EXPERIMENTAL MODEL AND SUBJECT DETAILS Study cohorts

- METHOD DETAILS

D Data generation and pre-processing

o Functional annotation of variants identified by WXS/WGS

RT-PCR Analysis

- QUANTIFICATION AND STATISTICAL ANALYSIS

O Statistical analyses

- DATA AND SOFTWARE AVAILABILITY

- Datasets

O Data Resources and Databases

O Software

\section{SUPPLEMENTAL INFORMATION}

Supplemental Information includes eight figures, eight tables, and consortia memberships and can be found with this article online at https://doi.org/10. 1016/j.neuron.2018.02.027.

\section{CONSORTIA}

The members of the ALS Sequencing Consortium are Andrew S. Allen, Stanley Appel, Robert H. Baloh, Richard S. Bedlack, Braden E. Boone, Robert Brown, John P. Carulli, Alessandra Chesi, Wendy K. Chung, Elizabeth T. Cirulli, Gregory M. Cooper, Julien Couthouis, Aaron G. Day-Williams, Patrick A. Dion, Summer Gibson, Aaron D. Gitler, Jonathan D. Glass, David B. Goldstein, Yujun Han, Matthew B. Harms, Tim Harris, Sebastian D. Hayes, Angela L. Jones, Jonathan Keebler, Brian J. Krueger, Brittany N. Lasseigne, Shawn E. Levy, Yi-Fan Lu, Tom Maniatis, Diane McKenna-Yasek, Timothy M. Miller, Richard M. Myers, Slavé Petrovski, Stefan M. Pulst, Alya R. Raphael, John M. Ravits, 
Zhong Ren, Guy A. Rouleau, Peter C. Sapp, Neil A. Shneider, Ericka Simpson, Katherine B. Sims, John F. Staropoli, Lindsay L. Waite, Quanli Wang, Jack R. Wimbish, and Winnie W. Xin.

The members of the Answer ALS Foundation are Julia Kaye, Steven Finkbeiner, Stacia Wyman, Alexander LeNail, Leandro Lima, Ernest Fraenkel, Jeffrey D. Rothstein, Clive N. Svendsen, Leslie M. Thompson, Jenny Van Eyk, Nicholas J. Maragakis, James D. Berry, Jonathan D. Glass, Timothy M. Miller, Stephen J. Kolb, Robert H. Baloh, Merit Cudkowicz, and Emily Baxi.

The members of the Clinical Research in ALS and Related Disorders for Therapeutic Development (CReATe) Consortium are Michael Benatar, J. Paul Taylor, Gang Wu, Evadnie Rampersaud, Joanne Wuu, Rosa Rademakers, Stephan Züchner, Rebecca Schule, Jacob McCauley, Sumaira Hussain, Anne Cooley, Marielle Wallace, Christine Clayman, Richard Barohn, Jeffrey Statland, John Ravits, Andrea Swenson, Carlayne Jackson, Jaya Trivedi, Shaida Khan, Jonathan Katz, Liberty Jenkins, Ted Burns, Kelly Gwathmey, James Caress, Corey McMillan, Lauren Elman, Erik Pioro, Jeannine Heckmann, Yuen So, David Walk, Samuel Maiser, and Jinghui Zhang.

The members of the French ALS Consortium are William Camu, Kevin Mouzat, Serge Lumbroso, Philippe Corcia, Vincent Meininger, Gérard Besson, Emmeline Lagrange, Pierre Clavelou, Nathalie Guy, Philippe Couratier, Patrick Vourch, Véronique Danel, Emilien Bernard, and Gwendal Lemasson.

The members of the Genomic Translation for ALS Care (GTAC) Consortium are Matthew B. Harms, David B. Goldstein, Neil A. Shneider, Stephen Goutman, Zachary Simmons, Timothy M. Miller, Siddharthan Chandran, Suvankar Pal, George Manousakis, Stanley H. Appel, Ericka Simpson, Leo Wang, Robert H. Baloh, Summer Gibson, Richard Bedlack, David Lacomis, Dhruv Sareen, Alexander Sherman, Lucie Bruijn, and Michelle Penny.

The members of the ITALSGEN Consortium are Francesco O. Logullo, Isabella Simone, Giancarlo Logroscino, Fabrizio Salvi, Ilaria Bartolomei, Giuseppe Borghero, Maria Rita Murru, Emanuela Costantino, Carla Pani, Roberta Puddu, Carla Caredda, Valeria Piras, Stefania Tranquilli, Stefania Cuccu, Daniela Corongiu, Maurizio Melis, Antonio Milia, Francesco Marrosu, Maria Giovanna Marrosu, Gianluca Floris, Antonino Cannas, Stefania Tranquilli, Margherita Capasso, Claudia Caponnetto, Gianluigi Mancardi, Paola Origone, Paola Mandich, Francesca L. Conforti, Sebastiano Cavallaro, Gabriele Mora, Kalliopi Marinou, Riccardo Sideri, Silvana Penco, Lorena Mosca, Christian Lunetta, Giuseppe Lauria Pinter, Massimo Corbo, Nilo Riva, Paola Carrera, Paolo Volanti, Jessica Mandrioli, Nicola Fini, Antonio Fasano, Lucio Tremolizzo, Alessandro Arosio, Carlo Ferrarese, Francesca Trojsi, Gioacchino Tedeschi, Maria Rosaria Monsurrò, Giovanni Piccirillo, Cinzia Femiano, Anna Ticca, Enzo Ortu, Vincenzo La Bella, Rossella Spataro, Tiziana Colletti, Mario Sabatelli, Marcella Zollino, Amelia Conte, Marco Luigetti, Serena Lattante, Giuseppe Marangi, Marialuisa Santarelli, Antonio Petrucci, Maura Pugliatti, Angelo Pirisi, Leslie D. Parish, Patrizia Occhineri, Fabio Giannini, Stefania Battistini, Claudia Ricci, Michele Benigni, Tea B. Cau, Daniela Loi, Andrea Calvo, Cristina Moglia, Maura Brunetti, Marco Barberis, Gabriella Restagno, Federico Casale, Giuseppe Marrali, Giuseppe Fuda, Irene Ossola, Stefania Cammarosano, Antonio Canosa, Antonio llardi, Umberto Manera, Maurizio Grassano, Raffaella Tanel, and Fabrizio Pisano.

The members of the NYGC ALS Consortium are Hemali Phatnani, Justin Kwan, Dhruv Sareen, James R. Broach, Zachary Simmons, Ximena ArcilaLondono, Edward B. Lee, Vivianna M. Van Deerlin, Neil A. Shneider, Ernest Fraenkel, Lyle W. Ostrow, Frank Baas, Noah Zaitlen, James D. Berry, Andrea Malaspina, Pietro Fratta, Gregory A. Cox, Leslie M. Thompson, Steve Finkbeiner, Efthimios Dardiotis, Timothy M. Miller, Siddharthan Chandran, Suvankar Pal, Eran Hornstein, Daniel J. MacGowan, Terry Heiman-Patterson, Molly G. Hammell, Nikolaos A. Patsopoulos, Joshua Dubnau, and Avindra Nath.

The members of the Project MinE ALS Sequencing Consortium are Ahmad Al Kheifat, Ammar Al-Chalabi, Peter Andersen, A. Nazli Basak, Ian P. Blair, Adriano Chio, Jonathan Cooper-Knock, Philippe Corcia, Philippe Couratier, Mamede de Carvalho, Annelot Dekker, Vivian Drory, Alberto Garcia Redondo, Marc Gotkine, Orla Hardiman, Winston Hide, Alfredo lacoangeli, Jonathan Glass, Kevin Kenna, Matthew Kiernan, Maarten Kooyman, John Landers, Russell McLaughlin, Bas Middelkoop, Jonathan Mill, Miguel Mitne Neto, Mattieu Moisse, Jesus Mora Pardina, Karen Morrison, Stephen Newhouse, Susana Pinto, Sara Pulit, Wim Robberecht, Aleksey Shatunov, Pamela Shaw, Chris Shaw, Vincenzo Silani, William Sproviero, Gijs Tazelaar, Nicola Ticozzi, Philip van Damme, Leonard van den Berg, Rick van der Spek, Kristel van Eijk, Michael van Es, Wouter van Rheenen, Joke van Vugt, Jan Veldink, Markus Weber, Kelly L. Williams, Mayana Zatz, Denis C. Bauer, and Natalie A. Twine.

The members of the SLAGEN Consortium are Vincenzo Silani, Nicola Ticozzi, Cinzia Gellera, Antonia Ratti, Franco Taroni, Giuseppe Lauria, Federico Verde, Isabella Fogh, Cinzia Tiloca, Giacomo P. Comi, Gianni Sorarù, Cristina Cereda, Sandra D'Alfonso, Lucia Corrado, Fabiola De Marchi, Stefania Corti, Mauro Ceroni, Letizia Mazzini, Gabriele Siciliano, Massimiliano Filosto, Maurizio Inghilleri, Silvia Peverelli, Claudia Colombrita, Barbara Poletti, Luca Maderna, Roberto Del Bo, Stella Gagliardi, Giorgia Querin, Cinzia Bertolin, Viviana Pensato, and Barbara Castellotti.

\section{ACKNOWLEDGMENTS}

The ALS Association (ALSA) provided funding support to Project MinE (15LGCA-235), the NYGC ALS Consortium (15-LGCA-234), the CReATe Consortium (17-LGCA-331), the GTAC Consortium (16-LGCA-310), the Target ALS Human Postmortem Tissue Core (16-LGCA-308), and NeuroLINCS, an $\mathrm{NIH}$-funded collaborative effort. P.V.D. is a senior investigator of FWO-Vlaanderen. Project MinE Belgium has been supported by ALS liga België, Flanders Innovation \& Enterpreneurship (IWT grant Project MinE), the Belgian National Lottery, and a grant from Opening the Future Fund (KU Leuven). W.R. is supported through the E. von Behring Chair for Neuromuscular and Neurodegenerative Disorders and ERC (grant agreement no. 340429). Additional funding support includes NINDS R35 NS097261 (R.R.) and P01NS084974 (R.R. and K.B.B.). A.N.B. thanks the Suna and Inan Kirac Foundation, Istanbul, TR for its generous support of the Neurodegeneration Research Laboratory throughout this study. Funding for this work was provided by the Heaton-Ellis Trust, the Middlemass Family, Motor Neurone Disease Association, Medical Research Council, Medical Research Foundation, the Psychiatry Research Trust of the Institute of Psychiatry, Guy's and St Thomas' Charity, the Wellcome Trust, and the Noreen Murray Foundation (C.E.S.). This work was also supported by the UK Dementia Research Institute, which is funded by the Medical Research Council, Alzheimer's Society, and Alzheimer's Research UK (C.E.S.). The salary for B.N.S. was funded by the Medical Research Foundation (MRF) (MRF-060-0003-RG-SMITH). P.C.S. was supported through the auspices of Dr. H. Robert Horvitz (Massachusetts Institute of Technology), an Investigator of the Howard Hughes Institute. Support for this work came from the Department of Veterans Affairs and NIH (P30AG13846) to N.W.K. I.P.B. is supported by the Motor Neurone Disease Research Institute of Australia and the National Health and Medical Research Council of Australia (1107644 and 1095215). P.F. is supported by an MRC/MNDA LEWF and by NIHR UCLH BRC. Research support from NIH/NIEHS (K23ES027221), the ALS Association, Target ALS, and Cytokinetics was provided to S.A.G. M. Cudkowicz was awarded funding from ALS Finding a Cure. N.T., C. Tiloca, C.G., V.S., and J.E.L. received research support from AriSLA - Fondazione Italiana di Ricerca per la SLA (grants EXOMEFALS and NOVALS) and the Italian Ministry of Health (grant GR-2011-02347820 - IRisALS). R.L. McLaughlin was supported by Science Foundation Ireland and the MND Association of England, Wales and Northern Ireland. O.H. is funded by the Health Research Board Clinician Scientist Programme and Science Foundation Ireland. P.J.S. is supported as an NIHR Senior Investigator (NF-SI-0512-10082). P.J.S. and J. Kirby are supported by the Sheffield NIHR Biomedical Research Centre for Translational Neuroscience (IS-BRC-1215-20017). A. Chiò receives research support from the Italian Ministry of Health (Ricerca Finalizzata), Regione Piemonte (Ricerca Finalizzata), University of Turin, Fondazione Vialli e Mauro onlus, and the European Commission (Health Seventh Framework Programme). P.M.A. is supported by research grants from the Swedish Brain Foundation, the Swedish Science Council, the Knut and Alice Wallenberg Foundation, the Bertil Hållsten Foundation, the Ulla-Carin Lindquist Foundation, the Neuroförbundet Association, the Torsten and Ragnar Söderberg Foundation, the Stratneuro Initiative, and Västerbotten County Council. R.B. received funding support from NINDS/ NS061867 and Target ALS. R.H.B.J. received funding from the Angel Fund, Project ALS/P2ALS, and the ALS Therapy Alliance. E. Rogaeva received funding support from the Canadian Consortium on Neurodegeneration in Aging. L. Myllykangas received funding support from Helsinki University Hospital and the Academy of Finland (grant 294817). P.T. received funding support from 
Helsinki University Hospital and the Sigrid Jusélius Foundation. J.D.G. received funding support from the ALS Association and Muscular Dystrophy Association. Additional funding was provided by the NIH/NINDS (R01NS073873, J.E.L.), the ALS Association (N.T., V.S., C.E.S., R.H.B.J., and J.E.L.), and the MND Association (N.T., V.S., C.E.S., and J.E.L.). J. Kaye, S.F., S.K.W., A.L., E.F., C.N.S., L.M.T., J.E.V.E., and J.D.R. received funding through NeuroLINCS (NIH U54 NS091046). The sequencing activities at NYGC were additionally supported by the TOW Foundation. The CReATe consortium (U54NS092091) is part of the Rare Diseases Clinical Research Network (RDCRN), an initiative of the Office of Rare Diseases Research (ORDR), NCATS. This consortium is funded through collaboration between NCATS and the NINDS. The Target ALS Human Postmortem Tissue Core received funding support from Target ALS (grant 90072272). The InCHIANTI study baseline (1998-2000) was supported as a "targeted project" (ICS110.1/RF97.71) by the Italian Ministry of Health and in part by the United States National Institute on Aging (contracts 263 MD 9164 and 263 MD 821336), the InCHIANTI follow-up 1 (2001-2003) was funded by the United States National Institute on Aging (contracts N.1-AG-1-1 and N.1-AG-12111), and the InCHIANTI follow-ups 2 and 3 studies (2004-2010) were financed by the United States National Institute on Aging (contract N01-AG5-0002). This work was supported in part by the Intramural Research Programs of the NIH, National Institute on Aging (Z01-AG000949-02); by the National Institute of Neurological Disorders and Stroke; and by Merck \& Co., Inc.. The work was also supported by the Center for Disease Control and Prevention, the Muscular Dystrophy Association, Microsoft Research, the Packard Center for ALS Research at Johns Hopkins, the ALS Association, UK MND Association, Medical Research Council (MRC) UK, Wellcome Trust/MRC Joint Call in Neurodegeneration Award, MRC Neuromuscular Centre, UK National Institute for Health Research Biomedical Research Unit, Italian Health Ministry (Ricerca Sanitaria Finalizzata 2007), Fondazione Vialli e Mauro Onlus, Compagnia di San Paolo, and European Community's Health Seventh Framework Programme (FP7/2007-2013) under grant agreement 259867. Role of the sponsors: the sponsors did not participate in the design and conduct of the study; collection, management, analysis, or interpretation of the data; preparation, review, or approval of the manuscript; or decision to submit the manuscript for publication. We thank the patients and research subjects who contributed samples for this study. This study used DNA samples and clinical data from the Target ALS Human Postmortem Tissue Core, the NINDS Repository at Coriell, the North East ALS (NEALS) Consortium Biorepository, the New York Brain Bank-The Taub Institute, Columbia University, Department of Veterans Affairs Biorepository Brain Bank (grant \#BX002466; C.B.B.), the Baltimore Longitudinal Study of Aging (BLSA) and the Johns Hopkins University Alzheimer's Disease Research Center, the NICHD Brain and Tissue Bank for Developmental Disorders at the University of Maryland, and the Australian MND DNA Bank. We also thank Crystal Pacut, Blake Swihart, and Jayna Duell, $\mathrm{RN}$ for assistance in study coordination (S.A.G.). This study utilized the highperformance computational capabilities of the Biowulf Linux cluster at the $\mathrm{NIH}$, Bethesda, Maryland (http://hpc.nih.gov), and the Massachusetts Green High Performance Computing Center at the University of Massachusetts Medical School (https://www.mghpcc.org). This study also used genotype and clinical data from the Wellcome Trust Case Control Consortium.

\section{AUTHOR CONTRIBUTIONS}

Sample Collection, Preparation, and Clinical Evaluation: N.T., B.J.K., P.K., W.v.R., J.J.F.A.v.V., R.A.V.d.S., B.N.S., G. Marangi, S.D.T., A.S.G., A. Kenna, ITALSGEN Consortium, ALS Sequencing Consortium, Answer ALS Foundation, G. Mora, A. Calvo, L. Mazzini, N.R., J.M., C. Caponnetto, S.B., P.V., V.L.B., F.L.C., G.B., S.M., I.L.S., F. Trojsi, F.S., F.O.L., S.D., L.C., M. Capasso, L.F., Genomic Translation for ALS Care (GTAC) Consortium, C.d.A.M.M., S.K., D.B.G., A.D.G., T.H., R.M.M., NYGC ALS Consortium, H.P., R.L. Musunuri, U.S.E., A.A., M.C.Z., J. Kaye, S.F., S.K.W., A.L., L.L., E.F., C.N.S., L.M.T., J.E.V.E., J.D.B., T.M.M., S.J.K., M. Cudkowicz, E.B., Clinical Research in ALS and Related Disorders for Therapeutic Development (CReATe) Consortium, M.B., J.W., SLAGEN Consortium, G.L., F.V., I.F., C. Tiloca, G.P.C., G.S., French ALS Consortium, P.C., H.L., L. Myllykangas, L.J., M.V., J.E., H.H., S.R., S.P.-B., R.W.O., K.C.S., A.M., J.H., P.C.S., D.M.-Y., M.P., A.
King, C. Troakes, C.V., J.d.B., F.B., A.L.M.A.t.A., J.L.M.-B., S.W.S., M.K.F., F.L., R.B., S.M.P., J.M.R., D.J.L.M., J. Kirby, E.P.P., R.P., J.B., G.G., T.L.D., C.B.B., N.W.K., J.C.T., I.L.B., K.M., S.L., T.D.H.-P., F.K., L.V.D.B., R.H.B., T.M.S., T.M., A.S., K.R.V.E., M.d.C., M.K., B.M., M.M., R.L. McLaughlin, M.A.V.E., M.W., K.B.B., M.V.B., R.R., K.E.M., A.N.B., J.S.M., V.E.D., P.J.S., M.R.T., K.T., O.H., K.L.W., J.A.F., G.A.N., I.P.B., G.A.R., J.E.-P., A.G.-R., A.A.-C., Project MinE ALS Sequencing Consortium, E. Rogaeva, L.Z., L.W.O., N.J.M., J.D.R., Z.S., J.C.-K., A.B., S.A.G., E.L.F., S.B.G., F. Taroni, A.R., C.G., P.V.D., W.R., P.F., M.S., C.L., A.C.L., P.M.A., J.H.W., W.C. J.Q.T., V.M.V.D., R.H.B.J., L.H.v.d.B., J.H.V., M.B.H., J.D.G., D.J.S., P.T., V.S., A. Chiò, C.E.S., B.J.T., and J.E.L. Performed Experiments and Data Analysis: A.N., K.P.K., A.E.R., N.T., F.F., R.C., J.A.D., B.J.K., M.A.N., P.K., A.M.R., W.v.R., N.A.M., J.J.F.A.v.V., J.T.G., R.A.V.d.S., H.A.P., Shankaracharya, B.N.S., G. Marangi, S. Asress, S.A.-S., S.D.T., Y.A., A.S.G., J.D.E., A. Kenna, Genomic Translation for ALS Care (GTAC) Consortium, ALS Sequencing Consortium, Answer ALS Foundation, C.d.A.M.M., S.K., D.B.G., A.D.G., T.H. R.M.M., NYGC ALS Consortium, H.P., R.L. Musunuri, U.S.E., A.A., M.C.Z., J. Kaye, S.F., S.K.W., A.L., L.L., E.F., C.N.S., L.M.T., J.E.V.E., J.D.B., T.M.M., S.J.K., M. Cudkowicz, E.B., Clinical Research in ALS and Related Disorders for Therapeutic Development (CReATe) Consortium, E. Rampersaud G.W., SLAGEN Consortium, G.L., F.V., I.F., C. Tiloca, G.P.C., G.S., A.B.S., J.O.J., S. Arepalli, P.C.S., D.M.-Y., M.P., C. Troakes, C.V., J.d.B., F.B., A.L.M.A.t.A., J.L.M.-B., D.G.H., J.D., J.R.G., S.W.S., R.H.C., T.D.H.-P., L.V.D.B., R.H.B., T.M.S., T.M., A.S., K.R.V.E., M.d.C., M.K., B.M., M.M., R.L. McLaughlin, M.A.V.E., M.W., K.B.B., M.V.B., R.R., K.E.M., A.N.B., J.S.M., V.E.D., P.J.S., M.R.T., K.T., O.H., K.L.W., J.A.F., G.A.N., I.P.B., G.A.R., J.E., A.G.-R., A.A.-C., Project MinE ALS Sequencing Consortium, E. Rogaeva, L.W.O., N.J.M., J.D.R., Z.S., J.C.-K., S.A.G., E.L.F., F. Taroni, A.R., C.G., P.V.D., W.R., A.C.L., P.M.A., J.H.W., W.C., J.Q.T., V.M.V.D., R.H.B.J., L.H.v.d.B., J.H.V., M.B.H., J.D.G., D.J.S., P.T., V.S., A. Chiò, C.E.S., B.J.T., and J.E.L. Scientific Planning and Direction: A.N., K.P.K., A.E.R., N.T., F.F., R.C., J.A.D., B.J.K., M.A.N., P.K., A.M.R., W.v.R., N.A.M., J.J.F.A.v.V., J.T.G., ALS Sequencing Consortium, Answer ALS Foundation, R.A.V.d.S., C Cereda, H.A.P., Shankaracharya, B.N.S., G. Marangi, S.D.T., Y.A., J.D.E., ITALSGEN Consortium, G. Mora, A. Calvo, L. Mazzini, N.R., J.M., C. Caponnetto, S.B., P.V., V.L.B., F.L.C., G.B., S.M., I.L.S., F. Trojsi, F.S., F.O.L., S.D., L.C., M. Capasso, L.F., Genomic Translation for ALS Care (GTAC) Consortium, D.B.G., A.D.G., T.H., R.M.M., NYGC ALS Consortium, H.P., J. Kaye, S.F. S.K.W., A.L., L.L., E.F., C.N.S., L.M.T., J.E.V.E., J.D.B., T.M.M., S.J.K., M. Cudkowicz, E.B., Clinical Research in ALS and Related Disorders for Therapeutic Development (CReATe) Consortium, M.B., J.P.T., J.W., French ALS Consortium, P.C., H.L., L. Myllykangas, L.J., M.V., J.E., H.H., S.R., S.P.-B., R.W.O., K.C.S., A.M., J.H., A.B.S., C. Troakes, C.V., J.d.B., F.B., A.L.M.A.t.A., J.L.M.-B., D.G.H., J.R.G., S.W.S., M.K.F., F.L., R.B., S.M.P., J.M.R., D.J.L.M., J. Kirby, E.P.P., R.P., J.B., G.G., T.L.D., C.B.B., N.W.K., J.C.T., I.L.B., K.M., S.L., T.D.H.-P., F.K., L.V.D.B., R.H.B., T.M.S., T.M., A.S., K.R.V.E., M.d.C., M.K., B.M., M.M., R.L. McLaughlin, M.A.V.E., M.W., K.B.B., M.V.B., R.R., K.E.M., A.N.B., J.S.M., V.E.D., P.J.S., M.R.T., K.T., O.H., K.L.W., J.A.F., G.A.N., I.P.B., G.A.R., J.E., A.G.-R., A.A.-C., Project MinE ALS Sequencing Consortium, E. Rogaeva, L.Z., L.W.O., N.J.M., J.D.R., Z.S., J.C.-K., A.B., S.A.G., E.L.F., S.B.G., F. Taroni, A.R., C.G., P.V.D., W.R., P.F., M.S., C.L., A.C.L., P.M.A., J.H.W., W.C., J.Q.T., V.M.V.D., R.H.B.J., L.H.v.d.B., J.H.V., M.B.H., J.D.G., D.J.S., P.T., V.S., A. Chiò, C.E.S., B.J.T., and J.E.L. Initia Manuscript Preparation: A.N., K.P.K., A.E.R., N.T., F.F., R.C., J.A.D., M.A.N. A.B.S., S.W.S., J.H.V., D.J.S., P.T., V.S., A. Chiò, C.E.S., B.J.T., and J.E.L.

\section{DECLARATION OF INTERESTS}

J.D.B. is a consultant to Neuraltus Pharmaceuticals and Denali Therapeutics, and held a research fellow position funded by Voyager Therapeutics. M. Cudkowicz has been a consultant for Eli Lilly and Company, Mitsubishi Tanabe Pharma America (MT Pharma America), Denali Therapeutics, Karyopharm Therapeutics, and Cytokinetics. S.A.G. has served as a consultant for and received research support from Cytokinetics. O.H. has received speaking honoraria from Novartis, Biogen Idec, Sanofi Aventis, and Merck-Serono and has been a member of advisory panels for Biogen Idec, Allergen, Ono Pharmaceuticals, Novartis, Cytokinetics, and Sanofi Aventis. O.H. serves as 
Editor-in-Chief of Amyotrophic Lateral Sclerosis and Frontotemporal Dementia. L.H.v.d.B. serves on scientific advisory boards for the Prinses Beatrix Spierfonds, Thierry Latran Foundation, Biogen, and Cytokinetics and serves on the editorial board of Amyotrophic Lateral Sclerosis and Frontotemporal Degeneration and the Journal of Neurology, Neurosurgery, and Psychiatry. J.H.V. reports that his institute received consultancy fees from Vertex Pharmaceuticals. A. Chiò serves on scientific advisory boards for Biogen Idec, Cytokinetics, Italfarmaco, and Neuraltus. P.M.A. serves on advisory board panels for Biogen and Orphazyme. V.S. serves as a consultant for Cytokinetics.

Received: December 21, 2017

Revised: January 21, 2018

Accepted: February 26, 2018

Published: March 21, 2018

\section{REFERENCES}

Ahmeti, K.B., Ajroud-Driss, S., Al-Chalabi, A., Andersen, P.M., Armstrong, J., Birve, A., Blauw, H.M., Brown, R.H., Bruijn, L., Chen, W., et al.; ITALSGEN consortium; ALSGEN Consortium (2013). Age of onset of amyotrophic lateral sclerosis is modulated by a locus on 1p34.1. Neurobiol. Aging 34, 357.e7-357.e19.

Al-Chalabi, A., Andersen, P.M., Nilsson, P., Chioza, B., Andersson, J.L., Russ, C., Shaw, C.E., Powell, J.F., and Leigh, P.N. (1999). Deletions of the heavy neurofilament subunit tail in amyotrophic lateral sclerosis. Hum. Mol. Genet. 8, 157-164.

Arthur, K.C., Calvo, A., Price, T.R., Geiger, J.T., Chiò, A., and Traynor, B.J. (2016). Projected increase in amyotrophic lateral sclerosis from 2015 to 2040. Nat. Commun. 7, 12408.

Benyamin, B., He, J., Zhao, Q., Gratten, J., Garton, F., Leo, P.J., Liu, Z., Mangelsdorf, M., Al-Chalabi, A., Anderson, L., et al. (2017). Cross-ethnic meta-analysis identifies association of the GPX3-TNIP1 locus with amyotrophic lateral sclerosis. Nat. Commun. 8, 611.

Brenner, D., Yilmaz, R., Müller, K., Grehl, T., Petri, S., Meyer, T., Grosskreutz, J., Weydt, P., Ruf, W., Neuwirth, C., et al.; German ALS network MND-NET (2018). Hot-spot KIF5A mutations cause familial ALS. Brain. https://doi.org/ 10.1093/brain/awX370.

Brooks, B.R. (1994). El Escorial World Federation of Neurology criteria for the diagnosis of amyotrophic lateral sclerosis. Subcommittee on Motor Neuron Diseases/Amyotrophic Lateral Sclerosis of the World Federation of Neurology Research Group on Neuromuscular Diseases and the El Escorial "Clinical limits of amyotrophic lateral sclerosis" workshop contributors. J. Neurol. Sci. 124 (Suppl), 96-107.

Caminsky, N., Mucaki, E.J., and Rogan, P.K. (2014). Interpretation of mRNA splicing mutations in genetic disease: review of the literature and guidelines for information-theoretical analysis. F1000Res. 3, 282.

Campbell, P.D., Shen, K., Sapio, M.R., Glenn, T.D., Talbot, W.S., and Marlow, F.L. (2014). Unique function of Kinesin Kif5A in localization of mitochondria in axons. J. Neurosci. 34, 14717-14732.

Chang, C.C., Chow, C.C., Tellier, L.C., Vattikuti, S., Purcell, S.M., and Lee, J.J. (2015). Second-generation PLINK: rising to the challenge of larger and richer datasets. Gigascience 4, 7.

Chevalier-Larsen, E., and Holzbaur, E.L.F. (2006). Axonal transport and neurodegenerative disease. Biochim. Biophys. Acta 1762, 1094-1108.

Chia, R., Chiò, A., and Traynor, B.J. (2018). Novel genes associated with amyotrophic lateral sclerosis: diagnostic and clinical implications. Lancet Neurol. 17, 94-102.

Chiò, A., Logroscino, G., Hardiman, O., Swingler, R., Mitchell, D., Beghi, E., and Traynor, B.G.; Eurals Consortium (2009). Prognostic factors in ALS: a critical review. Amyotroph. Lateral Scler. 10, 310-323.

Cingolani, P., Platts, A., Wang, L., Coon, M., Nguyen, T., Wang, L., Land, S.J., Lu, X., and Ruden, D.M. (2012). A program for annotating and predicting the effects of single nucleotide polymorphisms, SnpEff: SNPs in the genome of Drosophila melanogaster strain w1118; iso-2; iso-3. Fly (Austin) 6, 80-92.
Cirulli, E.T., Lasseigne, B.N., Petrovski, S., Sapp, P.C., Dion, P.A., Leblond, C.S., Couthouis, J., Lu, Y.-F., Wang, Q., Krueger, B.J., et al.; FALS Sequencing Consortium (2015). Exome sequencing in amyotrophic lateral sclerosis identifies risk genes and pathways. Science 347, 1436-1441.

Coppedè, F., and Migliore, L. (2015). DNA damage in neurodegenerative diseases. Mutat. Res. 776, 84-97.

Crimella, C., Baschirotto, C., Arnoldi, A., Tonelli, A., Tenderini, E., Airoldi, G., Martinuzzi, A., Trabacca, A., Losito, L., Scarlato, M., et al. (2012). Mutations in the motor and stalk domains of KIF5A in spastic paraplegia type 10 and in axonal Charcot-Marie-Tooth type 2. Clin. Genet. 82, 157-164.

Das, S., Forer, L., Schönherr, S., Sidore, C., Locke, A.E., Kwong, A., Vrieze, S.I., Chew, E.Y., Levy, S., McGue, M., et al. (2016). Next-generation genotype imputation service and methods. Nat. Genet. 48, 1284-1287.

DeJesus-Hernandez, M., Mackenzie, I.R., Boeve, B.F., Boxer, A.L., Baker, M., Rutherford, N.J., Nicholson, A.M., Finch, N.A., Flynn, H., Adamson, J., et al. (2011). Expanded GGGGCC hexanucleotide repeat in noncoding region of C9ORF72 causes chromosome 9p-linked FTD and ALS. Neuron 72, 245-256.

Delaneau, O., Zagury, J.-F., and Marchini, J. (2013). Improved whole-chromosome phasing for disease and population genetic studies. Nat. Methods 10, 5-6.

Ebbing, B., Mann, K., Starosta, A., Jaud, J., Schöls, L., Schüle, R., and Woehlke, G. (2008). Effect of spastic paraplegia mutations in KIF5A kinesin on transport activity. Hum. Mol. Genet. 17, 1245-1252.

Fang, H., Wu, Y., Narzisi, G., O’Rawe, J.A., Barrón, L.T.J., Rosenbaum, J., Ronemus, M., lossifov, I., Schatz, M.C., and Lyon, G.J. (2014). Reducing INDEL calling errors in whole genome and exome sequencing data. Genome Med. 6, 89

Fang, X., Lin, H., Wang, X., Zuo, Q., Qin, J., and Zhang, P. (2015). The NEK1 interactor, C21ORF2, is required for efficient DNA damage repair. Acta Biochim. Biophys. Sin. (Shanghai) 47, 834-841.

Freischmidt, A., Wieland, T., Richter, B., Ruf, W., Schaeffer, V., Müller, K., Marroquin, N., Nordin, F., Hübers, A., Weydt, P., et al. (2015). Haploinsufficiency of TBK1 causes familial ALS and fronto-temporal dementia. Nat. Neurosci. 18, 631-636.

Gladman, M., and Zinman, L. (2015). The economic impact of amyotrophic lateral sclerosis: a systematic review. Expert Rev. Pharmacoecon. Outcomes Res. 15, 439-450.

Gros-Louis, F., Larivière, R., Gowing, G., Laurent, S., Camu, W., Bouchard, J.P., Meininger, V., Rouleau, G.A., and Julien, J.P. (2004). A frameshift deletion in peripherin gene associated with amyotrophic lateral sclerosis. J. Biol. Chem. $279,45951-45956$.

Guo, W., Naujock, M., Fumagalli, L., Vandoorne, T., Baatsen, P., Boon, R., Ordovás, L., Patel, A., Welters, M., Vanwelden, T., et al. (2017). HDAC6 inhibition reverses axonal transport defects in motor neurons derived from FUS-ALS patients. Nat. Commun. 8, 861 .

Hares, K., Redondo, J., Kemp, K., Rice, C., Scolding, N., and Wilkins, A. (2017). Axonal motor protein KIF5A and associated cargo deficits in multiple sclerosis lesional and normal-appearing white matter. Neuropathol. Appl. Neurobiol. 43, 227-241.

Heisler, F.F., Lee, H.K., Gromova, K.V., Pechmann, Y., Schurek, B., Ruschkies, L., Schroeder, M., Schweizer, M., and Kneussel, M. (2014). GRIP1 interlinks $\mathrm{N}$-cadherin and AMPA receptors at vesicles to promote combined cargo transport into dendrites. Proc. Natl. Acad. Sci. USA 111, 5030-5035.

Hirokawa, N., Pfister, K.K., Yorifuji, H., Wagner, M.C., Brady, S.T., and Bloom, G.S. (1989). Submolecular domains of bovine brain kinesin identified by electron microscopy and monoclonal antibody decoration. Cell 56, 867-878.

Hirokawa, N., Niwa, S., and Tanaka, Y. (2010). Molecular motors in neurons: transport mechanisms and roles in brain function, development, and disease. Neuron 68, 610-638.

Hirtz, D., Thurman, D.J., Gwinn-Hardy, K., Mohamed, M., Chaudhuri, A.R., and Zalutsky, R. (2007). How common are the "common" neurologic disorders? Neurology 68, 326-337. 
Jennings, S., Chenevert, M., Liu, L., Mottamal, M., Wojcik, E.J., and Huckaba, T.M. (2017). Characterization of kinesin switch I mutations that cause hereditary spastic paraplegia. PLoS ONE 12, e0180353.

Jian, X., Boerwinkle, E., and Liu, X. (2014). In silico prediction of splice-altering single nucleotide variants in the human genome. Nucleic Acids Res. 42, 13534-13544.

Kanai, Y., Okada, Y., Tanaka, Y., Harada, A., Terada, S., and Hirokawa, N. (2000). KIF5C, a novel neuronal kinesin enriched in motor neurons. J. Neurosci. 20, 6374-6384.

Kanai, Y., Dohmae, N., and Hirokawa, N. (2004). Kinesin transports RNA: isolation and characterization of an RNA-transporting granule. Neuron 43, 513-525.

Karle, K.N., Möckel, D., Reid, E., and Schöls, L. (2012). Axonal transport deficit in a $\operatorname{KIF5A(-/-)~mouse~model.~Neurogenetics~13,~169-179.~}$

Kenna, K.P., van Doormaal, P.T.C., Dekker, A.M., Ticozzi, N., Kenna, B.J., Diekstra, F.P., van Rheenen, W., van Eijk, K.R., Jones, A.R., Keagle, P., et al.; SLAGEN Consortium (2016). NEK1 variants confer susceptibility to amyotrophic lateral sclerosis. Nat. Genet. 48, 1037-1042.

Kim, H.J., Kim, N.C., Wang, Y.-D., Scarborough, E.A., Moore, J., Diaz, Z., MacLea, K.S., Freibaum, B., Li, S., Molliex, A., et al. (2013). Mutations in prion-like domains in hnRNPA2B1 and hnRNPA1 cause multisystem proteinopathy and ALS. Nature 495, 467-473.

Kwiatkowski, T.J., Jr., Bosco, D.A., Leclerc, A.L., Tamrazian, E., Vanderburg, C.R., Russ, C., Davis, A., Gilchrist, J., Kasarskis, E.J., Munsat, T., et al. (2009). Mutations in the FUS/TLS gene on chromosome 16 cause familial amyotrophic lateral sclerosis. Science 323, 1205-1208.

Laaksovirta, H., Peuralinna, T., Schymick, J.C., Scholz, S.W., Lai, S.-L., Myllykangas, L., Sulkava, R., Jansson, L., Hernandez, D.G., Gibbs, J.R., et al. (2010). Chromosome 9p21 in amyotrophic lateral sclerosis in Finland: a genome-wide association study. Lancet Neurol. 9, 978-985.

Liu, X., Jian, X., and Boerwinkle, E. (2013). dbNSFP v2.0: a database of human non-synonymous SNVs and their functional predictions and annotations. Hum. Mutat. 34, E2393-E2402.

Liu, Y.-T., Laurá, M., Hersheson, J., Horga, A., Jaunmuktane, Z., Brandner, S., Pittman, A., Hughes, D., Polke, J.M., Sweeney, M.G., et al. (2014). Extended phenotypic spectrum of KIF5A mutations: From spastic paraplegia to axonal neuropathy. Neurology 83, 612-619.

Lopez-Gonzalez, R., Lu, Y., Gendron, T.F., Karydas, A., Tran, H., Yang, D., Petrucelli, L., Miller, B.L., Almeida, S., and Gao, F.-B. (2016). Poly(GR) in C9ORF72-related ALS/FTD compromises mitochondrial function and increases oxidative stress and DNA damage in iPSC-derived motor neurons. Neuron 92, 383-391.

Madabhushi, R., Pan, L., and Tsai, L.-H. (2014). DNA damage and its links to neurodegeneration. Neuron 83, 266-282.

Majounie, E., Renton, A.E., Mok, K., Dopper, E.G.P., Waite, A., Rollinson, S., Chiò, A., Restagno, G., Nicolaou, N., Simón-Sánchez, J., et al.; Chromosome 9-ALS/FTD Consortium; French research network on FTLD/FTLD/ALS; ITALSGEN Consortium (2012). Frequency of the C9orf72 hexanucleotide repeat expansion in patients with amyotrophic lateral sclerosis and frontotemporal dementia: a cross-sectional study. Lancet Neurol. 11, 323-330.

Malovannaya, A., Lanz, R.B., Jung, S.Y., Bulynko, Y., Le, N.T., Chan, D.W., Ding, C., Shi, Y., Yucer, N., Krenciute, G., et al. (2011). Analysis of the human endogenous coregulator complexome. Cell 145, 787-799.

Manichaikul, A., Mychaleckyj, J.C., Rich, S.S., Daly, K., Sale, M., and Chen, W.-M. (2010). Robust relationship inference in genome-wide association studies. Bioinformatics 26, 2867-2873.

Marchini, J., and Howie, B. (2010). Genotype imputation for genome-wide association studies. Nat. Rev. Genet. 11, 499-511.

Maruyama, H., Morino, H., Ito, H., Izumi, Y., Kato, H., Watanabe, Y., Kinoshita, Y., Kamada, M., Nodera, H., Suzuki, H., et al. (2010). Mutations of optineurin in amyotrophic lateral sclerosis. Nature 465, 223-226.
Matsuzaki, F., Shirane, M., Matsumoto, M., and Nakayama, K.I. (2011). Protrudin serves as an adaptor molecule that connects KIF5 and its cargoes in vesicular transport during process formation. Mol. Biol. Cell 22, 4602-4620. McLaughlin, R.L., Schijven, D., van Rheenen, W., van Eijk, K.R., O’Brien, M., Kahn, R.S., Ophoff, R.A., Goris, A., Bradley, D.G., Al-Chalabi, A., et al.; Project MinE GWAS Consortium; Schizophrenia Working Group of the Psychiatric Genomics Consortium (2017). Genetic correlation between amyotrophic lateral sclerosis and schizophrenia. Nat. Commun. 8, 14774.

Miki, H., Setou, M., Kaneshiro, K., and Hirokawa, N. (2001). All kinesin superfamily protein, KIF, genes in mouse and human. Proc. Natl. Acad. Sci. USA 98, 7004-7011.

Millecamps, S., and Julien, J.-P. (2013). Axonal transport deficits and neurodegenerative diseases. Nat. Rev. Neurosci. 14, 161-176.

Morton, S., Hesson, L., Peggie, M., and Cohen, P. (2008). Enhanced binding of TBK1 by an optineurin mutant that causes a familial form of primary open angle glaucoma. FEBS Lett. 582, 997-1002.

Nakajima, K., Yin, X., Takei, Y., Seog, D.-H., Homma, N., and Hirokawa, N. (2012). Molecular motor KIF5A is essential for GABA(A) receptor transport, and KIF5A deletion causes epilepsy. Neuron 76, 945-961.

Nishimura, A.L., Mitne-Neto, M., Silva, H.C.A., Richieri-Costa, A., Middleton, S., Cascio, D., Kok, F., Oliveira, J.R.M., Gillingwater, T., Webb, J., et al. (2004). A mutation in the vesicle-trafficking protein VAPB causes late-onset spinal muscular atrophy and amyotrophic lateral sclerosis. Am. J. Hum. Genet. 75, 822-831.

Nishimura, A.L., Al-Chalabi, A., and Zatz, M. (2005). A common founder for amyotrophic lateral sclerosis type 8 (ALS8) in the Brazilian population. Hum. Genet. 118, 499-500.

Palomo, G.M., and Manfredi, G. (2015). Exploring new pathways of neurodegeneration in ALS: the role of mitochondria quality control. Brain Res. 1607, 36-46.

Patzkó, A., and Shy, M.E. (2011). Update on Charcot-Marie-Tooth disease. Curr. Neurol. Neurosci. Rep. 11, 78-88.

Pe'er, I., Yelensky, R., Altshuler, D., and Daly, M.J. (2008). Estimation of the multiple testing burden for genomewide association studies of nearly all common variants. Genet. Epidemiol. 32, 381-385.

Reid, E., Kloos, M., Ashley-Koch, A., Hughes, L., Bevan, S., Svenson, I.K., Graham, F.L., Gaskell, P.C., Dearlove, A., Pericak-Vance, M.A., et al. (2002). A kinesin heavy chain (KIF5A) mutation in hereditary spastic paraplegia (SPG10). Am. J. Hum. Genet. 71, 1189-1194.

Renton, A.E., Majounie, E., Waite, A., Simón-Sánchez, J., Rollinson, S., Gibbs, J.R., Schymick, J.C., Laaksovirta, H., van Swieten, J.C., Myllykangas, L., et al.; ITALSGEN Consortium (2011). A hexanucleotide repeat expansion in C9ORF72 is the cause of chromosome 9p21-linked ALS-FTD. Neuron 72, 257-268.

Renton, A.E., Chiò, A., and Traynor, B.J. (2014). State of play in amyotrophic lateral sclerosis genetics. Nat. Neurosci. 17, 17-23.

Robberecht, W., and Eykens, C. (2015). The genetic basis of amyotrophic lateral sclerosis. Adv. Genomics Genet. 5, 327-345.

Rosen, D.R., Siddique, T., Patterson, D., Figlewicz, D.A., Sapp, P., Hentati, A., Donaldson, D., Goto, J., O'Regan, J.P., Deng, H.X., et al. (1993). Mutations in $\mathrm{Cu} / \mathrm{Zn}$ superoxide dismutase gene are associated with familial amyotrophic lateral sclerosis. Nature 362, 59-62.

Salvi, E., Kutalik, Z., Glorioso, N., Benaglio, P., Frau, F., Kuznetsova, T., Arima, H., Hoggart, C., Tichet, J., Nikitin, Y.P., et al. (2012). Genomewide association study using a high-density single nucleotide polymorphism array and case-control design identifies a novel essential hypertension susceptibility locus in the promoter region of endothelial NO synthase. Hypertension 59 248-255.

Setou, M., Seog, D.-H., Tanaka, Y., Kanai, Y., Takei, Y., Kawagishi, M., and Hirokawa, N. (2002). Glutamate-receptor-interacting protein GRIP1 directly steers kinesin to dendrites. Nature 417, 83-87.

Shatunov, A., Mok, K., Newhouse, S., Weale, M.E., Smith, B., Vance, C. Johnson, L., Veldink, J.H., van Es, M.A., van den Berg, L.H., et al. (2010). 
Chromosome 9p21 in sporadic amyotrophic lateral sclerosis in the UK and seven other countries: a genome-wide association study. Lancet Neurol. 9, 986-994.

Smith, B.N., Ticozzi, N., Fallini, C., Gkazi, A.-S., Topp, S., Kenna, K.P., Scotter, E.L., Kost, J., Keagle, P., Miller, J.W., et al.; SLAGEN Consortium (2014). Exome-wide rare variant analysis identifies TUBA4A mutations associated with familial ALS. Neuron 84, 324-331.

Smith, E.F., Shaw, P.J., and De Vos, K.J. (2017). The role of mitochondria in amyotrophic lateral sclerosis. Neurosci. Lett. Published online June 30, 2017. https://doi.org/10.1016/j.neulet.2017.06.052.

Tanaka, Y., Kanai, Y., Okada, Y., Nonaka, S., Takeda, S., Harada, A., and Hirokawa, N. (1998). Targeted disruption of mouse conventional kinesin heavy chain, kif5B, results in abnormal perinuclear clustering of mitochondria. Cell 93, 1147-1158.

Taylor, J.P., Brown, R.H., Jr., and Cleveland, D.W. (2016). Decoding ALS: from genes to mechanism. Nature 539, 197-206.

Thiel, C., Kessler, K., Giessl, A., Dimmler, A., Shalev, S.A., von der Haar, S., Zenker, M., Zahnleiter, D., Stöss, H., Beinder, E., et al. (2011). NEK1 mutations cause short-rib polydactyly syndrome type majewski. Am. J. Hum. Genet. 88, 106-114.

Tompson, S.W.J., Ruiz-Perez, V.L., Blair, H.J., Barton, S., Navarro, V., Robson, J.L., Wright, M.J., and Goodship, J.A. (2007). Sequencing EVC and EVC2 identifies mutations in two-thirds of Ellis-van Creveld syndrome patients. Hum. Genet. 120, 663-670.

van Es, M.A., Veldink, J.H., Saris, C.G.J., Blauw, H.M., van Vught, P.W.J., Birve, A., Lemmens, R., Schelhaas, H.J., Groen, E.J.N., Huisman, M.H.B., et al. (2009). Genome-wide association study identifies 19p13.3 (UNC13A) and 9p21.2 as susceptibility loci for sporadic amyotrophic lateral sclerosis. Nat. Genet. 41, 1083-1087. van Es, M.A., Hardiman, O., Chio, A., Al-Chalabi, A., Pasterkamp, R.J., Veldink, J.H., and van den Berg, L.H. (2017). Amyotrophic lateral sclerosis. Lancet 390, 2084-2098.

van Rheenen, W., Shatunov, A., Dekker, A.M., McLaughlin, R.L., Diekstra, F.P., Pulit, S.L., van der Spek, R.A.A., Võsa, U., de Jong, S., Robinson, M.R., et al.; PARALS Registry; SLALOM Group; SLAP Registry; FALS Sequencing Consortium; SLAGEN Consortium; NNIPPS Study Group (2016). Genomewide association analyses identify new risk variants and the genetic architecture of amyotrophic lateral sclerosis. Nat. Genet. 48, 1043-1048.

Vance, C., Rogelj, B., Hortobágyi, T., De Vos, K.J., Nishimura, A.L., Sreedharan, J., Hu, X., Smith, B., Ruddy, D., Wright, P., et al. (2009). Mutations in FUS, an RNA processing protein, cause familial amyotrophic lateral sclerosis type 6 . Science 323, 1208-1211.

Wang, L., and Brown, A. (2010). A hereditary spastic paraplegia mutation in kinesin-1A/KIF5A disrupts neurofilament transport. Mol. Neurodegener. 5, 52-64. Wang, W.-Y., Pan, L., Su, S.C., Quinn, E.J., Sasaki, M., Jimenez, J.C., Mackenzie, I.R.A., Huang, E.J., and Tsai, L.-H. (2013). Interaction of FUS and HDAC1 regulates DNA damage response and repair in neurons. Nat. Neurosci. 16, 1383-1391.

Willer, C.J., Li, Y., and Abecasis, G.R. (2010). METAL: fast and efficient metaanalysis of genomewide association scans. Bioinformatics 26, 2190-2191.

Wu, C.-H., Fallini, C., Ticozzi, N., Keagle, P.J., Sapp, P.C., Piotrowska, K., Lowe, P., Koppers, M., McKenna-Yasek, D., Baron, D.M., et al. (2012). Mutations in the profilin 1 gene cause familial amyotrophic lateral sclerosis. Nature 488, 499-503.

Xia, C.-H., Roberts, E.A., Her, L.-S., Liu, X., Williams, D.S., Cleveland, D.W., and Goldstein, L.S.B. (2003). Abnormal neurofilament transport caused by targeted disruption of neuronal kinesin heavy chain KIF5A. J. Cell Biol. $161,55-66$. 


\section{STAR $\star$ METHODS}

\section{KEY RESOURCES TABLE}

\begin{tabular}{|c|c|c|}
\hline REAGENT or RESOURCE & SOURCE & IDENTIFIER \\
\hline \multicolumn{3}{|l|}{ Chemicals, Peptides, and Recombinant Proteins } \\
\hline TRIzol Reagent & Thermo Fisher Scientific & Cat\# 15596026 \\
\hline RNA-to-cDNA Kit & Applied Biosystems & Cat\# 4368814 \\
\hline RNase Inhibitor & Applied Biosystems & Cat\# 4374966 \\
\hline OneTaq Hot Start DNA Polymerase & New England BioLabs & Cat\# M0481S \\
\hline \multicolumn{3}{|l|}{ Critical Commercial Assays } \\
\hline HumanOmniExpress-24 DNA Analysis Kit & Illumina & Cat\# WG-312-3007 \\
\hline TruSeq Exome Enrichment Kit & Illumina & Cat\# FC-121-1096 \\
\hline TruSeq PE Cluster Kit & Illumina & Cat\# PE-401-3001 \\
\hline TruSeq SBS Kit & Illumina & Cat\# FC-401-3001 \\
\hline TrueSeq DNA PCR-free Kit & Illumina & Cat\# 20000902 \\
\hline HiSeq X Ten Reagent Kit & Illumina & Cat\# FC-501-2501 \\
\hline \multicolumn{3}{|l|}{ Oligonucleotides } \\
\hline F1 primer sequence: CAGTGGAGCCACATCTTCTG & Operon Technologies & N/A \\
\hline R1 primer sequence: TCTCTTGGTGGAGAGGGAAA & Operon Technologies & $\mathrm{N} / \mathrm{A}$ \\
\hline F2 primer sequence: CCAACATGGACAATGGAGTGA & Operon Technologies & N/A \\
\hline \multicolumn{3}{|l|}{ Software and Algorithms } \\
\hline ASSEDA & Tompson et al., 2007 & $\begin{array}{l}\text { http://www.cytognomix.com/?post_ } \\
\text { type=duka\&p=2670 }\end{array}$ \\
\hline BWA & Wellcome Trust Sanger Institute & http://bio-bwa.sourceforge.net \\
\hline GenABEL & The GenABEL Project & http://www.genabel.org \\
\hline GATK & Broad Institute & https://software.broadinstitute.org/gatk/ \\
\hline id_geno_checksum & Broad Institute & $\begin{array}{l}\text { https://personal.broadinstitute.org/sripke/ } \\
\text { share_links/checksums_download/ }\end{array}$ \\
\hline KING & Manichaikul et al., 2010 & http://people.virginia.edu/ wc9c/KING/ \\
\hline LASER & University of Michigan & http://csg.sph.umich.edu/chaolong/LASER/ \\
\hline Mach2dat & Marchini and Howie, 2010 & $\begin{array}{l}\text { https://genome.sph.umich.edu/wiki/ } \\
\text { Mach2dat:_Association_with_MACH_output }\end{array}$ \\
\hline METAL & Willer et al., 2010 & $\begin{array}{l}\text { http://csg.sph.umich.edu/abecasis/metal/ } \\
\text { index.html }\end{array}$ \\
\hline Minimac3 & Das et al., 2016 & https://genome.sph.umich.edu/wiki/Minimac3 \\
\hline PLINK & Chang et al., 2015 & http://zzz.bwh.harvard.edu/plink/ \\
\hline$R$ & R Core Team & https://www.r-project.org \\
\hline SHAPEIT & Delaneau et al., 2013 & $\begin{array}{l}\text { https://mathgen.stats.ox.ac.uk/genetics_ } \\
\text { software/shapeit/shapeit.html }\end{array}$ \\
\hline dbNSFP & Liu et al., 2013 & $\begin{array}{l}\text { http://varianttools.sourceforge.net/ } \\
\text { Annotation/DbNSFP }\end{array}$ \\
\hline \multicolumn{3}{|l|}{ Other } \\
\hline Genotyped data & Current paper & $\begin{array}{l}\text { https://www.ncbi.nlm.nih.gov/projects/ } \\
\text { gap/cgi-bin/study.cgi?study_id= } \\
\text { phs000101.v4.p1 }\end{array}$ \\
\hline
\end{tabular}




\section{CONTACT FOR REAGENT AND RESOURCE SHARING}

Further information and requests for resources and reagents should be directed to and will be fulfilled by the Lead Contact, John Landers (john.landers@umassmed.edu).

\section{EXPERIMENTAL MODEL AND SUBJECT DETAILS}

\section{Study cohorts \\ GWAS cohort I}

We undertook a GWAS of patients diagnosed with ALS (case cohort) and neurologically normal control individuals (control cohort). DNA was extracted from either whole blood or frozen brain tissue samples using standard procedures. All 12,663 patients included in the case cohort had been diagnosed with ALS according to the El Escorial criteria (Brooks, 1994) by a neurologist specializing in ALS, had onset of symptoms after age 18 years, and were of non-Hispanic white race/ethnicity. Both patients with familial ALS and patients with sporadic ALS were included in the analysis.

For the control cohort, we used genotype data obtained from (a) the database of Genotypes and Phenotypes (dbGaP) web repository ( $n=44,017$ US samples); (b) the HYPERGENES Project ( $n=887$ Italian samples) (Salvi et al., 2012); and (c) the Wellcome Trust Case Control Consortium ( $n=5,663$ British samples). An additional 2,112 US and Italian control samples were genotyped in the Laboratory of Neurogenetics, National Institute on Aging. The control cohort was matched to the case cohort for race and ethnicity, but not for age or sex. A detailed description of the cohorts is available in Tables S1 and S2.

Written consent was obtained from all individuals enrolled in this study, and the study was approved by the institutional review board approval of the National Institute on Aging (protocol number 03-AG-N329).

\section{GWAS cohort II}

Summary statistics from a recently published GWAS based on logistic regression analysis involving 12,577 cases and 23,475 controls were downloaded from the Project MinE Variant Browser. Additional details of the cohorts used in this study are available in van Rheenen et al. (2016).

\section{FALS discovery cohort}

A total of 1,463 FALS patients were included in the initial cohort (pre-QC). Patients were recruited at specialist clinics in Australia ( $n=92)$, Belgium $(n=13)$, Canada $(n=34)$, Germany $(n=228)$, Ireland $(n=18)$, Israel $(n=26)$, Italy $(n=230)$, Netherlands $(n=50)$, Spain $(n=60)$, Turkey $(n=72)$, UK $(n=223)$, and USA $(n=417)$. All samples were exome sequenced except those from the Netherlands which were whole genome sequenced. Familial history was considered positive for ALS if the proband had at least one affected relative within three degrees of relatedness.

\section{Control discovery cohort}

Read level sequencing data were obtained from dbGAP and the European Genome-Phenome Archive (EGA) and are listed in Table S5.

\section{ALS WXS/WGS replication cohort}

Replication analyses included sequencing data for a further 9,046 ALS cases and 1,955 non-ALS controls that were not also represented in the FALS discovery set. These samples included 2,742 cases subjected to WXS by the ALS Sequencing Consortium, as described previously (Cirulli et al., 2015); 719 cases subjected to WXS by the Laboratory of Neurogenetics, National Institute on Aging; 307 cases and 296 controls subjected to WGS by the Laboratory of Neurogenetics, National Institute on Aging; 155 cases subjected to WGS by the CReATe Consortium; 1,017 cases subjected to WGS by the NYGC ALS Consortium, Genomic Translation for ALS Care (GTAC) Consortium and Answer ALS Foundation; and 4,100 cases and 1,659 controls subjected to WGS by the Project MinE Sequencing Consortium.

All samples included in the case cohort had been diagnosed with ALS according to the El Escorial criteria (Brooks, 1994) by a neurologist specializing in ALS. We received approval for this study from the institutional review boards of the participating centers, and written informed consent was obtained from all patients (consent for research).

\section{METHOD DETAILS}

\section{Data generation and pre-processing}

\section{Generation of SNP array callset}

The case cohort ( $n=12,663$ samples) and part of the control cohort $(n=2,112)$ were genotyped in the Laboratory of Neurogenetics, National Institute on Aging, using HumanOmniExpress BeadChips (version 1.0, Illumina, San Diego, CA) according to the manufacturer's protocol. These SNP genotyping arrays assay 716,503 SNPs across the genome. Individual-level genotypes for these samples are available on the dbGaP web portal (accession number phs000101.v4.p1). The remainder of the control cohort had been previously genotyped on HumanOmni BeadChips (Illumina) as part of other GWAS efforts (Table S2). Analyses were confined to the 595,692 autosomal SNPs that were common across the SNP genotyping arrays. 
Generation of FALS case-control callset for exome-wide RVB discovery analysis

Exome sequencing of cases was performed as previously described (Kenna et al., 2016). Control exome sequences were generated as described under the relevant dbGAP and EGA project accessions. Sequence reads were aligned to human reference GRCh37 using BWA (Burrows-Wheeler Aligner) and processed according to recommended Genome Analysis Toolkit's (GATK) best practices (https://software.broadinstitute.org/gatk/best-practices/). Joint variant detection and genotyping of all samples were performed using the GATK HaplotypeCaller. Variant quality control was performed using the GATK variant quality score recalibration method with default filters. A minimum variant quality by depth (QD) score of 2 was also imposed and all genotypes associated with genotype quality $(\mathrm{GQ})<20$ were reset to missing. Variants were also excluded in the event of case or control call rates $<70 \%$ (post genotype QC). Identified variants can be viewed through our web based ALS Variant Server (see link below).

Generation of ALS case-control callset for KIF5A replication analysis

Data for the KIF5A locus was extracted from all independently generated sequencing datasets and remapped to GRCh37. Variant calling was performed using the GATK haplotype caller as described above. In addition to the KIF5A locus, data was also extracted for a panel of 240,715 common variant sites and used to perform a single unified sample QC as described below.

\section{Functional annotation of variants identified by WXS/WGS}

Variant calls were assigned predicted functional consequences using snpEFF (Single Nucleotide Polymorphism Effect) (Cingolani et al., 2012), dbNSFP (A Database of Human Non-synonymous SNVs and Their Functional Predictions and Annotations) (Liu et al., 2013) and dbscSNV (database of splice site consequences of Single Nucleotide Variants) (Jian et al., 2014), which is incorporated into dbNSFP. Variants were classified as "loss of function" (LOF) where the sequence change was predicted to encode a premature stop codon, a frameshift causing insertion-deletion or a splice site disrupting SNV. Variants were classified as potentially splice altering if assigned an "ada" or "rf" score $>0.7$ by dbscSNV. Splice variants of potential interest were further assessed for putative effects on exon skipping using a secondary algorithm - automated splice site and exon definition server (ASSEDA) (Tompson et al., 2007).

\section{RT-PCR Analysis}

Total RNA was prepared from lymphoblast lines using Trizol reagent. Reverse transcription using Applied Biosystems RNA to cDNA kit (\# 4368814) was performed with $0.5 \mu \mathrm{g}$ with RNase inhibitor in a $20 \mu$ reaction according to the manufacturer's protocol. PCR was carried out using New England Biolabs One Taq Hot Start DNA Polymerase (\# M0481S), $2 \mu \mathrm{l}$ RT reaction (representing 50 ng input $\mathrm{RNA})$ and forward and reverse primer $\left(0.15 \mu \mathrm{M}\right.$ each) in a $20 \mu \mathrm{l}$ reaction volume. Amplification conditions were as follows: $94^{\circ} \mathrm{C}$ for $30 \mathrm{~s},\left\{94^{\circ} \mathrm{C}\right.$ for $20 \mathrm{~s}, 58^{\circ} \mathrm{C}$ for $20 \mathrm{~s}, 68^{\circ} \mathrm{C}$ for 1 minute $\} \times 35$ cycles, followed by an extension stage of $68^{\circ} \mathrm{C}$ for 5 minutes and a $4^{\circ} \mathrm{C} \mathrm{hold}$. Amplification of both normal and mutant splice forms used primers F1 (CAGTGGAGCCACATCTTCTG) and R1 (TCTCTTGGTGGA GAGGGAAA). Primers used for the specific amplification of the mutant splice form were F2 (CCAACATGGACAATGGAGTGA), which spans exons 26 and 28 , and $\mathrm{R} 1$.

\section{QUANTIFICATION AND STATISTICAL ANALYSIS}

\section{Statistical analyses}

\section{Analysis of SNP array genotypes}

Standard quality-control procedures were applied to our genotype data using PLINK software package (version 1.9) (Chang et al., 2015), and a summary of the workflow is shown in Figure S1. We excluded samples that demonstrated: call rates of less than 97.5\%; non-European ancestry; abnormal $F$ inbreeding coefficient; mismatch between phenotypic and genotypic gender; or, cryptic relatedness defined as identity-by-descent proportion of inheritance (pi_hat from PLINK) greater than 0.125. Samples in common between our study and van Rheenen's study were identified using the checksum program id_geno_checksum and were removed from our analyses. We excluded palindromic SNPs, as well as SNPs with: call rates less than 95\% in the US and Italian cohorts or less than $99 \%$ in the UK, French and Belgium cohorts; minor allele frequency less than 0.05 in the control cohorts; Hardy-Weinberg equilibrium $P$ less than $10^{-7}$ in the US and Italian control cohorts and less than $10^{-5}$ in the UK, French and Belgium cohorts; missingness by case-control status $P$ less than $10^{-5}$; or SNPs associated between the UK and French control cohorts with $P$ less than $5.0 \times 10^{-8}$. After quality control, 8,229 case and 36,329 control samples were included in the analysis, and 436,746 SNPs were available for imputation in the USA and Italy cohorts, and 420,131 SNPs were available in the UK, French and Belgium cohorts.

Estimation of the haplotypes was performed with SHAPEIT (version 2.r790) (Delaneau et al., 2013). Imputation was performed for individual batches based on ethnicity using the 1000 Genomes Project dataset (phase 3, version 5a, release 2013-05-02, http://www. internationalgenome.org/) as reference and using Minimac3 software (version 1.0.11) (Das et al., 2016) with default settings. After imputation, principal components were calculated using PLINK software after removing known hypervariable regions and the $1 \mathrm{MB}$ surrounding the C9orf72 region. After analysis of the Scree plots, 2 to 4 principal components were retained per cohort as covariates in the association analyses to compensate for any residual population stratification.

Logistic regression was performed per batch using mach2dat software (version 1.0.24) (Marchini and Howie, 2010) incorporating 2 to 4 principal components, age and gender as covariates, with dosage of imputed SNPs selected based on a Minimac3 $R^{2}$ value of imputation accuracy greater than 0.3. SNPs with an absolute beta coefficient value above 5 or with a minor allele frequency less 
than 0.01 were excluded from meta-analysis. Meta-analysis was then performed combining the association results of the 13 batches of our individual-level studies with van Rheenen's study summary statistics using METAL software (version 2011-03-25) (Willer et al., 2010) under an inverse-weighted, fixed effect model. A threshold $P$ of $5.0 \times 10^{-8}$ was set for genome-wide significance after Bonferroni correction for multiple testing in the GWAS (Pe'er et al., 2008).

The programming code used to analyze these data is freely available on GitHub (https://github.com/AudeDN/ALS_GWAS_ 1000G_mach2dat_2017), and GWAS summary statistics results for all tested SNPs are available from https://www.ncbi.nlm.nih. gov/projects/gap/cgi-bin/study.cgi?study_id=phs000101.v4.p1.

\section{Analysis of WXS/WGS genotypes}

For both the discovery and replication phases, samples were excluded from the study in the event of failing to meet standard genotype call rate, heterozygosity, duplication, relatedness or population stratification filters as summarized in Table S6. Each of these filters was performed using a set of autosomal markers meeting all of the following criteria: call rate $>0.95$, minor allele frequency $(\mathrm{MAF})>0.01, \mathrm{p}>0.001$ for deviation from Hardy-Weinberg equilibrium, linkage disequilibrium pruning $\left(\mathrm{R}^{2}<0.5\right.$, window size $=$ 50 , step $=5$ ). Filtering of autosomal markers, sample call rate assessments and sample heterozygosity assessments were performed using PLINK software. Study duplicates and sample relatedness within the WXS/WGS cohorts was identified using KING software (Manichaikul et al., 2010). Study duplicates between WXS/WGS cohorts and GWAS datasets were identified using the checksum program id_geno_checksum. LASER was used to generate PCA coordinates for samples from the Human Genome Diversity Panel (HGDP). Samples from the FALS discovery cohort were then mapped to this reference co-ordinate space. The discovery cohort was restricted to cases and controls occurring within 3 standard deviations of the mean for European HGDP samples along principal components 1-4.

RVB analyses were performed by penalized logistic regression of case-control status with respect to number of minor alleles observed per sample per gene with and MAF $<0.001$. Analyses were only performed where the dataset contained more than 3 variant allele occurrences. Replication analyses of rs 113247976 were performed using the same logistic regression protocol as used for RVB analyses. All analyses were conditioned on the first 4 eigenvectors generated by principal components analysis of common variant profiles. Genomic inflation factors were calculated using genome-wide association analysis for quantitative, binary and time-tillevent traits using GenABEL software. Candidate associations were tested for signs of call-rate or subcohort biases as outlined in Figures S6 and S7. Meta-analysis of rs113247976 association results between sequencing and GWAS was performed using METAL. Unless otherwise indicated, all statistical analyses were performed using R (version 3.2.0).

\section{Control-control analyses}

To identify genes potentially subject to confounding biases in FALS RVB analyses and to assess the potential impact of batch effects with non-ALS-related data, population or phenotypic stratifiers, the control sample cohort was divided into 28 pseudo case-control groups based on the sequencing center or associated dbGaP / EGA project (Table S5). Genes shown in gray achieve for possible confounder association. Loci achieving a minimum $\mathrm{p}<1 \times 10^{-3}$ were deemed as displaying possible association with non-ALS related batch effects.

\section{DATA AND SOFTWARE AVAILABILITY}

\section{Datasets}

The programming code used to analyze the GWAS data including the imputation with SHAPEIT and Minimac3, individual-based association analysis using Mach2dat and a meta-analysis using METAL is freely available on GitHub: https://github.com/AudeDN/ ALS_GWAS_1000G_mach2dat_2017). GWAS summary statistics results for all tested SNPs and identified SNVs from our 1,138 FALS cohort used for the RVB analysis can be viewed through our web based ALS Variant Server (http://als.umassmed.edu). For each variant, information on over 50 annotation fields and the results can be downloaded directly into Excel. The accession number for the genotyped data reported in this paper is dbGaP: phs000101.v4 (https://www.ncbi.nlm.nih.gov/projects/gap/cgi-bin/study. cgi?study_id=phs000101.v4.p1).

\section{Data Resources and Databases}

1000 Genomes Project dataset: http://www.internationalgenome.org

Database of Genotypes and Phenotypes (dbGaP): https://www.ncbi.nlm.nih.gov/gap

dbNSFP: https://sites.google.com/site/jpopgen/dbNSFP

dbscSNV: incorporated into dbNSFP (see previous link).

European Genome-phenome Archive (EGA): https://ega-archive.org

HapMap project: http://www.sanger.ac.uk/resources/downloads/human/hapmap3.html.

Human Genome Diversity Panel (HGDP): http://www.hagsc.org/hgdp/

HYPERGENES Project: https://cordis.europa.eu/project/rcn/86758_en.html

Project MinE Variant Browser: http://databrowser.projectmine.com

snpEFF: http://snpeff.sourceforge.net/SnpEff.html

Wellcome Trust Case Control Consortium: https://www.wtccc.org.uk/ 


\section{Software}

ASSEDA: http://www.cytognomix.com/?post_type=duka\&p=2670

BWA: http://bio-bwa.sourceforge.net

GenABEL: http://www.genabel.org

GATK: https://software.broadinstitute.org/gatk/

id_geno_checksum: https://personal.broadinstitute.org/sripke/share_links/checksums_download/

KING: http://people.virginia.edu/ wc9c/KING/

LASER: http://csg.sph.umich.edu/chaolong/LASER/

Mach2dat: https://genome.sph.umich.edu/wiki/Mach2dat:_Association_with_MACH_output

METAL: http://csg.sph.umich.edu/abecasis/metal/index.html

Minimac3: https://genome.sph.umich.edu/wiki/Minimac3

PLINK: http://zzz.bwh.harvard.edu/plink/

R: https://www.r-project.org

SHAPEIT: https://mathgen.stats.ox.ac.uk/genetics_software/shapeit/shapeit.html 
Neuron, Volume 97

Supplemental Information

\section{Genome-wide Analyses Identify}

\section{KIF5A as a Novel ALS Gene}

Aude Nicolas, Kevin P. Kenna, Alan E. Renton, Nicola Ticozzi, Faraz Faghri, Ruth Chia, Janice A. Dominov, Brendan J. Kenna, Mike A. Nalls, Pamela Keagle, Alberto M. Rivera, Wouter van Rheenen, Natalie A. Murphy, Joke J.F.A. van Vugt, Joshua T. Geiger, Rick A. Van der Spek, Hannah A. Pliner, Shankaracharya, Bradley N. Smith, Giuseppe Marangi, Simon D. Topp, Yevgeniya Abramzon, Athina Soragia Gkazi, John D. Eicher, Aoife Kenna, ITALSGEN Consortium, Gabriele Mora, Andrea Calvo, Letizia Mazzini, Nilo Riva, Jessica Mandrioli, Claudia Caponnetto, Stefania Battistini, Paolo Volanti, Vincenzo La Bella, Francesca L. Conforti, Giuseppe Borghero, Sonia Messina, Isabella L. Simone, Francesca Trojsi, Fabrizio Salvi, Francesco 0. Logullo, Sandra D'Alfonso, Lucia Corrado, Margherita Capasso, Luigi Ferrucci, Genomic Translation for ALS Care (GTAC) Consortium, Cristiane de Araujo Martins Moreno, Sitharthan Kamalakaran, David B. Goldstein, ALS Sequencing Consortium, Aaron D. Gitler, Tim Harris, Richard M. Myers, NYGC ALS Consortium, Hemali Phatnani, Rajeeva Lochan Musunuri, Uday Shankar Evani, Avinash Abhyankar, Michael C. Zody, Answer ALS Foundation, Julia Kaye, Steven Finkbeiner, Stacia K. Wyman, Alex LeNail, Leandro Lima, Ernest Fraenkel, Clive N. Svendsen, Leslie M. Thompson, Jennifer E. Van Eyk, James D. Berry, Timothy M. Miller, Stephen J. Kolb, Merit Cudkowicz, Emily Baxi, Clinical Research in ALS and Related Disorders for Therapeutic Development (CReATe) Consortium, Michael Benatar, J. Paul Taylor, Evadnie Rampersaud, Gang Wu, Joanne Wuu, SLAGEN Consortium, Giuseppe Lauria, Federico Verde, Isabella Fogh, Cinzia Tiloca, Giacomo P. Comi, Gianni Sorarù, Cristina Cereda, French ALS Consortium, Philippe Corcia, Hannu Laaksovirta, Liisa Myllykangas, Lilja Jansson, Miko Valori, John Ealing, Hisham Hamdalla, Sara Rollinson, Stuart Pickering-Brown, Richard W. Orrell, Katie C. Sidle, Andrea Malaspina, John Hardy, Andrew B. Singleton, Janel O. Johnson, Sampath Arepalli, Peter C. Sapp, Diane McKenna-Yasek, Meraida Polak, Seneshaw Asress, Safa AlSarraj, Andrew King, Claire Troakes, Caroline Vance, Jacqueline de Belleroche, Frank Baas, Anneloor L.M.A. ten Asbroek, José Luis Muñoz-Blanco, Dena G. Hernandez, Jinhui Ding, J. Raphael Gibbs, Sonja W. Scholz, Mary Kay Floeter, Roy H. Campbell, Francesco Landi, Robert Bowser, Stefan M. Pulst, John M. Ravits, Daniel J.L. MacGowan, Janine Kirby, Erik P. Pioro, Roger Pamphlett, James Broach, Glenn Gerhard, Travis L. Dunckley, Christopher B. Brady, Neil W. Kowall, Juan C. Troncoso, Isabelle Le Ber, Kevin Mouzat, Serge Lumbroso, Terry D. Heiman-Patterson, Freya Kamel, Ludo Van Den Bosch, Robert H. Baloh, Tim M. Strom, Thomas Meitinger, Aleksey Shatunov, Kristel R. Van Eijk, Mamede de Carvalho, Maarten Kooyman, Bas Middelkoop, Matthieu Moisse, Russell L. McLaughlin, Michael A. Van Es, Markus Weber, Kevin B. Boylan, Marka Van Blitterswijk, Rosa Rademakers, Karen E. Morrison, A. Nazli Basak, Jesús S. Mora, Vivian E. Drory, Pamela J. Shaw, Martin R. Turner, Kevin Talbot, Orla Hardiman, Kelly L. Williams, Jennifer A. Fifita, Garth A. Nicholson, Ian P. Blair, Guy A. 


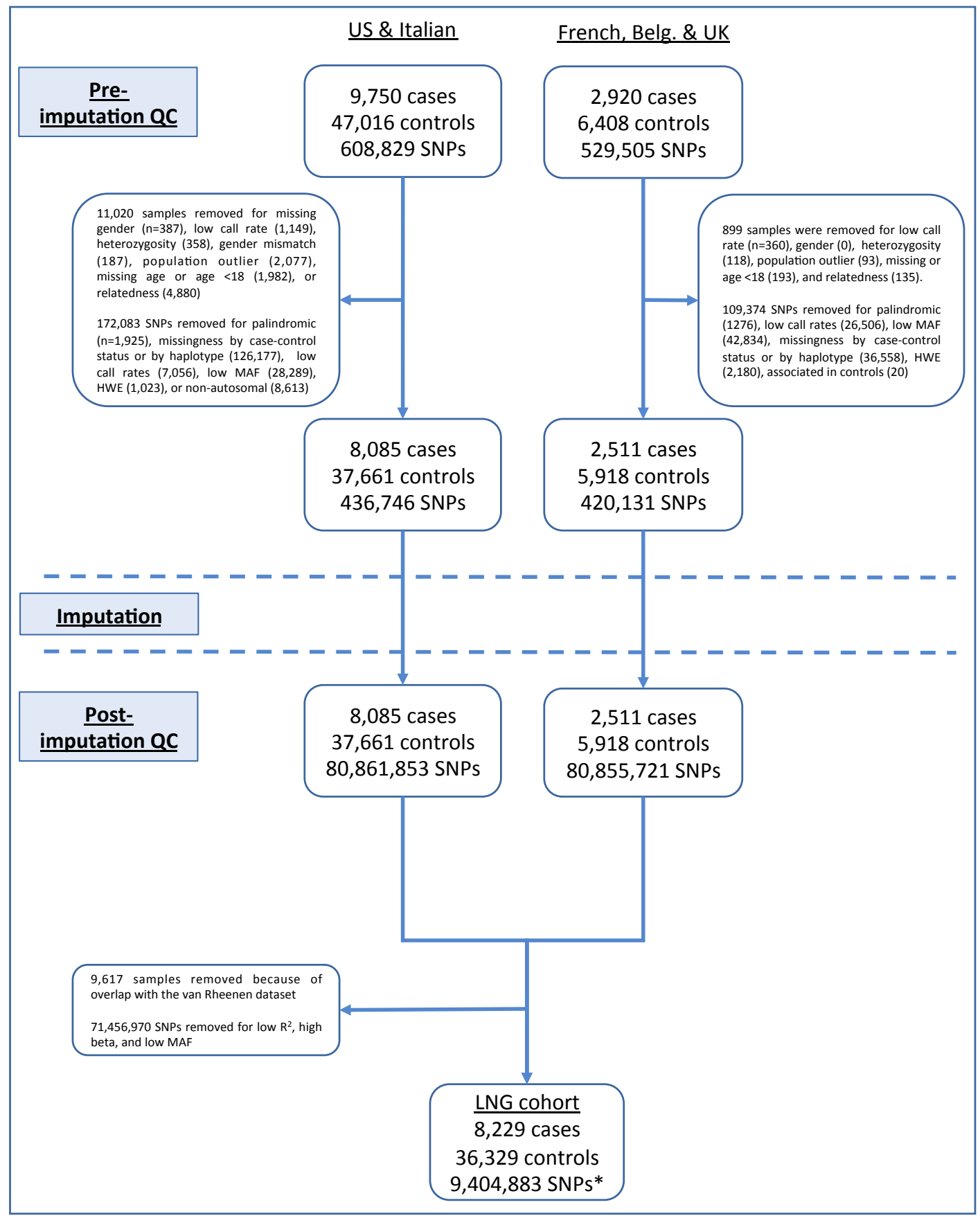

Figure S1. Related to Figure 1; Workflow showing the quality control procedures applied to the present study. *increased to 10,031,630 when merged with the Van Rheenen et al dataset; Belg., Belgium; SNP, single nucleotide polymorphism; MAF, minor allele frequency, HWE, HardyWeinberg equilibrium; $\mathrm{R}^{2}$, R-square value representing imputation precision; LNG, Laboratory of Neurogenetics. 


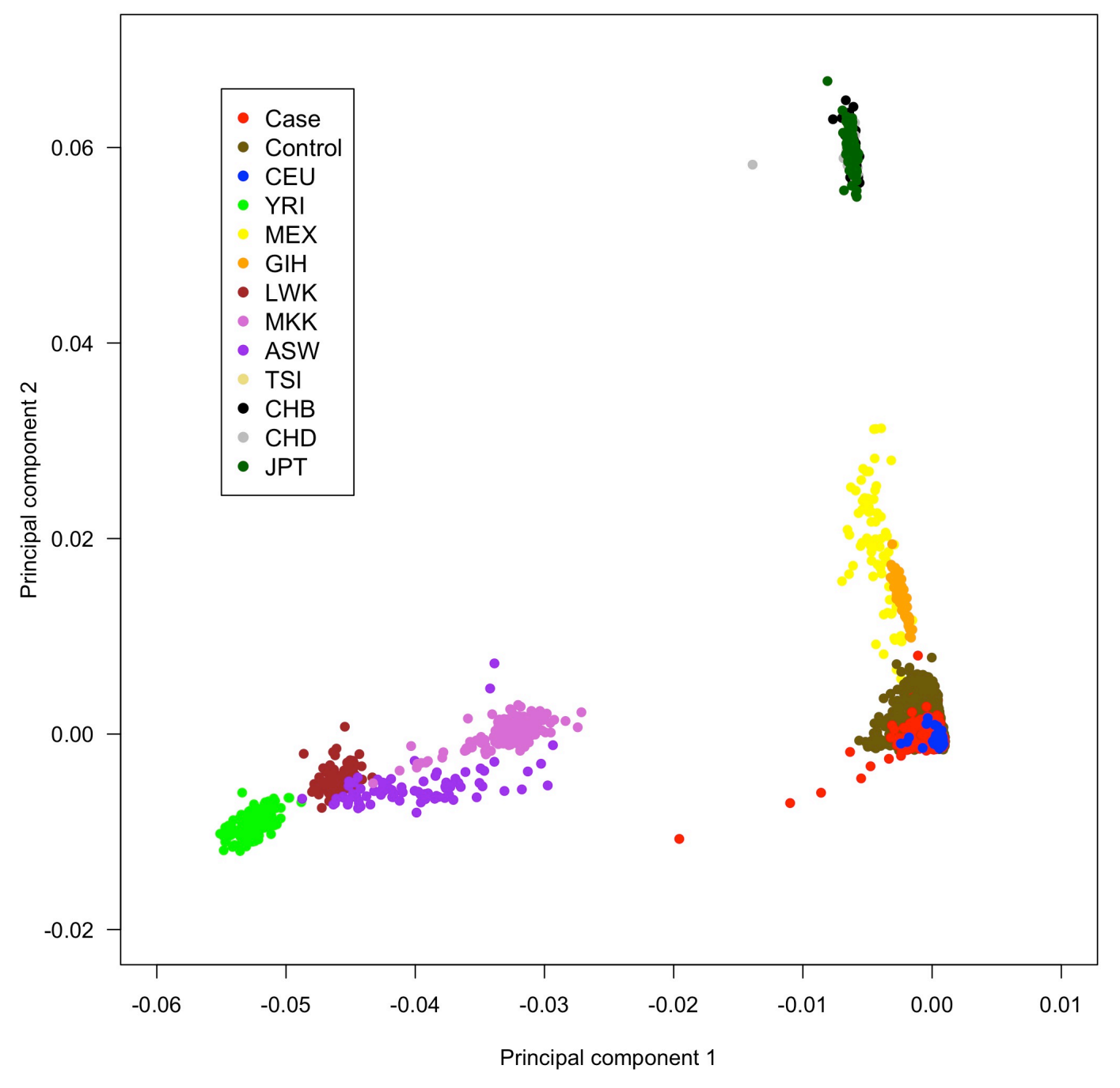

Figure S2. Related to Figure 1; Multi-dimensional scaling plot of the 44,558 genotyped samples included in analysis compared to the HapMap populations. 


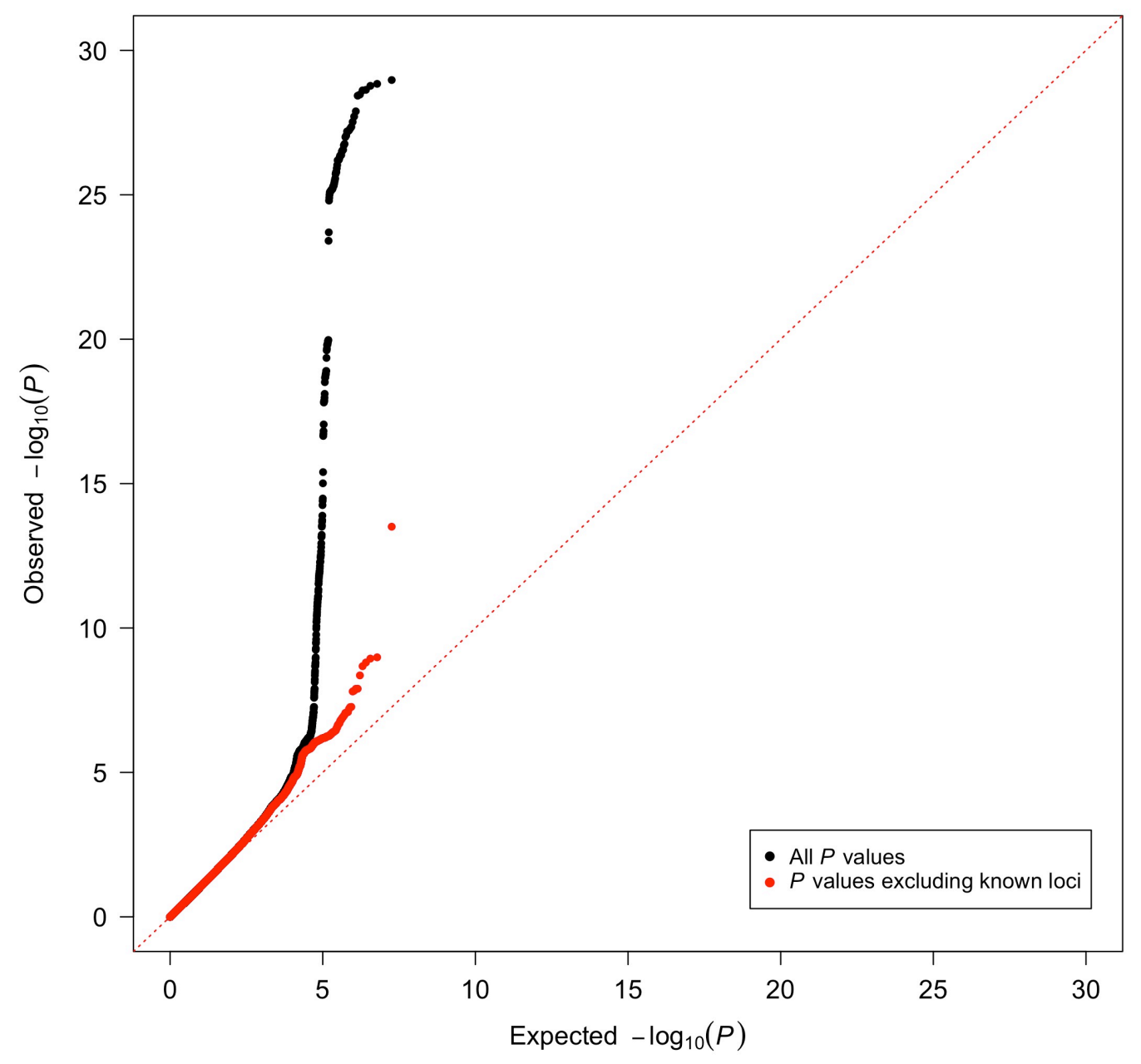

Figure S3. Related to Figure 1; Quartile-Quartile plot of $\boldsymbol{P}$-values from the meta-analysis based on logistic regression analysis. The black curve represents all SNPs, and the red curve represent SNPs after excluding variants within +/- 500 kilobases of the C9orf72 and the UNC13A loci. Raw genome inflation factor $(\lambda)$ was 1.042 and adjusted $\lambda$ scaled to 1,000 cases and 1,000 controls was 1.001 based on the entire SNP dataset. 

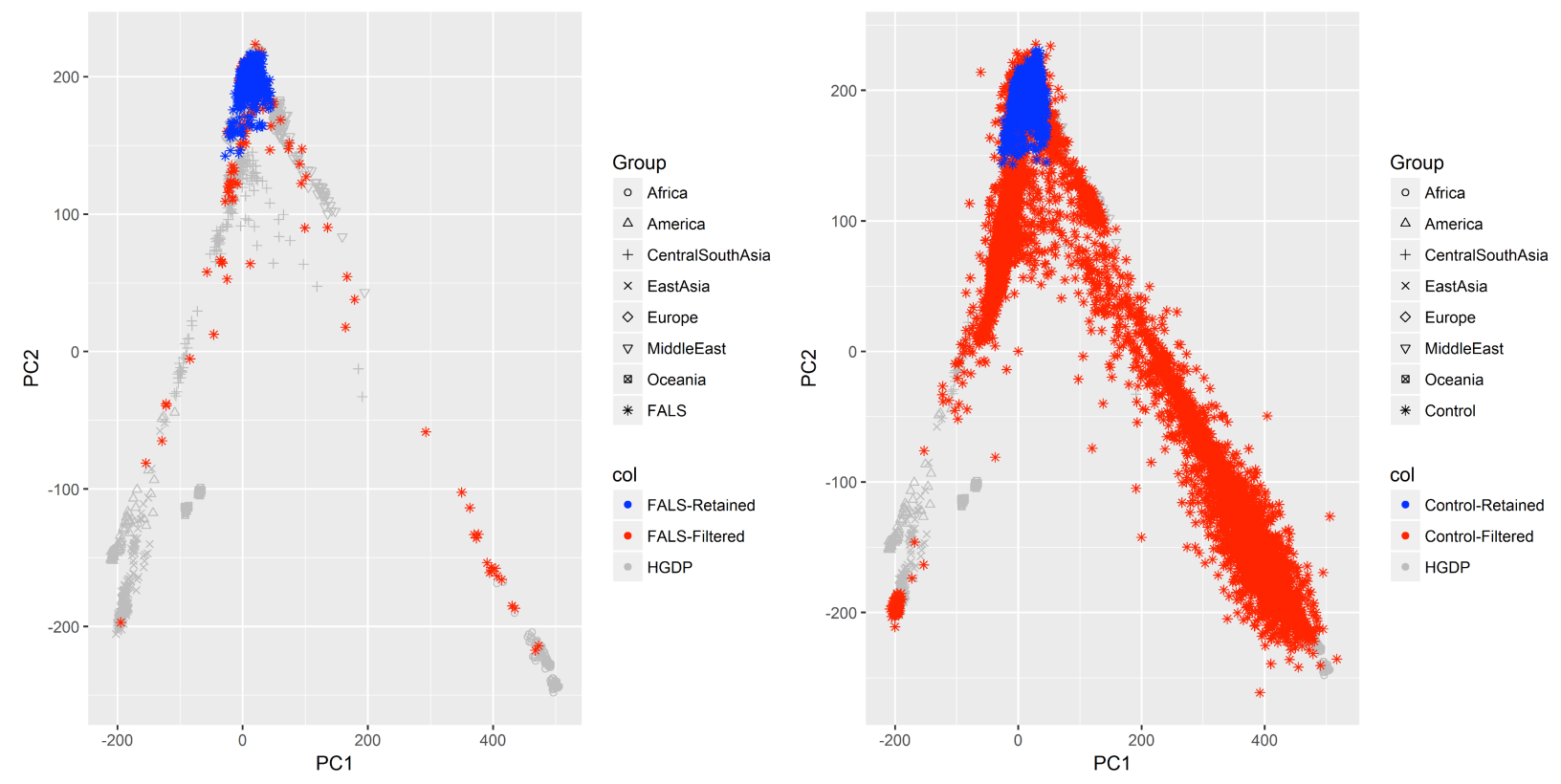

Figure S4. Related to Figure 3; Principal components analysis of samples included in the RVB analysis compared to the Human Diversity Panel. Ancestry filtering of the FALS discovery cohort was performed as follows: LASER was used to generate PCA coordinates for samples from the Human genome diversity panel (HGDP). Samples from the FALS discovery cohort were then mapped to this reference co-ordinate space. The discovery cohort was restricted to cases and controls occurring within 3 standard deviations of the mean for European HGDP samples along principal components 1-4. 


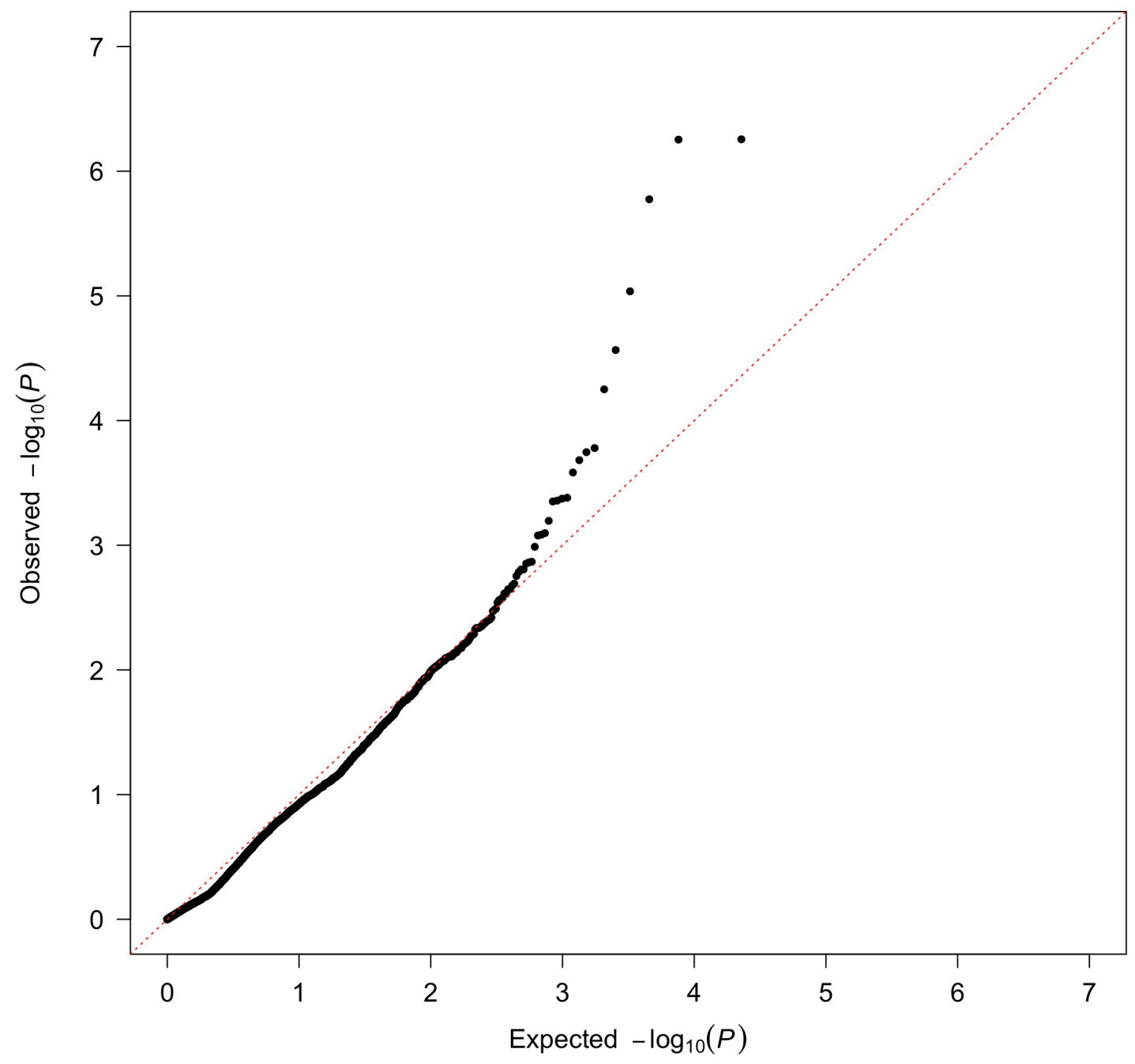

Figure S5. Related to Figure 3; Quartile-Quartile plot of $\mathbf{P}$ values from the gene-based rare variant burden analysis of exome data. The genomic inflation factor $(\lambda=0.93)$ was calculated based on the entire gene dataset. 


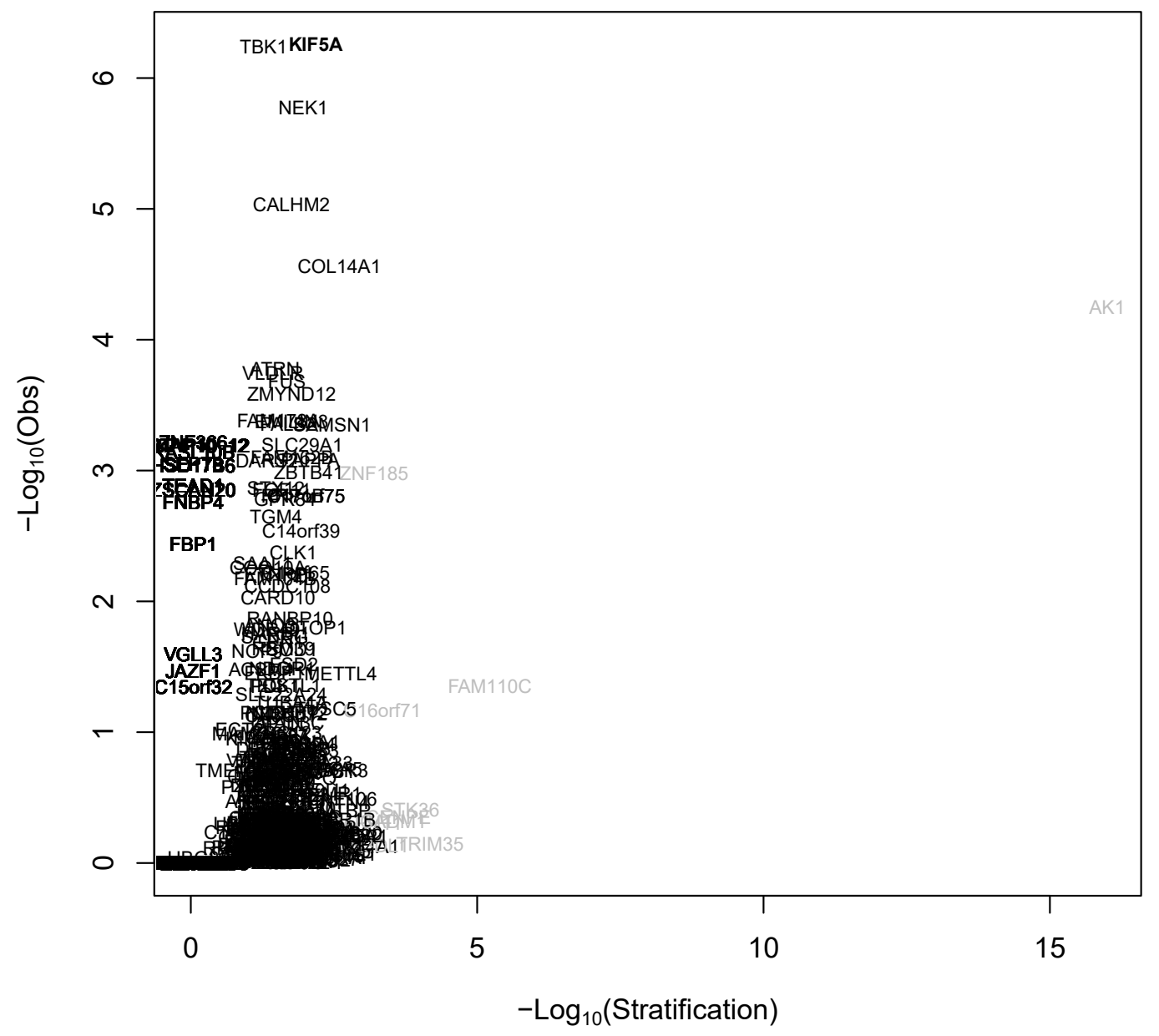

Figure S6. Related to Figure 3; Control-control analyses. $P$ values from RVB analysis of FALS cases versus controls (y-axis) are plotted against minimum $P$ values from RVB analyses of candidate batch effects (x-axis). To assess the potential impact of batch effects, the sample cohort was divided into 28 pseudo case-control groups based on the sequencing center or associated dbGaP project. Loci showing possible association with non-ALS related batch effects are coloured light grey. No evidence of confounder bias was observed for KIF5A or previously reported ALS genes. 


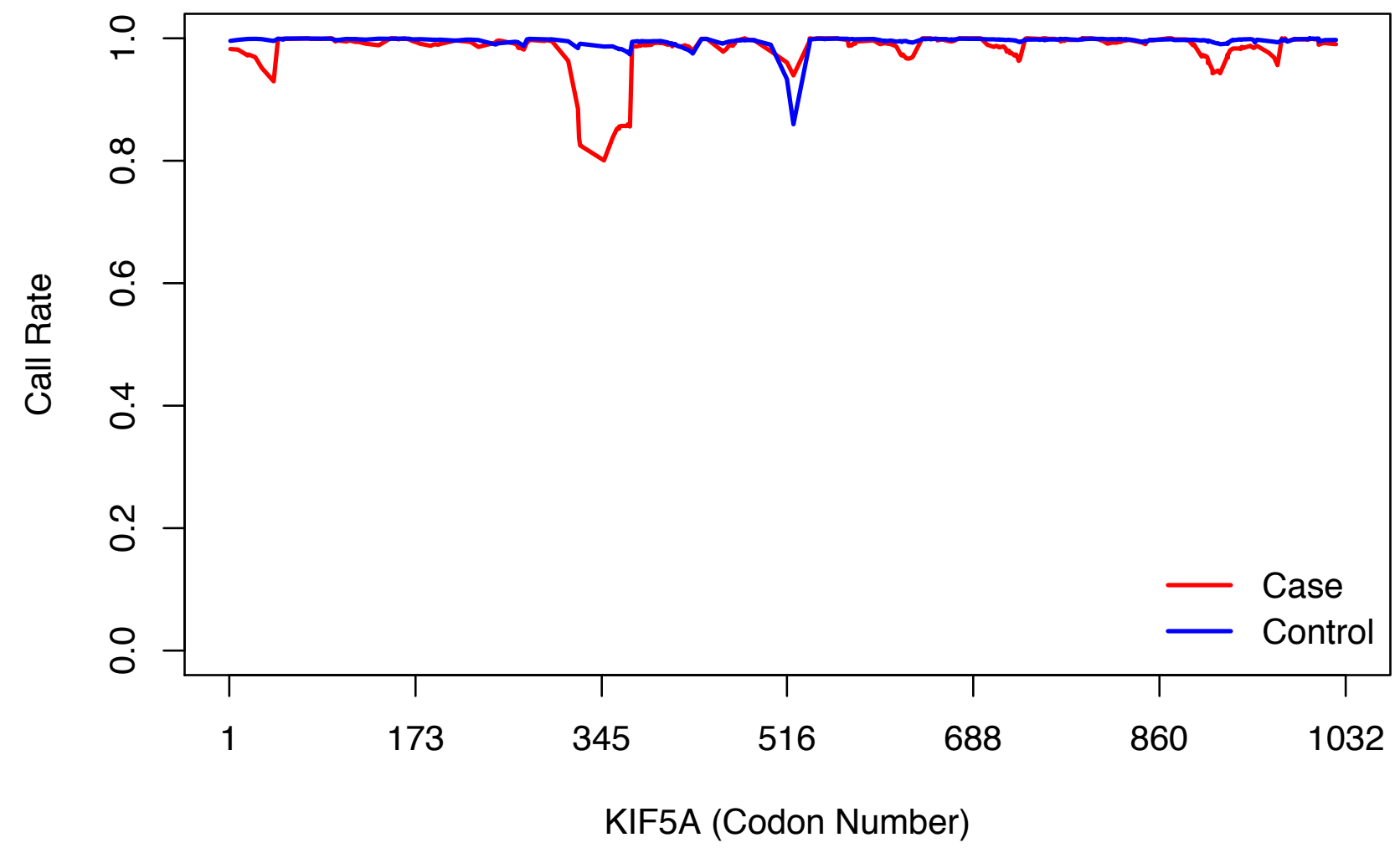

Figure S7. Related to Fgure 3; Plot of variant call rates across the KIF5A protein-coding region in FALS versus controls analyzed by RVB testing. 


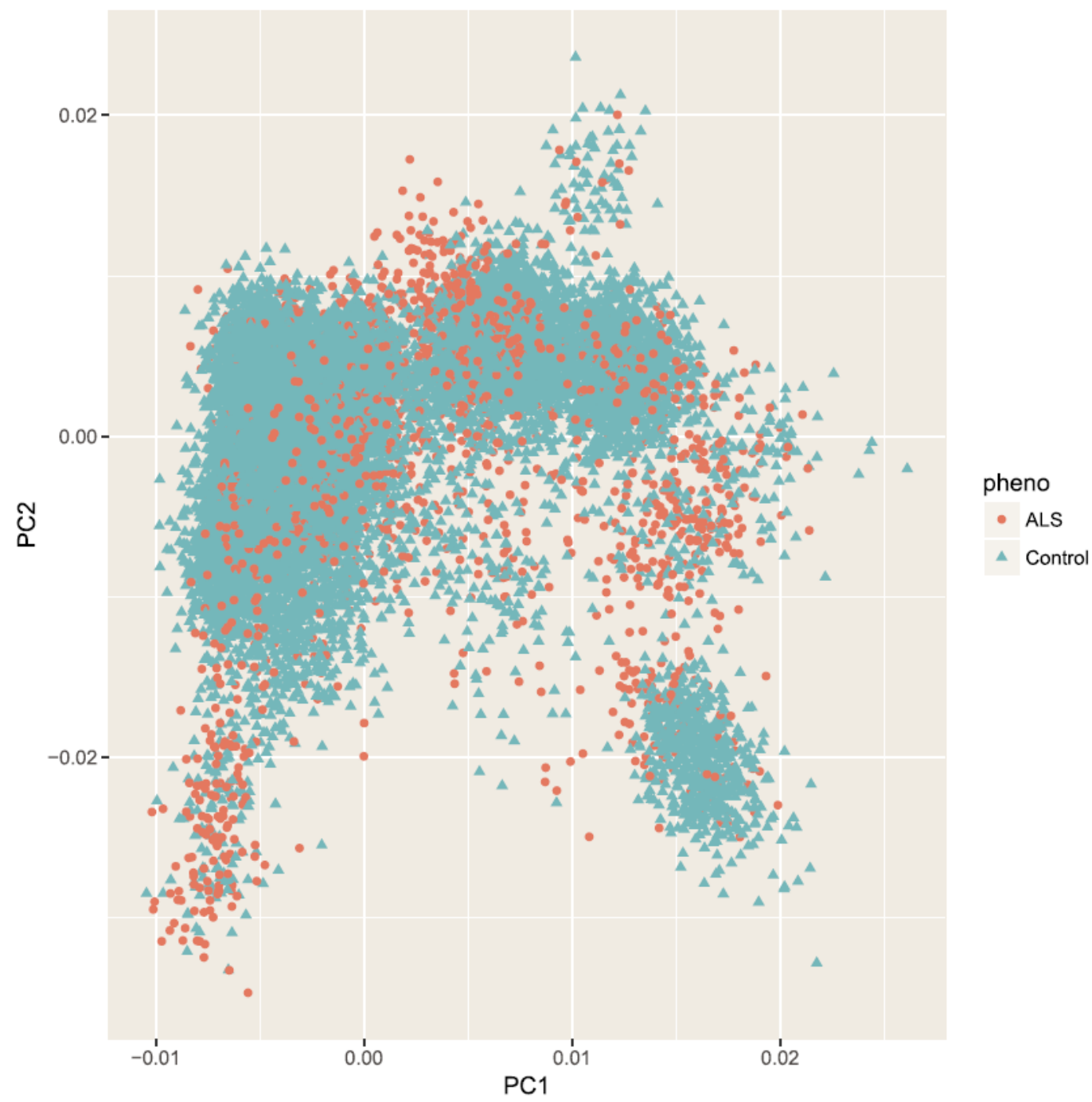

Figure S8. Related to Figure 2; Principal components analysis of samples included in KIF5A replication cohort. 
Table S1. Related to Figure 1; Demographics and baseline characteristics of patients diagnosed with ALS and control individuals included in the GWAS analysis.

\begin{tabular}{|c|c|c|c|c|c|c|c|c|c|c|}
\hline & \multicolumn{2}{|c|}{$\mathbf{U S}$} & \multicolumn{2}{|c|}{ Italian } & \multicolumn{2}{|c|}{$\mathbf{U K}$} & \multicolumn{2}{|c|}{$\begin{array}{c}\text { French \& } \\
\text { Belgian }\end{array}$} & \multicolumn{2}{|c|}{ Total cohort } \\
\hline & cases & controls & cases & controls & cases & controls & cases & controls & cases & controls \\
\hline $\begin{array}{l}\text { Sample } \\
\text { number }\end{array}$ & 3,777 & 33,365 & 2,853 & 2,143 & 449 & 226 & 1,150 & 595 & 8,229 & 36,329 \\
\hline $\begin{array}{r}\text { Female } \\
(\%)\end{array}$ & $\begin{array}{l}1,515 \\
(40.1)\end{array}$ & $\begin{array}{c}23,870 \\
(71.5)\end{array}$ & $\begin{array}{l}1,239 \\
(43.4)\end{array}$ & $\begin{array}{c}896 \\
(41.8)\end{array}$ & $\begin{array}{c}193 \\
(43.0)\end{array}$ & $\begin{array}{c}109 \\
(48.2)\end{array}$ & $\begin{array}{c}486 \\
(42.3)\end{array}$ & $\begin{array}{c}422 \\
(70.9)\end{array}$ & $\begin{array}{l}3,433 \\
(41.7)\end{array}$ & $\begin{array}{c}25,297 \\
(69.6)\end{array}$ \\
\hline $\begin{array}{l}\text { Age } \\
\text { (SD) }\end{array}$ & $\begin{array}{c}58.1 \\
(12.3)\end{array}$ & $\begin{array}{c}64.2 \\
(13.3)\end{array}$ & $\begin{array}{c}61.8 \\
(11.8)\end{array}$ & $\begin{array}{c}50.6 \\
(17.4)\end{array}$ & $\begin{array}{c}60.3 \\
(12.8)\end{array}$ & $\begin{array}{l}57.0 \\
(0.0)\end{array}$ & $\begin{array}{c}60.5 \\
(12.6)\end{array}$ & $\begin{array}{c}66.9 \\
(16.8)\end{array}$ & $\begin{array}{c}59.8 \\
(12.3)\end{array}$ & $\begin{array}{c}63.4 \\
(13.9)\end{array}$ \\
\hline $\begin{array}{r}\text { Bulbar-onset } * \\
(\%)\end{array}$ & $\begin{array}{c}963 \\
(25.5)\end{array}$ & - & $\begin{array}{c}741 \\
(26.0)\end{array}$ & - & $\begin{array}{c}141 \\
(31.4)\end{array}$ & - & $\begin{array}{l}357 \\
(31)\end{array}$ & - & $\begin{array}{l}2,202 \\
(26.8)\end{array}$ & - \\
\hline $\begin{array}{r}\text { Family } \\
\text { history }^{\dagger} \\
(\%)\end{array}$ & $\begin{array}{c}458 \\
(12.1)\end{array}$ & - & $\begin{array}{c}248 \\
(8.7)\end{array}$ & - & $\begin{array}{c}54 \\
(12.0)\end{array}$ & - & $\begin{array}{c}195 \\
(17.0)\end{array}$ & - & $\begin{array}{c}955 \\
(11.6)\end{array}$ & - \\
\hline
\end{tabular}

SD, standard deviation. *Data not available for site of symptom onset for 199 patients. $\uparrow$ Data not available for familial history of 154 patients. 
Table S2. Related to Figure 1; DbGaP studies contributing to the GWAS analysis.

\begin{tabular}{|c|c|c|c|c|c|c|}
\hline $\begin{array}{l}\text { Accession } \\
\text { Number }\end{array}$ & Study & $\begin{array}{l}\text { Sample } \\
\text { number }\end{array}$ & $\begin{array}{c}\text { Females } \\
(\%)\end{array}$ & $\begin{array}{l}\text { Average } \\
\text { age (SD) }\end{array}$ & $\begin{array}{l}\text { Genotyping } \\
\text { platform }\end{array}$ & $\begin{array}{l}\text { Ascertainment } \\
\text { criteria }\end{array}$ \\
\hline phs000001 & $\begin{array}{l}\text { NEI Age-Related Eye Disease Study } \\
\text { (AREDS) }\end{array}$ & 1,644 & $959(58.3)$ & $68.2(4.8)$ & HumanOmni2.5 & Population controls \\
\hline phs000007 & Framingham Cohort & 1,298 & $718(55.3)$ & $75.7(8.6)$ & HumanOmni5 & Population controls \\
\hline phs000187 & $\begin{array}{c}\text { High Density SNP Association Analysis } \\
\text { of Melanoma }\end{array}$ & 1,027 & $414(40.3)$ & $51.3(12.6)$ & HumanOmniExpress & Population controls \\
\hline phs000196 & $\begin{array}{l}\text { CIDR: The NeuroGenetics Research } \\
\text { Consortium Parkinson's Disease Study }\end{array}$ & 10 & $6(60)$ & $74.3(18.6)$ & HumanOmni1 & Population controls \\
\hline phs000292 & $\begin{array}{l}\text { GENEVA Genetics of Early Onset Stroke } \\
\text { (GEOS) Study }\end{array}$ & 89 & $0(0)$ & $41.5(6.4)$ & HumanOmni1 & Population controls \\
\hline phs000304 & Genes and Blood Clotting Study (GABC) & 403 & $259(64.3)$ & $21.6(3.3)$ & HumanOmni1 & Population controls \\
\hline phs000315 & $\begin{array}{l}\text { Woman's Health Initiative (WHI } \\
\text { GARNET) }\end{array}$ & 4,206 & $4206(100)$ & $65.7(6.9)$ & HumanOmni1 & Population controls \\
\hline phs000368 & $\begin{array}{c}\text { Polycystic Ovary Syndrome Genetics } \\
\text { (POLYGEN) }\end{array}$ & 2,974 & $2973(100)$ & $46.8(15.2)$ & HumanOmniExpress & Population controls \\
\hline phs000372 & $\begin{array}{l}\text { Alzheimer's Disease Genetics Consortium } \\
\text { Genome Wide Association Study }\end{array}$ & 533 & $335(62.9)$ & $75.8(9)$ & HumanOmniExpress & Population controls \\
\hline phs000394 & $\begin{array}{c}\text { Autopsy-Confirmed Parkinson Disease } \\
\text { GWAS Consortium (APDGC) }\end{array}$ & 299 & $152(50.8)$ & $82.1(12.6)$ & HumanOmni1 & Population controls \\
\hline phs000397 & NIA Long Life Family Study (LLFS) & 1,804 & $957(53)$ & $65.9(12.3)$ & HumanOmni2.5 & Population controls \\
\hline phs000404 & $\begin{array}{l}\text { The Genetic Architecture of Smoking and } \\
\text { Smoking Cessation }\end{array}$ & 81 & $50(61.7)$ & $36.6(5.9)$ & HumanOmni2.5 & Population controls \\
\hline phs000421 & $\begin{array}{l}\text { A Genome-Wide Association Study of } \\
\text { Fuchs' Endothelial Corneal Dystrophy }\end{array}$ & 497 & $294(59.2)$ & $70.4(10.2)$ & HumanOmni2.5 & Population controls \\
\hline phs000428 & Health and Retirement Study (HRS) & 9,394 & $5437(57.9)$ & $68.4(9.4)$ & HumanOmni2.5 & Population controls \\
\hline phs000615 & NINDS Stroke Genetics Network (SiGN) & 743 & $416(56)$ & $56(16.1)$ & HumanOmni5 & Population controls \\
\hline phs000675 & $\begin{array}{c}\text { GWAS on Selected WHI Hormone Trial } \\
\text { European Americans }\end{array}$ & 5,626 & $5626(100)$ & $68(5.9)$ & HumanOmni1 & Population controls \\
\hline phs000801 & NCI Non-Hodgkin Lymphoma GWAS & 1,544 & $790(51.2)$ & $58.4(11.6)$ & HumanOmniExpress & Population controls \\
\hline phs000869 & $\begin{array}{l}\text { Barrett's and Esophageal Adenocarcinoma } \\
\text { Genetic Susceptibility Study (BEAGESS) }\end{array}$ & 1,174 & $271(23.1)$ & $61.3(10.9)$ & HumanOmni1 & Population controls \\
\hline
\end{tabular}


Table S3. Related to Figure 1; SNPs achieving genome-wide significance in the GWAS analysis.

\begin{tabular}{|c|c|c|c|c|c|c|c|c|c|c|c|c|c|}
\hline \multicolumn{4}{|c|}{ SNP Information } & \multicolumn{3}{|c|}{$\begin{array}{c}\text { Present Study } \\
(8,229 \text { Cases / 36,329 Controls }) \\
\end{array}$} & \multicolumn{3}{|c|}{$\begin{array}{c}\text { Van Rheenen } \text { et al. } \\
(12,577 \text { Cases / 23,475 Controls) }\end{array}$} & \multicolumn{4}{|c|}{$\begin{array}{c}\text { Combined Discovery Set } \\
(20,806 \text { Cases / 59,804 Controls }) \\
\end{array}$} \\
\hline SNP & Chr & Position & Gene & $\begin{array}{l}\text { Beta } \\
\text { [SE] }\end{array}$ & $\begin{array}{c}\text { OR } \\
{[95 \% \mathrm{CI}]}\end{array}$ & $P$ & $\begin{array}{l}\text { Beta } \\
\text { [SE] }\end{array}$ & $\begin{array}{c}\text { OR } \\
{[95 \% \mathrm{CI}]}\end{array}$ & $P$ & $\mathbf{I}^{2}$ & $\begin{array}{l}\text { Beta } \\
\text { [SE] }\end{array}$ & $\begin{array}{c}\text { OR } \\
{[95 \% \mathrm{CI}]}\end{array}$ & $\boldsymbol{P}$ \\
\hline \multicolumn{14}{|l|}{ Novel Loci } \\
\hline rs117027576 & 12 & $57,316,603$ & KIF5A & $\begin{array}{c}0.373 \\
{[0.096]}\end{array}$ & $\begin{array}{c}1.45 \\
{[1.20-1.76]}\end{array}$ & $1.1 \times 10^{-4}$ & $\begin{array}{c}0.286 \\
{[0.070]}\end{array}$ & $\begin{array}{c}1.33 \\
{[1.16-1.53]}\end{array}$ & $4.3 \times 10^{-5}$ & 25.6 & $\begin{array}{c}0.316 \\
{[0.057]}\end{array}$ & $\begin{array}{c}1.37 \\
{[1.23-1.54]}\end{array}$ & $2.3 \times 10^{-8}$ \\
\hline rs118082508 & 12 & $57,318,819$ & KIF5A & $\begin{array}{c}0.374 \\
{[0.096]}\end{array}$ & $\begin{array}{c}1.45 \\
{[1.20-1.76]}\end{array}$ & $1.0 \times 10^{-4}$ & $\begin{array}{c}0.288 \\
{[0.070]}\end{array}$ & $\begin{array}{c}1.33 \\
{[1.16-1.53]}\end{array}$ & $3.8 \times 10^{-5}$ & 25.8 & $\begin{array}{c}0.317 \\
{[0.051]}\end{array}$ & $\begin{array}{c}1.37 \\
{[1.23-1.54]}\end{array}$ & $2.0 \times 10^{-8}$ \\
\hline rs113247976* & 12 & $57,975,700$ & KIF5A & $\begin{array}{c}0.381 \\
{[0.086]}\end{array}$ & $\begin{array}{c}1.46 \\
{[1.23-1.74]}\end{array}$ & $9.2 \times 10^{-6}$ & $\begin{array}{c}0.288 \\
{[0.066]}\end{array}$ & $\begin{array}{c}1.33 \\
{[1.17-1.52]}\end{array}$ & $1.1 \times 10^{-5}$ & 0.0 & $\begin{array}{c}0.322 \\
{[0.052]}\end{array}$ & $\begin{array}{c}1.38 \\
{[1.24-1.53]}\end{array}$ & $6.4 \times 10^{-10}$ \\
\hline rs116900480 & 12 & $58,656,105$ & KIF5A & $\begin{array}{c}0.354 \\
{[0.083]}\end{array}$ & $\begin{array}{c}1.42 \\
{[1.21-1.68]}\end{array}$ & $1.9 \times 10^{-5}$ & $\begin{array}{c}0.294 \\
{[0.065]}\end{array}$ & $\begin{array}{c}1.34 \\
{[1.18-1.53]}\end{array}$ & $7.1 \times 10^{-6}$ & 0.0 & $\begin{array}{c}0.317 \\
{[0.051]}\end{array}$ & $\begin{array}{c}1.37 \\
{[1.24-1.52]}\end{array}$ & $6.6 \times 10^{-10}$ \\
\hline rs142321490 & 12 & $58,676,132$ & KIF5A & $\begin{array}{c}0.357 \\
{[0.082]}\end{array}$ & $\begin{array}{c}1.43 \\
{[1.21-1.68]}\end{array}$ & $1.5 \times 10^{-5}$ & $\begin{array}{c}0.292 \\
{[0.066]}\end{array}$ & $\begin{array}{c}1.34 \\
{[1.18-1.53]}\end{array}$ & $8.0 \times 10^{-6}$ & 0.0 & $\begin{array}{c}0.317 \\
{[0.056]}\end{array}$ & $\begin{array}{c}1.37 \\
{[1.24-1.52]}\end{array}$ & $6.1 \times 10^{-10}$ \\
\hline \multicolumn{14}{|c|}{ Previously Published Loci } \\
\hline rs10463311 & 5 & $150,410,835$ & TNIP1 & $\begin{array}{l}-0.065 \\
{[0.024]}\end{array}$ & $\begin{array}{c}0.94 \\
{[0.89-0.98]}\end{array}$ & $7.8 \times 10^{-3}$ & $\begin{array}{l}-0.100 \\
{[0.020]}\end{array}$ & $\begin{array}{c}0.91 \\
{[0.87-0.94]}\end{array}$ & $8.5 \times 10^{-7}$ & 0.0 & $\begin{array}{l}-0.085 \\
{[0.016]}\end{array}$ & $\begin{array}{c}0.92 \\
{[0.89-0.95]}\end{array}$ & $4.0 \times 10^{-8}$ \\
\hline rs3849943 & 9 & $27,543,382$ & C9orf72 & $\begin{array}{l}-0.17 \\
{[0.024]}\end{array}$ & $\begin{array}{c}0.84 \\
{[0.80-0.88]}\end{array}$ & $1.4 \times 10^{-12}$ & $\begin{array}{c}-0.181 \\
{[0.020]}\end{array}$ & $\begin{array}{c}0.83 \\
{[0.80-0.87]}\end{array}$ & $4.0 \times 10^{-19}$ & 0.0 & $\begin{array}{c}-0.176 \\
{[0.016]}\end{array}$ & $\begin{array}{c}0.84 \\
{[0.81-0.86]}\end{array}$ & $3.8 \times 10^{-30}$ \\
\hline rs74654358 & 12 & $64,881,967$ & $T B K 1$ & $\begin{array}{c}0.182 \\
{[0.058]}\end{array}$ & $\begin{array}{c}1.20 \\
{[1.07-1.34]}\end{array}$ & $1.6 \times 10^{-3}$ & $\begin{array}{c}0.206 \\
{[0.042]}\end{array}$ & $\begin{array}{c}1.23 \\
{[1.13-1.34]}\end{array}$ & $7.7 \times 10^{-7}$ & 0.0 & $\begin{array}{c}0.198 \\
{[0.034]}\end{array}$ & $\begin{array}{c}1.22 \\
{[1.14-1.30]}\end{array}$ & $4.7 \times 10^{-9}$ \\
\hline rs12973192 & 19 & $17,753,239$ & $U N C 13 A$ & $\begin{array}{c}-0.149 \\
{[0.026]}\end{array}$ & $\begin{array}{c}0.86 \\
{[0.82-0.91]}\end{array}$ & $1.3 \times 10^{-8}$ & $\begin{array}{c}-0.106 \\
{[0.019]}\end{array}$ & $\begin{array}{c}0.9 \\
{[0.87-0.93]}\end{array}$ & $2.4 \times 10^{-8}$ & 38.6 & $\begin{array}{c}-0.121 \\
{[0.015]}\end{array}$ & $\begin{array}{c}0.89 \\
{[0.86-0.91]}\end{array}$ & $3.9 \times 10^{-15}$ \\
\hline rs75087725 & 21 & $45,753,117$ & C21orf2 & $\begin{array}{c}0.687 \\
{[0.162]}\end{array}$ & $\begin{array}{c}1.99 \\
{[1.44-2.75]}\end{array}$ & $2.2 \times 10^{-5}$ & $\begin{array}{c}0.479 \\
{[0.074]}\end{array}$ & $\begin{array}{c}1.61 \\
{[1.39-1.87]}\end{array}$ & $8.7 \times 10^{-11}$ & 31.1 & $\begin{array}{c}0.515 \\
{[0.067]}\end{array}$ & $\begin{array}{c}1.67 \\
{[1.46-1.91]}\end{array}$ & $1.8 \times 10^{-14}$ \\
\hline
\end{tabular}

Position is based on Human Genome Assembly build 37. Nearest gene or previously published gene names are included. Chr, chromosome; SE, standard error; OR, odds ratio; 95\% CI, 95\% confidence interval; *, rs113247976 represents the p.Pro986Leu variant in KIF5A (NM_004984.2). 
Table S4. Related to Figure 1; Suggestive SNPs with $P$ values less than 5.0x10-7 in the GWAS analyses.

\begin{tabular}{|c|c|c|c|c|c|c|c|c|c|c|c|c|c|c|c|}
\hline \multicolumn{4}{|c|}{ SNP Information } & \multicolumn{4}{|c|}{$\begin{array}{c}\text { Present Study } \\
(8,229 \text { Cases / 36,329 Controls })\end{array}$} & \multicolumn{4}{|c|}{$\begin{array}{c}\text { Van Rheenen } \text { et al. } \\
(12,577 \text { Cases / 23,475 Controls) }\end{array}$} & \multicolumn{4}{|c|}{$\begin{array}{c}\text { Combined Discovery Set } \\
(20,806 \text { Cases / 59,804 Controls) }\end{array}$} \\
\hline SNP & Chr & Position & Gene & $\begin{array}{l}\text { Case } \\
\text { MAF } \\
\end{array}$ & $\begin{array}{c}\text { Control } \\
\text { MAF } \\
\end{array}$ & $\begin{array}{c}\text { OR } \\
{[95 \% \mathrm{CI}]}\end{array}$ & $P$ & $\begin{array}{l}\text { Case } \\
\text { MAF } \\
\end{array}$ & $\begin{array}{l}\text { Control } \\
\text { MAF }\end{array}$ & $\begin{array}{c}\text { OR } \\
{[95 \% \mathrm{CI}]}\end{array}$ & $P$ & $\begin{array}{l}\text { Case } \\
\text { MAF }\end{array}$ & $\begin{array}{l}\text { Control } \\
\text { MAF }\end{array}$ & $\begin{array}{c}\text { OR } \\
{[95 \% \mathrm{CI}]}\end{array}$ & $P$ \\
\hline rs17070492 & 8 & $2,420,855$ & LOC101927815 & $10.01 \%$ & $9.76 \%$ & $\begin{array}{c}1.10 \\
{[1.02-1.18]}\end{array}$ & $1.3 \times 10^{-2}$ & $9.17 \%$ & $10.09 \%$ & $\begin{array}{c}1.16 \\
{[1.09-1.23]}\end{array}$ & $1.3 \times 10^{-6}$ & $9.50 \%$ & $9.89 \%$ & $\begin{array}{c}1.13 \\
{[1.08-1.19]}\end{array}$ & $1.0 \times 10^{-7}$ \\
\hline rs10139154 & 14 & $31,147,498$ & SCFD1 & $34.10 \%$ & $31.30 \%$ & $\begin{array}{c}1.07 \\
{[1.03-1.12]}\end{array}$ & $2.1 \times 10^{-3}$ & $33.76 \%$ & $31.17 \%$ & $\begin{array}{c}1.08 \\
{[1.04-1.12]}\end{array}$ & $1.9 \times 10^{-5}$ & $33.90 \%$ & $31.25 \%$ & $\begin{array}{c}1.08 \\
{[1.05-1.11]}\end{array}$ & $1.4 \times 10^{-7}$ \\
\hline rs 10143310 & 14 & $92,540,381$ & ATXN3 & $24.85 \%$ & $24.36 \%$ & $\begin{array}{c}1.09 \\
{[1.04-1.015]}\end{array}$ & $3.3 \times 10^{-4}$ & $24.04 \%$ & $22.95 \%$ & $\begin{array}{c}1.08 \\
{[1.04-1.13]}\end{array}$ & $2.6 \times 10^{-4}$ & $24.36 \%$ & $23.81 \%$ & $\begin{array}{c}1.09 \\
{[1.05-1.12]}\end{array}$ & $3.2 \times 10^{-7}$ \\
\hline rs9901522 & 17 & $14,673,934$ & $P M P 22$ & $7.08 \%$ & $6.31 \%$ & $\begin{array}{c}1.16 \\
{[1.06-1.26]}\end{array}$ & $5.2 \times 10^{-4}$ & $6.87 \%$ & $5.97 \%$ & $\begin{array}{c}1.16 \\
{[1.08-1.24]}\end{array}$ & $4.6 \times 10^{-5}$ & $6.95 \%$ & $6.18 \%$ & $\begin{array}{c}1.16 \\
{[1.10-1.22]}\end{array}$ & $8.6 \times 10^{-8}$ \\
\hline
\end{tabular}


Table S5. Related to Figure 3; DbGaP/EGA studies contributing to the RVB analysis.

\begin{tabular}{|c|c|c|c|}
\hline Accession Number & Study & $\begin{array}{l}\text { Sample } \\
\text { number }\end{array}$ & $\begin{array}{c}\text { Females } \\
(\%)\end{array}$ \\
\hline phs000179 & Genetic Epidemiology of COPD (COPDGene) & 2 & $100 \%$ \\
\hline phs000254 & NHLBI GO-ESP: Lung Cohorts Exome Sequencing Project (Cystic Fibrosis) & 238 & $49.6 \%$ \\
\hline phs000281 & NHLBI GO-ESP: Women's Health Initiative Exome Sequencing Project (WHI) - WHISP & 1904 & $100 \%$ \\
\hline phs000290 & NHLBI GO-ESP: Lung Cohorts Exome Sequencing Project (Pulmonary Arterial Hypertension) & 73 & $82.2 \%$ \\
\hline phs000291 & NHLBI GO-ESP: Lung Cohorts Exome Sequencing Project (Lung Health Study of COPD) & 332 & $37 \%$ \\
\hline phs000296 & NHLBI GO-ESP: Lung Cohorts Exome Sequencing Project (COPDGene) & 285 & $52.6 \%$ \\
\hline phs000307 & NHLBI Framingham Heart Study Allelic Spectrum Project & 1317 & $51.6 \%$ \\
\hline phs000347 & NHLBI GO-ESP: Family Studies (Aortic Disease) & 29 & $34.5 \%$ \\
\hline phs000354 & NHLBI GO-ESP Family Studies: Pulmonary Arterial Hypertension & 9 & $88.9 \%$ \\
\hline phs000362 & NHLBI GO-ESP: Family Studies: (Familial Atrial Fibrillation) & 12 & $16.7 \%$ \\
\hline phs000398 & NHLBI GO-ESP: Heart Cohorts Exome Sequencing Project (ARIC) & 800 & $54.6 \%$ \\
\hline phs000400 & NHLBI GO-ESP: Heart Cohorts Exome Sequencing Project (CHS) & 186 & $28 \%$ \\
\hline phs000401 & NHLBI GO-ESP: Heart Cohorts Exome Sequencing Project (FHS) & 348 & $36.8 \%$ \\
\hline phs000402 & NHLBI GO-ESP: Heart Cohorts Exome Sequencing Project (JHS) & 296 & $58.8 \%$ \\
\hline phs000403 & NHLBI GO-ESP: Heart Cohorts Exome Sequencing Project (MESA) & 259 & $45.2 \%$ \\
\hline phs000422 & NHLBI GO-ESP: Lung Cohorts Exome Sequencing Project (Asthma) & 189 & $65.1 \%$ \\
\hline phs000498 & Jackson Heart Study Allelic Spectrum Project & 1629 & $63.8 \%$ \\
\hline phs000518 & NHLBI GO-ESP Family Studies: Idiopathic Bronchiectasis & 24 & $70.8 \%$ \\
\hline phs000572 & Alzheimer's Disease Sequencing Project (ADSP) & 4655 & $58.8 \%$ \\
\hline phs000632 & NHLBI GO-ESP: Family Studies (Hematological Cancers) & 19 & $36.8 \%$ \\
\hline phs000651 & Building on GWAS: the U.S. CHARGE consortium - Sequencing (CHARGE-S): FHS & 550 & $61.5 \%$ \\
\hline phs000667 & Building on GWAS for NHLBI-Diseases: The U.S. CHARGE Consortium - Sequencing (CHARGE-S): CHS & 1209 & $52.9 \%$ \\
\hline phs000668 & Building on GWAS: the U.S. CHARGE consortium - Sequencing (CHARGE-S): ARIC & 5497 & $58.5 \%$ \\
\hline phs000744 & Yale Center for Mendelian Genomics (Y CMG) & 1944 & $44.7 \%$ \\
\hline phs000806 & MIGen_ExS: Ottawa Heart Study & 1966 & $33.1 \%$ \\
\hline phs000814 & MIGen_ExS: Italian Atherosclerosis Thrombosis and Vascular Biology & 3591 & $11.3 \%$ \\
\hline phs000908 & Identification of Rare Variants in PD through Whole Exome Sequencing & 105 & $66.7 \%$ \\
\hline phs000917 & MIGen_ExS: PROMIS & 7298 & $17.9 \%$ \\
\hline phs001000 & MIGen_ExS: U. of Leicester & 1081 & $0 \%$ \\
\hline phs001101 & MIGen_ExS: MDC & 1075 & $44.7 \%$ \\
\hline EGAO00000000079 & UK10K & 4062 & $65 \%$ \\
\hline phs000101 & NIH Exome Sequencing of Familial Amyotrophic Lateral Sclerosis Project & 201 & $45 \%$ \\
\hline
\end{tabular}


Table S6. Related to Figure 2, 3; Quality control filtering of the FALS discovery and KIF5A replication cohorts.

FALS discovery cohort

\begin{tabular}{ccc}
\hline Cohort & Cases & Controls \\
\hline Initial Sample Set & 1,463 & 41,410 \\
Post HGDP Continental Ancestry Filter & 1,397 & 24,563 \\
Post Call Rate Filter & 1,331 & 20,789 \\
Post Heterozygosity Filter & 1,319 & 20,664 \\
Post Relatedness Filter & 1,138 & 19,494 \\
\hline
\end{tabular}

rs113247976 replication cohort (FALS discovery + ALS WXS/WGS replication cohort)

\begin{tabular}{ccc}
\hline Cohort & Cases & Controls \\
\hline Initial Sample Set & $12,180^{*}$ & $21,533^{* *}$ \\
Post Call Rate Filter & 11,916 & 21,050 \\
Post Heterozygosity Filter & 11,721 & 21,028 \\
Post Ancestry Filter (PCA) & 11,373 & 21,009 \\
Post Relatedness \& GWAS Checksum Filter & 4,160 & 18,650 \\
\hline * All 1,138 FALS passing QC in FALS discovery cohort + 11,042 additional ALS WXS/WGS cases \\
** All 19,494 controls passing QC in FALS discovery cohort + 2,039 additional WXS/WGS controls \\
LOF screen (ALS WXS/WGS replication cohort) & \\
\hline Cohort & Cases & \\
\hline Initial Sample Set & $11,042^{*}$ & $2,039^{* *}$ \\
Post Call Rate Filter & 10,741 & 2,039 \\
Post Heterozygosity Filter & 10,549 & 2,026 \\
Post Ancestry Filter (PCA) & 10,201 & 2,008 \\
Post Relatedness & 9,046 & 1,955 \\
\hline
\end{tabular}

* 11,042 additional ALS WXS/WGS cases not included in FALS discovery cohort

** 2,039 additional WXS/WGS controls not included in FALS discovery cohort

See Experimental Procedures for further details on filtering parameters. 
Table S7. Related to Figure 3; RVB analysis according to mutation type across KIF5A and within gene sub-domains.

\begin{tabular}{|c|c|c|c|c|}
\hline Analysis & FALS & Control & OR $(95 \% \mathrm{CI})$ & $\boldsymbol{P}$ \\
\hline Missense - Full CDS & $9(0.79 \%)$ & $80(0.41 \%)$ & $1.93(0.915-3.60)$ & $8.09 \times 10^{-2}$ \\
\hline Missense - Motor Domain & $3(0.26 \%)$ & $18(0.09 \%)$ & $3.27(0.86-9.25)$ & $7.74 \times 10^{-2}$ \\
\hline Missense - Microtubule Binding Domain & $2(0.18 \%)$ & $8(0.04 \%)$ & $5.07(0.95-18.52)$ & $5.57 \times 10^{-2}$ \\
\hline Missense - Coiled-Coil Domain & $3(0.26 \%)$ & $55(0.28 \%)$ & $1.01(0.28-2.60)$ & $9.83 \times 10^{-1}$ \\
\hline Missense - C-Terminal Domain & $3(0.26 \%)$ & $7(0.04 \%)$ & $7.23(1.74-24.55)$ & $9.41 \times 10^{-3}$ \\
\hline Loss of Function & $6(0.53 \%)$ & $3(0.02 \%)$ & $32.07(9.05-135.27)$ & $5.55 \times 10^{-7}$ \\
\hline Loss of Function (including frameshifts) & $8(0.70 \%)$ & $3(0.02 \%)$ & $41.16(12.61-167.57)$ & $3.77 \times 10^{-9}$ \\
\hline
\end{tabular}

FALS, familial ALS; OR, odds ratio; 95\% CI, 95\% confidence interval; CDS, coding sequence 
Table S8. Related to Figure 3; Clinical information of probands and relatives carrying KIF5A LOF variants.

\begin{tabular}{|c|c|c|c|c|c|c|c|c|c|c|c|}
\hline Position & Variant & $\begin{array}{c}\text { Relation to } \\
\text { Proband }\end{array}$ & $\begin{array}{c}\text { DNA } \\
\text { Available }\end{array}$ & Exon & cDNA & Description & Gender & $\begin{array}{c}\text { Age of Onset } \\
\text { (years) }\end{array}$ & $\begin{array}{l}\text { Site of } \\
\text { Onset }\end{array}$ & $\begin{array}{l}\text { Survival } \\
\text { (months) }\end{array}$ & Alive \\
\hline $57,975,729$ & $\mathrm{GA}>\mathrm{A}$ & Proband & $\mathrm{Y}$ & 26 & c. $2987 \mathrm{delA}$ & p.Asp996fs & $\mathrm{M}$ & 45 & $\mathrm{n} / \mathrm{a}$ & $\mathrm{n} / \mathrm{a}$ & $\mathrm{n} / \mathrm{a}$ \\
\hline $57,976,382$ & $\mathrm{C}>\mathrm{T}$ & Proband & $\mathrm{Y}$ & 27 & c. $2993-3 C>T$ & 5' Splice Junction & M & 29 & $\mathrm{~L}$ & $>264$ & $\mathrm{Y}$ \\
\hline $57,976,382$ & $\mathrm{C}>\mathrm{T}$ & Sister & $\mathrm{Y}$ & 27 & c. $2993-3 \mathrm{C}>\mathrm{T}$ & 5' Splice Junction & $\mathrm{F}$ & 52 & $\mathrm{~L}$ & 84 & $\mathrm{~N}$ \\
\hline \multirow[t]{2}{*}{$57,976,382$} & $\mathrm{C}>\mathrm{T}$ & Brother & $\mathrm{Y}$ & 27 & c. $2993-3 C>T$ & 5' Splice Junction & M & 18 & $\mathrm{~L}$ & 324 & $\mathrm{~N}$ \\
\hline & & Brother & $\mathrm{N}$ & & & & M & $\mathrm{n} / \mathrm{a}$ & $\mathrm{L}$ & $\mathrm{n} / \mathrm{a}$ & $\mathrm{N}$ \\
\hline $57,975,731$ & $\mathrm{CA}>\mathrm{C}$ & Sporadic & $\mathrm{Y}$ & 26 & c. $2989 \mathrm{delA}$ & p.Asn997fs & $\mathrm{F}$ & 50 & $\mathrm{~L}$ & $>96$ & $\mathrm{Y}$ \\
\hline $57,976,384$ & $\mathrm{G}>\mathrm{A}$ & Sporadic & $\mathrm{N}$ & 27 & c. $2993-1 \mathrm{G}>\mathrm{A}$ & 5' Splice Junction & $\mathrm{n} / \mathrm{a}$ & 52 & B & $\mathrm{n} / \mathrm{a}$ & $\mathrm{n} / \mathrm{a}$ \\
\hline \multirow[t]{2}{*}{$57,976,385$} & $\mathrm{GA}>\mathrm{G}$ & Proband & $\mathrm{Y}$ & 27 & c. $2996 \mathrm{delA}$ & p.Asn999fs & M & 42 & $\mathrm{~L}$ & $>12$ & $\mathrm{Y}$ \\
\hline & & Brother & $\mathrm{N}$ & & & & M & 38 & $\mathrm{n} / \mathrm{a}$ & 24 & $\mathrm{~N}$ \\
\hline $57,976,411$ & $\mathrm{~A}>\mathrm{G}$ & Proband & $\mathrm{Y}$ & 27 & c. $3019 A>G$ & p.Arg 1007Gly & $\mathrm{F}$ & 53 & $\mathrm{~L}$ & 45 & $\mathrm{~N}$ \\
\hline $57,976,412$ & $\mathrm{G}>\mathrm{A}$ & Proband & $\mathrm{Y}$ & 27 & c. $3020 \mathrm{G}>\mathrm{A}$ & p.Arg 1007Lys & M & 50 & $\mathrm{~L}$ & $>108$ & $\mathrm{Y}$ \\
\hline $57,976,412$ & $\mathrm{G}>\mathrm{A}$ & Proband & $\mathrm{Y}$ & 27 & c. $3020 \mathrm{G}>\mathrm{A}$ & p.Arg 1007Lys & $\mathrm{F}$ & 50 & $\mathrm{n} / \mathrm{a}$ & $>240$ & $\mathrm{Y}$ \\
\hline \multirow[t]{5}{*}{$57,976,413$} & $\mathrm{G}>\mathrm{A}$ & Proband & $\mathrm{Y}$ & 27 & c. $3020+1 \mathrm{G}>\mathrm{A}$ & 3' Splice Junction & M & 45 & B & $>220$ & $\mathrm{Y}$ \\
\hline & & Parent & $\mathrm{N}$ & & & & $\mathrm{n} / \mathrm{a}$ & 47 & $\mathrm{n} / \mathrm{a}$ & 156 & $\mathrm{~N}$ \\
\hline & & Uncle/Aunt & $\mathrm{N}$ & & & & $\mathrm{n} / \mathrm{a}$ & 57 & $\mathrm{n} / \mathrm{a}$ & 144 & $\mathrm{~N}$ \\
\hline & & Uncle/Aunt & $\mathrm{N}$ & & & & $\mathrm{n} / \mathrm{a}$ & 55 & $\mathrm{n} / \mathrm{a}$ & 121 & $\mathrm{~N}$ \\
\hline & & Uncle/Aunt & $\mathrm{N}$ & & & & $\mathrm{n} / \mathrm{a}$ & 46 & $\mathrm{n} / \mathrm{a}$ & 24 & $\mathrm{~N}$ \\
\hline $57,976,414$ & $\mathrm{~T}>\mathrm{A}$ & Proband & $\mathrm{Y}$ & 27 & c. $3020+2 \mathrm{~T}>\mathrm{A}$ & 3' Splice Junction & M & 46 & B & 124 & $\mathrm{~N}$ \\
\hline \multirow[t]{2}{*}{$57,976,414$} & $\mathrm{~T}>\mathrm{A}$ & Brother & $\mathrm{Y}$ & 27 & c. $3020+2 \mathrm{~T}>\mathrm{A}$ & 3' Splice Junction & M & 48 & $\mathrm{~L}$ & 117 & $\mathrm{~N}$ \\
\hline & & Mother & $\mathrm{N}$ & & & & $\mathrm{F}$ & 35 & $\mathrm{~L}$ & 144 & $\mathrm{~N}$ \\
\hline $57,976,415$ & $\mathrm{~A}>\mathrm{G}$ & Proband & $\mathrm{Y}$ & 27 & c. $3020+3 A>G$ & 3' Splice Junction & M & 50 & B & 54 & $\mathrm{~N}$ \\
\hline
\end{tabular}

All mutations were heterozygous; Genomic coordinates are based on Human Genome Assembly build 37; Protein change is based on transcript NM_004984.3; n/a, not applicable or not available 


\section{CONSORTIUM MEMBERS}

\section{ALS Sequencing Consortium}

Andrew S. Allen ${ }^{1}$, Stanley Appel ${ }^{2}$, Robert H. Baloh ${ }^{3}$, Richard S. Bedlack ${ }^{4}$, Braden E. Boone $^{5}$, Robert Brown ${ }^{6}$, John P. Carulli ${ }^{7}$, Alessandra Chesi ${ }^{8}$, Wendy K. Chung ${ }^{9}$, Elizabeth T. Cirulli ${ }^{10}$, Gregory M. Cooper ${ }^{5}$, Julien Couthouis ${ }^{8}$, Aaron G. Day-Williams ${ }^{7}$, Patrick A. Dion ${ }^{11}$, Summer Gibson ${ }^{12}$, Aaron D. Gitler ${ }^{8}$, Jonathan D. Glass ${ }^{13}$, David B. Goldstein $^{14}$, Yujun Han ${ }^{15}$, Matthew B. Harms ${ }^{16}$, Tim Harris $^{17}$, Sebastian D. Hayes ${ }^{18}$, Angela L. Jones ${ }^{5}$, Jonathan Keebler ${ }^{15}$, Brian J. Krueger ${ }^{14}$, Brittany N. Lasseigne ${ }^{5}$, Shawn E. Levy ${ }^{5}$, Yi-Fan Lu ${ }^{14}$, Tom Maniatis ${ }^{19}$, Diane McKenna-Yasek ${ }^{6}$, Timothy M. Miller ${ }^{20}$, Richard M. Myers ${ }^{5}$, Slavé Petrovski ${ }^{14}$, Stefan M. Pulst ${ }^{12}$, Alya R. Raphael ${ }^{8}$, John M. Ravits $^{21}$, Zhong Ren ${ }^{14}$, Guy A. Rouleau ${ }^{11}$, Peter C. Sapp ${ }^{6}$, Neil A. Shneider ${ }^{22}$, Ericka Simpson ${ }^{2}$, Katherine B. Sims ${ }^{7}$, John F. Staropoli ${ }^{7}$, Lindsay L. Waite ${ }^{5}$, Quanli Wang ${ }^{14}$, Jack R. Wimbish ${ }^{5}$, Winnie W. Xin ${ }^{23}$

1. Department of Biostatistics and Bioinformatics, Duke University School of Medicine, Durham, NC 27708 USA.

2. Stanley Appel Department of Neurology, Houston Methodist, Houston,TX USA.

3. Department of Neurology, Cedars Sinai Medical Center, Los Angeles, CA 90048 USA.

4. Duke ALS Clinic and Durham VA Medical Center, Duke University School of Medicine, Durham, NC 27708 USA.

5. HudsonAlpha Institute for Biotechnology, Huntsville, AL 35806 USA.

6. Department of Neurology, University of Massachusetts Medical School, Worcester, MA 01655 USA.

7. Biogen Idec, Cambridge, MA 02142 USA.

8. Department of Genetics, Stanford University School of Medicine, Stanford, CA 94305 USA.

9. Department of Pediatrics and Medicine, College of Physicians and Surgeons, Columbia University, New York NY 10032

10. Human Longevity, INC, San Diego, CA 92121 USA.

11. Department of Neurology and Neurosurgery, McGill University, Montreal, H3A 2B4 Canada

12. Department of Neurology, University of Utah School of Medicine, Salt Lake City, UT 84112 USA.

13. Department of Neurology, Emory University, Atlanta, GA 30322 USA.

14. Institute for Genomic Medicine, Columbia University, New York, NY 10032 USA.

15. Duke University School of Medicine, Duke University School of Medicine, Durham, NC 27708 USA.

16. Department of Neurology/Institute for Genomic Medicine, Columbia University, New York, NY 11032 USA.

17. Bioverativ, 225 2nd Ave, Waltham, MA 02145

18. Biogen Idec and Harvard Medical School, Cambridge, MA 02142 USA.

19. Department of Biochemistry \& Molecular Biophysics, Columbia University, New York, NY 10027 USA. 
20. Department of Neurology, Washington University School of Medicine, St. Louis, MO 63110 USA.

21. Department of Neurosciences, University of California, San Diego, La Jolla CA 92093

22. Department of Neurology/Eleanor and Lou Gehrig ALS Center, Columbia University, New York, NY 10032 USA.

23. Neurogenetics DNA Diagnostic Laboratory, Center for Human Genetics Research Massachusetts General Hospital, Boston, MA 02114 USA.

\section{Answer ALS Foundation}

Julia Kaye ${ }^{1,}$ Steven Finkbeiner ${ }^{1,2}$,Stacia Wyman ${ }^{3,}$ Alexander LeNail ${ }^{4}$ Leandro Lima ${ }^{1,}$ Ernest Fraenkel $^{4,5,}$ Jeffrey D Rothstein ${ }^{6,}$ Clive N Svendsen ${ }^{7,8,}$ Leslie M Thompson ${ }^{9,10,}$ Jenny Van Eyk ${ }^{11,}$ Nicholas J Maragakis ${ }^{6,}$ James D Berry ${ }^{12,13,}$ Jonathan D Glass ${ }^{14,}$ Timothy M Miller $^{15,}$ Stephen J Kolb ${ }^{16,17}$, Robert H Baloh ${ }^{18,}$ Merit Cudkowicz ${ }^{19,20,}$ Emily Baxi $^{6}$

1. Gladstone Institute of Neurological Disease, San Francisco, CA, USA

2. Departments of Neurology and Physiology, University of California, San Francisco, CA, USA

3. Gladstone Institutes, San Francisco CA 94158

4. Department of Biological Engineering, Massachusetts Institute of Technology, 77 Massachusetts Avenue, Cambridge, Massachusetts, 02139, USA

5. Broad Institute, 415 Main St, Cambridge, Massachusetts, 02142, USA

6. Department of Neurology, Johns Hopkins University, Baltimore, MD 21287, USA

7. Board of Governors Regenerative Medicine Institute, Cedars-Sinai Medical Center, Los Angeles, CA, 90048, USA

8. Department of Biomedical Sciences, Cedars-Sinai Medical Center, Los Angeles, CA, 90048, USA

9. Department of Neurobiology and Behavior, Institute of Memory Impairment and Neurological Disorders, University of California, Irvine, CA 92697, USA

10. Department of Psychiatry and Human Behavior, Institute of Memory Impairment and Neurological Disorders, University of California, Irvine, CA 92697, USA.

11. The Heart Institute and Department of Medicine, Cedars-Sinai Medical Center, Los Angeles, CA

12. Harvard Medical School, Department of Neurology, Massachusetts General Hospital (MGH), Boston, Massachusetts, USA

13. Neurological Clinical Research Institute (NCRI), Massachusetts General Hospital, Boston, Massachusetts, USA

14. Department of Neurology, Emory University School of Medicine, Atlanta, GA

15. Department of Neurology, Washington University School of Medicine, St. Louis, MO, USA

16. Department of Biological Chemistry \& Pharmacology, The Ohio State University Wexner Medical Center, Columbus, $\mathrm{OH}$, United States

17. Department of Neurology, The Ohio State University Wexner Medical Center, Columbus, $\mathrm{OH}$, United States 
18. Department of Neurology, Cedars-Sinai Medical Center, Los Angeles, California, USA

19. Harvard Medical School, Department of Neurology, Massachusetts General Hospital (MGH), Boston, Massachusetts, USA.

20. Neurological Clinical Research Institute (NCRI), Massachusetts General Hospital, Boston, Massachusetts, USA

\section{Clinical Research in ALS and Related Disorders for Therapeutic Development (CReATe) Consortium}

Michael Benatar ${ }^{1}$, J. Paul Taylor ${ }^{2,3}$, Gang Wu ${ }^{4}$, Evadnie Rampersaud ${ }^{4}$, Joanne Wuu ${ }^{1}$, Rosa Rademakers ${ }^{5}$, Stephan Züchner ${ }^{6}$, Rebecca Schule ${ }^{7}$, Jacob McCauley ${ }^{6}$, Sumaira Hussain ${ }^{1}$, Anne Cooley ${ }^{1}$, Marielle Wallace ${ }^{1}$, Christine Clayman ${ }^{1}$, Richard Barohn ${ }^{8}$, Jeffrey Statland $^{8}$, John Ravits ${ }^{9}$, Andrea Swenson ${ }^{10}$, Carlayne Jackson ${ }^{11}$, Jaya Trivedi ${ }^{12}$, Shaida Khan $^{12}$, Jonathan Katz ${ }^{13}$, Liberty Jenkins ${ }^{13}$, Ted Burns ${ }^{14}$, Kelly Gwathmey ${ }^{14}$, James Caress $^{15}$, Corey McMillan ${ }^{16}$, Lauren Elman ${ }^{16}$, Erik Pioro ${ }^{17}$, Jeannine Heckmann ${ }^{18}$, Yuen So $^{19}$, David Walk ${ }^{20}$, Samuel Maiser ${ }^{21}$, Jinghui Zhang ${ }^{4}$

1. Department of Neurology, University of Miami

2. Howard Hughes Medical Institute

3. Department of Cell and Molecular Biology, St. Jude Children's Research Hospital

4. Department of Computational Biology, St. Jude Children's Research Hospital

5. Department of Neuroscience, Mayo Clinic Jacksonville

6. Department of Human Genetics, University of Miami

7. University of Tubingen, Hertie Institute for Clinical Brain Research

8. Department of Neurology, University of Kansas Medical Center

9. Department of Neurosciences, University of California San Diego

10. Department of Neurology, University of Iowa

11. Department of Neurology, University of Texas Health Sciences Center San Antonio

12. Department of Neurology \& Neurotherapeutics, University of Texas Southwestern Medical Center

13. Department of Neurology, California Pacific Medical Center

14. Department of Neurology, University of Virginia

15. Department of Neurology, Wake Forest University

16. Department of Neurology, University of Pennsylvania

17. Neuromuscular Center, Cleveland Clinic Ohio

18. Department of Neurology, University of Cape Town

19. Department of Neurology, Stanford University

20. Department of Neurology, University of Minnesota

21. Hennepin County Medical Center

\section{French ALS Consortium}

William Camu ${ }^{1}$, Kevin Mouzat ${ }^{2}$, Serge Lumbroso $^{2}$, Philippe Corcia $^{3}$, Vincent Meininger ${ }^{3}$, Gérard Besson ${ }^{4}$, Emmeline Lagrange ${ }^{5}$, Pierre Clavelou ${ }^{6}$, Nathalie Guy ${ }^{6}$, Philippe Couratier $^{7}$, Patrick Vourch ${ }^{8}$, Véronique Danel ${ }^{9}$, Emilien Bernard $^{10}$, Gwendal Lemasson ${ }^{11}$ 
1. ALS center, CHU Gui de Chauliac, University of Montpellier, Montpellier, France

2. Service de biochimie, CHU de Nîmes, Nîmes, France

3. ALS Center, CHU Bretonneau, Tours university, Tours, France

4. Service de Neurologie, Hôpital Michalon, La Tronche, France

5. Service d'explorations du système nerveux, Hôpital Michalon, La Tronche, France

6. ALS center, CHU Gabriel Montpied, Clermont-Ferrand, France

7. ALS center, CHU Dupuytren, Limoges, France

8. Département de Biochimie, CHU Bretonneau, Tours, France

9. ALS center, CHRU, Lille, France,

10. ALS center, CHU Wertheimer, Lyon, France

11. ALS center, CHU Pellegrin, Bordeaux, France

\section{Genomic Translation for ALS Care (GTAC) Consortium}

Matthew B. Harms ${ }^{1}$, David B. Goldstein ${ }^{2}$, Neil A. Shneider ${ }^{3}$, Stephen Goutman ${ }^{4}$, Zachary Simmons ${ }^{5}$, Timothy M. Miller ${ }^{6}$, Siddharthan Chandran ${ }^{7}$, Suvankar Pal ${ }^{7}$, George Manousakis $^{8}$, Stanley H. Appel ${ }^{9}$, Ericka Simpson ${ }^{9}$, Leo Wang ${ }^{10}$, Robert H Baloh ${ }^{11}$, Summer Gibson $^{12}$, Richard Bedlack ${ }^{13}$, David Lacomis ${ }^{14}$, Dhruv Sareen ${ }^{15}$, Alexander Sherman $^{16}$, Lucie Bruijn ${ }^{17}$, Michelle Penny ${ }^{18}$

1. Department of Neurology/Institute of Genomic Medicine/Elanor and Lou Gehrig ALS Center, Columbia University, New York, New York 10032, USA

2. Institute for Genomic Medicine, Columbia University, New York, NY 10032, USA

3. Department of Neurology/Elanor and Lou Gehrig ALS Center, Columbia University, New York, NY 10032, USA

4. Department of Neurology, University of Michigan, Ann Arbor, Michigan 48109, USA

5. Department of Neurology, The Pennsylvania State University College of Medicine, Hershey, Hershey, PA 17033, USA

6. Department of Neurology, Washington University in St. Louis, St. Louis, MO, USA

7. Centre for Clinical Brain Sciences, University of Edinburgh, Edinburgh, Scotland, UK

8. Department of Neurology, University of Minnesota, Minneapolis, MN, USA

9. Stanley Appel Department of Neurology, Houston Methodist, Houston, TX, USA

10. Department of Neurology, University of Washington, Seattle, WA, USA

11. Department of Neurology, Cedars Sinai Medical Center, Los Angeles, CA, USA

12. Department of Neurology, University of Utah, Salt Lake City, UT, USA

13. Duke ALS Clinic and Durham VA Medical Center, Duke University School of Medicine, Durham, NC 27708, USA

14. Department of Neurology and Pathology, University of Pittsburgh School of Medicine, Pittsburgh, PA, USA

15. Department of Biomedical Sciences, Cedars-Sinai Medical Center, Los Angeles, CA, USA

16. Neurological Clinical Research Institute, Massachusetts General Hospital, Boston, MA, USA 
17. Amyotrophic Lateral Sclerosis Association, , Washington DC, , USA

18. Computational Biology, Biogen, Cambridge, MA, USA

\section{ITALSGEN Consortium}

Francesco O. Logullo ${ }^{1}$, Isabella Simone ${ }^{2}$, Giancarlo Logroscino ${ }^{2,3}$, Fabrizio Salvi ${ }^{4}$, Ilaria Bartolomei $^{4}$, Giuseppe Borghero ${ }^{5}$, Maria Rita Murru ${ }^{5}$, Emanuela Costantino ${ }^{5}$, Carla Pani ${ }^{5}$, Roberta Puddu ${ }^{5}$, Carla Caredda ${ }^{5}$, Valeria Piras ${ }^{5}$, Stefania Tranquilli ${ }^{5}$, Stefania $\mathrm{Cuccu}^{5}$, Daniela Corongiu ${ }^{5}$, Maurizio Melis ${ }^{5}$, Antonio Milia ${ }^{5}$, Francesco Marrosu ${ }^{5}$, Maria Giovanna Marrosu ${ }^{5}$, Gianluca Floris ${ }^{5}$, Antonino Cannas ${ }^{5}$, Stefania Tranquilli ${ }^{5}$, Margherita Capasso $^{6}$, Claudia Caponnetto ${ }^{7}$, Gianluigi Mancardi ${ }^{7}$, Paola Origone ${ }^{7}$, Paola Mandich ${ }^{7}$, Francesca L. Conforti ${ }^{8}$, Sebastiano Cavallaro ${ }^{8}$, Gabriele Mora ${ }^{9}$, Kalliopi Marinou ${ }^{9}$, Riccardo Sideri ${ }^{9}$, Silvana Penco ${ }^{10,33}$, Lorena Mosca ${ }^{10}$, Christian Lunetta ${ }^{11}$, Giuseppe Lauria Pinter ${ }^{12}$, Massimo Corbo ${ }^{13}$, Nilo Riva ${ }^{14}$, Paola Carrera ${ }^{14}$, Paolo Volanti ${ }^{15}$, Jessica Mandrioli $^{16}$, Nicola Fini ${ }^{16}$, Antonio Fasano ${ }^{16}$, Lucio Tremolizzo ${ }^{17}$, Alessandro Arosio ${ }^{17}$, Carlo Ferrarese ${ }^{17}$, Francesca Trojsi ${ }^{18}$, Gioacchino Tedeschi ${ }^{19}$, Maria Rosaria Monsurrò ${ }^{19}$, Giovanni Piccirillo ${ }^{19}$, Cinzia Femiano ${ }^{19}$, Anna Ticca ${ }^{20}$, Enzo Ortu ${ }^{21}$, Vincenzo La Bella ${ }^{22}$, Rossella Spataro $^{22}$, Tiziana Colletti ${ }^{22}$, Mario Sabatelli ${ }^{23}$, Marcella Zollino ${ }^{23}$, Amelia Conte $^{23}$, Marco Luigetti ${ }^{23}$, Serena Lattante ${ }^{23}$, Giuseppe Marangi ${ }^{23}$, Marialuisa Santarelli ${ }^{24}$, Antonio Petrucci ${ }^{25}$, Maura Pugliatti ${ }^{26}$, Angelo Pirisi ${ }^{26}$, Leslie D. Parish ${ }^{26}$, Patrizia Occhineri $^{26}$, Fabio Giannini ${ }^{27}$, Stefania Battistini ${ }^{27}$, Claudia Ricci ${ }^{27}$, Michele Benigni ${ }^{27}$, Tea B. $\mathrm{Cau}^{28}$, Daniela Loi ${ }^{28}$, Andrea Calvo ${ }^{29}$, Cristina Moglia ${ }^{29}$, Maura Brunetti ${ }^{30}$, Marco Barberis $^{29}$, Gabriella Restagno ${ }^{30}$, Federico Casale ${ }^{29}$, Giuseppe Marrali ${ }^{29}$, Giuseppe Fuda ${ }^{29}$, Irene Ossola ${ }^{29}$, Stefania Cammarosano ${ }^{29}$, Antonio Canosa ${ }^{29}$, Antonio Ilardi ${ }^{29}$, Umberto Manera $^{29}$, Maurizio Grassano ${ }^{29}$, Raffaella Tanel ${ }^{31}$, Fabrizio Pisano ${ }^{32}$

1. Neurological Clinic, Marche Polytechnic University, Ancona, Italy

2. Department of Basic Medical Sciences, Neurosciences and Sense Organs, University of Bari, Bari, Italy

3. Department of Clinical and Research Neurology, "Pia Fondazione Cardinal G. Panico" Hospital, Tricase (LE), Bari, Italy

4. Center for Diagnosis and Cure of Rare Diseases, Department of Neurology, IRCCS Institute of Neurological Sciences, Bologna, Italy

5. Department of Neurology, Azienda Universitario Ospedaliera di Cagliari and University of Cagliari, Cagliari, Italy

6. Department of Neurology, University of Chieti, Chieti, Italy

7. Department of Neurosciences, Ophthalmology, Genetics, Rehabilitation, Maternal and Child Health, Ospedale Policlinico San Martino, Genoa, Italy

8. Institute of Neurological Sciences, National Research Council, Mangone, Cosenza, Italy

9. Department of Neurological Rehabilitation, Fondazione Salvatore Maugeri, IRCCS, Istituto Scientifico di Milano, Milano, Italy

10. Department of Laboratory Medicine, Medical Genetics, Niguarda Ca' Granda Hospital, Milan, Italy

11. NeuroMuscular Omnicenter, Serena Onlus Foundation, Milan, Italy 
12. Neuroalgology and Headache Unit, IRCCS Fondazione Istituto Neurologico "Carlo Besta", Milano, Italy

13. Department of Neurorehabilitation Sciences (P.T., M.C.), Casa Cura Policlinico, Milan, Italy

14. Department of Neurology and Institute of Experimental Neurology (INSPE), IRCCS San Raffaele Scientific Institute, Milan, Italy

15. Neurorehabilitation Unit/ALS Center, Scientific Clinical Institutes (ICS) Maugeri, IRCCS, Mistretta, Messina, Italy

16. Department of Neuroscience, S. Agostino-Estense Hospital, University of Modena and Reggio Emilia, Modena, Italy

17. Neurology Unit, School of Medicine and Surgery and NeuroMI, University of Milano-Bicocca, Monza, Italy

18. Department of Medical, Surgical, Neurological, Metabolic and Aging Sciences, University of Campania "Luigi Vanvitelli", Naples, Italy

19. Department of Medical, Surgical Neurological Metabolic and Aging Sciences, Second University of Naples, Naples, Italy

20. Department of Neurology, Azienda Ospedaliera San Francesco, Nuoro, Italy.

21. Division of Neurology, "A. Segni" Hospital, Ozieri, Italy

22. ALS Clinical Research Center, Bio. Ne. C., University of Palermo, Palermo, Italy.

23. Centro Clinico NEMO-Roma. Neurological Institute, Catholic University and I.C.O.M.M. Association for ALS Research, Rome, Italy

24. Department of Medicine, Azienda Complesso Ospedaliero, San Filippo Neri, Rome, Italy

25. Neurology Department, San Camillo Hospital, Rome, Italy

26. Department of Biomedical and Surgical Sciences, Section of Neurological, Psychiatric and Psychological Sciences, University of Ferrara, Ferrara, Italy

27. Department of Medical, Surgical and Neurological Sciences, University of Siena, Siena, Italy

28. Azienda Sanitaria Locale n. 2, Olbia-Tempio, Olbia, Italy

29. Rita Levi Montalcini Department of Neuroscience, Amyotrophic Lateral Sclerosis Center, University of Turin, Turin, Italy; Azienda Ospedaliero Universitaria Città della Salute e della Scienza, Turin, Italy

30. Molecular Genetics Unit, Department of Clinical Pathology, A.S.O. O.I.R.M.-S. Anna, 10126 Turin, Italy

31. Department of Neurology, Santa Chiara Hospital, Trento, Italy

32. Department of Neurological Rehabilitation, "Salvatore Maugeri" Clinical-Scientific Institute, Istituto di Ricovero e Cura a Carattere Scientifico, Veruno, Italy

33. Deceased

\section{NYGC ALS Consortium}

Hemali Phatnani ${ }^{1}$, Justin Kwan ${ }^{2}$, Dhruv Sareen ${ }^{3}$, James R. Broach ${ }^{4}$, Zachary Simmons ${ }^{5}$, Ximena Arcila-Londono ${ }^{6}$, Edward B. Lee ${ }^{7}$, Vivianna M. Van Deerlin ${ }^{7}$, Neil A. Shneider ${ }^{8}$, Ernest Fraenkel ${ }^{9}$, Lyle W. Ostrow ${ }^{10}$, Frank Baas ${ }^{11}$, Noah Zaitlen ${ }^{12}$, James D. Berry ${ }^{13}$, Andrea Malaspina ${ }^{14}$, Pietro Fratta ${ }^{15}$, Gregory A. Cox ${ }^{16}$, Leslie M. Thompson ${ }^{17}$, Steve Finkbeiner $^{18}$, Efthimios Dardiotis ${ }^{19}$, Timothy M. Miller ${ }^{20}$, Siddharthan Chandran ${ }^{21}$, 
Suvankar Pal ${ }^{21}$, Eran Hornstein ${ }^{22}$, Daniel J. MacGowan ${ }^{23}$, Terry Heiman-Patterson ${ }^{24}$, Molly G. Hammell ${ }^{25}$, Nikolaos. A. Patsopoulos ${ }^{26,27}$, Joshua Dubnau ${ }^{28}$, Avindra Nath ${ }^{29}$

1. Center for Genomics of Neurodegenerative Diseases (CGND), New York Genome Center, New York, NY

2. Department of Neurology, University of Maryland School of Medicine, University of Maryland ALS Clinic, Baltimore, MD"

3. Cedars-Sinai Department of Biomedical Sciences, Board of Governors Regenerative Medicine Institute and Brain Program, Cedars-Sinai Medical Center, and Department of Medicine, University of California, Los Angeles, CA

4. Department of Biochemistry and Molecular Biology, Penn State Institute for Personalized Medicine, The Pennsylvania State University, Hershey, PA

5. Department of Neurology, The Pennsylvania State University, Hershey, PA

6. Department of Neurology, Henry Ford Health System, Detroit, MI

7. Department of Pathology and Laboratory Medicine, Perelman School of Medicine, University of Pennsylvania, Philadelphia, PA

8. Department of Neurology, Center for Motor Neuron Biology and Disease, Institute for Genomic Medicine, Columbia University, New York, NY

9. Department of Biological Engineering, Massachusetts Institute of Technology, Cambridge, MA

10. Department of Neurology, Johns Hopkins School of Medicine, Baltimore, MD

11. Department of Neurogenetics, Academic Medical Centre, Amsterdam and Leiden University Medical Center, Leiden, The Netherlands

12. Department of Medicine, Lung Biology Center, University of California, San Francisco, CA

13. ALS Multidisciplinary Clinic, Neuromuscular Division, Department of Neurology, Harvard Medical School, and Neurological Clinical Research Institute, Massachusetts General Hospital, Boston, MA

14. Centre for Neuroscience and Trauma, Blizard Institute, Barts and The London School of Medicine and Dentistry, Queen Mary University of London, London, and Department of Neurology, Basildon University Hospital, Basildon, United Kingdom

15. Institute of Neurology, National Hospital for Neurology and Neurosurgery, University College London, London, United Kingdom

16. The Jackson Laboratory, Bar Harbor, ME

17. Department of Psychiatry \& Human Behavior, Department of Biological Chemistry, School of Medicine, and Department of Neurobiology and Behavior, School of Biological Sciences, University California, Irvine, CA

18. Taube/Koret Center for Neurodegenerative Disease Research, Roddenberry Center for Stem Cell Biology and Medicine, Gladstone Institute

19. Department of Neurology \& Sensory Organs, University of Thessaly, Thessaly, Greece

20. Department of Neurology,Washington University in St. Louis, St. Louis, MO

21. Centre for Clinical Brain Sciences, Anne Rowling Regenerative Neurology Clinic, Euan MacDonald Centre for Motor Neurone Disease Research, University of Edinburgh, Edinburgh, United Kingdom

22. Department of Molecular Genetics, Weizmann Institute of Science, Rehovot, Israel 
23. Department of Neurology, Icahn School of Medicine at Mount Sinai, New York, NY

24. Center for Neurodegenerative Disorders, Department of Neurology, the Lewis Katz School of Medicine, Temple University, Philadelphia, PA

25. Cold Spring Harbor Laboratory, Cold Spring Harbor, NY

26. Computer Science and Systems Biology Program, Ann Romney Center for Neurological Diseases, Department of Neurology and Division of Genetics in Department of Medicine, Brigham and Women's Hospital, Boston, MA

27. Program in Medical and Population Genetics, Broad Institute, Cambridge, MA

28. Department of Anesthesiology, Stony Brook University, Stony Brook, NY

29. Section of Infections of the Nervous System, National Institute of Neurological Disorders and Stroke, NIH, Bethesda, MD

\section{Project MinE ALS Sequencing Consortium}

Ahmad Al Kheifat ${ }^{1}$, Ammar Al-Chalabi ${ }^{1}$, Peter Andersen ${ }^{2}$, A. Nazli Basak ${ }^{3}$, Ian P Blair ${ }^{4}$, Adriano Chio ${ }^{5}$, Jonathan Cooper-Knock ${ }^{6}$, Philippe Corcia ${ }^{7}$, Philippe Couratier ${ }^{8}$, Mamede de Carvalho ${ }^{9}$, Annelot Dekker ${ }^{10}$, Vivian Drory ${ }^{11}$, Alberto Garcia Redondo ${ }^{12}$, Marc Gotkine $^{13}$, Orla Hardiman ${ }^{14,15}$, Winston Hide ${ }^{6,16}$, Alfredo Iacoangeli ${ }^{17}$, Glass Jonathan ${ }^{18}$, Kevin Kenna ${ }^{19}$, Matthew Kiernan ${ }^{20}$, Maarten Kooyman ${ }^{21}$, John Landers ${ }^{19}$, Russell McLaughlin $^{22}$, Bas Middelkoop ${ }^{10}$, Jonathan Mill' ${ }^{23,1}$, Miguel Mitne Neto ${ }^{24}$, Mattieu Moisse $^{25,26,27}$, Jesus Mora Pardina ${ }^{28}$, Karen Morrison ${ }^{29}$, Stephen Newhouse ${ }^{17,30}$, Susana Pinto $^{9}$, Sara Pulit ${ }^{10}$, Wim Robberecht ${ }^{25,26,27}$, Aleksey Shatunov ${ }^{1}$, Pamela Shaw ${ }^{6}$, Chris Shaw $^{1}$, Vincenzo Silani ${ }^{31}$, William Sproviero ${ }^{1}$, Gijs Tazelaar ${ }^{10}$, Nicola Ticozzi ${ }^{31}$, Philip van Damme ${ }^{25,26,27}$, Leonard van den Berg ${ }^{10}$, Rick van der Spek ${ }^{10}$, Kristel van Eijk ${ }^{10}$, Michael van $\mathrm{Es}^{10}$, Wouter van Rheenen ${ }^{10}$, Joke van Vugt ${ }^{10}$, Jan Veldink ${ }^{10}$, Markus Weber $^{32}$, Kelly L Williams ${ }^{33}$, Mayana Zatz ${ }^{24}$, Denis C. Bauer ${ }^{25}$, Natalie A. Twine ${ }^{25}$

1. Maurice Wohl Clinical Neuroscience Institute, King's College London, Department of Basic and Clinical Neuroscience, London, UK.

2. Umeå University, Umeå, Sweden

3. Suna and Inan Kırac Foundation, Neurodegeneration Research Laboratory, Bogazici University, Istanbul, Turkey

4. Centre for MND Research, Faculty of Medicine and Health Sciences, Macquarie University, Sydney, NSW 2109, Australia

5. Universita degli Studi dei Torino, Turin, Italy

6. Sheffield Institute for Translational Neuroscience (SITraN), University of Sheffield, Sheffield, UK

7. Université François-Rabelais, Tours

8. Université de Limoges, France

9. Instituto de Medicina Molecular, University of Lisbon

10. Department of Neurology, Brain Center Rudolf Magnus, University Medical Center Utrecht, Utrecht, The Netherlands

11. Tel-Aviv Medical Center, Tel-Aviv, Israel

12. Hospital Carlos III, Madrid, Spain

13. Hadassah University Hospital, Jerusalem, Israel 
14. Academic Unit of Neurology, Trinity College Dublin, Trinity Biomedical Sciences Institute, Dublin, Republic of Ireland

15. Department of Neurology, Beaumont Hospital, Dublin, Republic of Ireland.

16. Biostatistics Department, Harvard School of Public Health, Boston, Massachusetts, USA.

17. Department of Biostatistics, IoPPN, King's College London, London, US.

18. Emory University, Atlanta, USA

19. Department of Neurology, University of Massachusetts Medical School, Worcester, MA, USA

20. Macquarie University, Sydney, Australia

21. SURFsara, Amsterdam, the Netherlands.

22. Population Genetics Laboratory, Smurfit Institute of Genetics, Trinity College Dublin, Dublin, Republic of Ireland.

23. University of Exeter Medical School, Exeter University, St Luke's Campus, Magdalen Street, Exeter EX1 2LU, UK

24. Universidade de São Paulo, Brazil

25. KU Leuven - University of Leuven, Department of Neurosciences, Experimental Neurology and Leuven Research Institute for Neuroscience and Disease (LIND), B3000 Leuven, Belgium

26. VIB, Vesalius Research Center, Laboratory of Neurobiology, Leuven, Belgium

27. University Hospitals Leuven, Department of Neurology, Leuven, Belgium

28. Hospital San Rafael, Madrid, Spain

29. Faculty of Medicine, University of Southampton, Southampton, UK

30. Biomedical Research Centre for Mental Health, IoPPN, King's College London, London, UK

31. Department of Neurology and Laboratory of Neuroscience, IRCCS Instituto Auxologico Italiano, University of Milan, Milan, Italy

32. Neuromuscular Disease Center/ALS Clinic, Kantonsspital St. Gallen, St. Gallen, Switzerland

33. Faculty of Medicine and Health Sciences, Macquarie University, Sydney, NSW 2109, Australia

34. Commonwealth Scientific and Industrial Research Organization, Sydney, New South Wales, Australia

\section{SLAGEN Consortium}

Vincenzo Silani ${ }^{1,2}$, Nicola Ticozzi ${ }^{1,2}$, Cinzia Gellera ${ }^{3}$, Antonia Ratti $^{1,2}$, Franco Taroni ${ }^{3}$, Giuseppe Lauria ${ }^{4}$, Federico Verde ${ }^{1}$, Isabella Fogh ${ }^{1,5}$, Cinzia Tiloca ${ }^{1}$, Giacomo P. Comi ${ }^{2,6}$, Gianni Sorarù ${ }^{7}$, Cristina Cereda $^{8}$, Sandra D'Alfonso ${ }^{9}$, Lucia Corrado ${ }^{9}$, Fabiola De Marchi $^{10}$, Stefania Corti ${ }^{2,6}$, Mauro Ceroni ${ }^{8,11}$, Letizia Mazzini ${ }^{10}$, Gabriele Siciliano ${ }^{12}$, Massimiliano Filosto ${ }^{13}$, Maurizio Inghilleri ${ }^{14}$, Silvia Peverellii ${ }^{1}$, Claudia Colombrita ${ }^{12}$, Barbara Poletti ${ }^{1}$, Luca Maderna ${ }^{1}$, Roberto Del Bo ${ }^{2,6}$, Stella Gagliardi $^{8}$, Giorgia Querin ${ }^{7}$, Cinzia Bertolin ${ }^{7}$, Viviana Pensato ${ }^{3}$, Barbara Castellotti ${ }^{3}$

1. Department of Neurology, IRCCS Istituto Auxologico Italiano, Milan, Italy 
2. Department of Pathophysiology and Transplantation, 'Dino Ferrari' Center, Università degli Studi di Milano, Milan, Italy

3. Unit of Genetics of Neurodegenerative and Metabolic Diseases, Fondazione IRCCS Istituto Neurologico 'Carlo Besta', Milan, Italy.

4. 3rd Neurology Unit, Motor Neuron Diseases Center, Fondazione IRCCS Istituto Neurologico 'Carlo Besta', Milan, Italy.

5. Maurice Wohl Clinical Neuroscience Institute, King's College London, Department of Basic and Clinical Neuroscience, Institute of Psychiatry, Psychology and Neuroscience, London, UK.

6. Neurology Unit, IRCCS Foundation Ca' Granda Ospedale Maggiore Policlinico, Milan, Italy.

7. Department of Neurosciences, University of Padova, Padova, Italy

8. Experimental Neurobiology Laboratory, 'C. Mondino' National Institute of Neurology Foundation, IRCCS, Pavia, Italy

9. Department of Health Sciences, University of Eastern Piedmont, Novara, Italy

10. Department of Neurology, Azienda Ospedaliera Universitaria Maggiore della Carità, Novara, Italy

11. Department of Neurological Sciences, University of Pavia, Pavia, Italy

12. Neurology Unit, Department of Clinical and Experimental Medicine, University of Pisa, Pisa, Italy

13. Center for Neuromuscular Diseases and Neuropathies, Unit of Neurology ASST "Spedali Civili", University of Brescia, Brescia, Italy

14. Rare Neuromuscular Diseases Centre, Department of Neurology and Psychiatry, Sapienza University, Rome, Italy 